\title{
Catalytic Site-Selective Carbamoylation of Pyranosides
}

\author{
Jérôme Alsarraf,* Lucas Petitpoisson and André Pichette
}

Centre de recherche sur la boréalie (CREB), Laboratoire d'analyse et de séparation des essences végétales (LASEVE), Université du Québec à Chicoutimi, 555 boulevard de l'Université, Chicoutimi G7H 2B1, Québec, Canada.

*Corresponding author: jerome1_alsarraf@uqac.ca, +1 4185455011 ext 2273

\section{Table of contents}

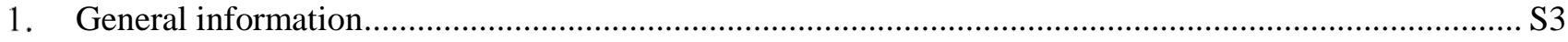

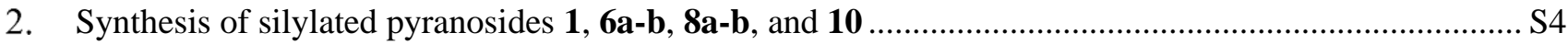

a. $\quad$ Methyl 6-O-(tert-butyldimethyl)silyl- $\alpha$-D-mannopyranoside (1) ..................................................... S4

b. Methyl 6-O-(tert-butyldimethyl)silyl- $\beta$-D-galactopyranoside (6a) .............................................. S4

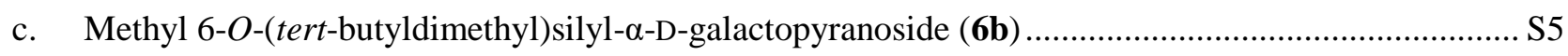

d. 4-Methoxyphenyl 6-O-(tert-butyldimethyl)silyl- $\alpha$-D-mannopyranoside (8a) .................................. S5

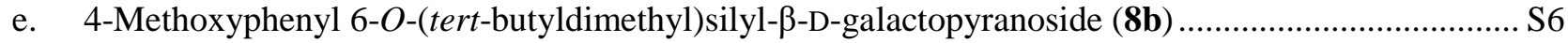

f. $\quad$ Methyl 6-O-(tert-butyldimethyl)silyl- $\alpha$-D-glucopyranoside (10) .................................................... S6

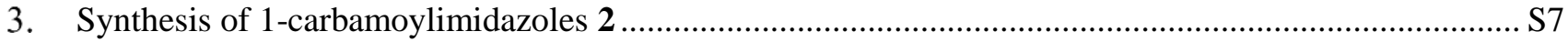

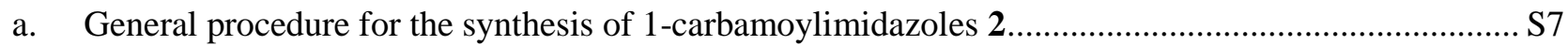

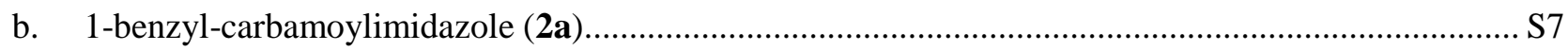

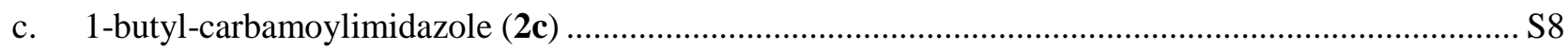

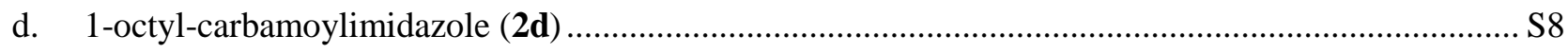

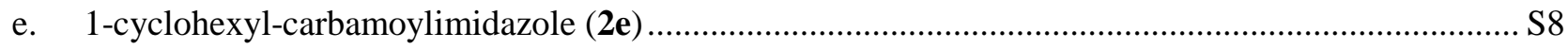

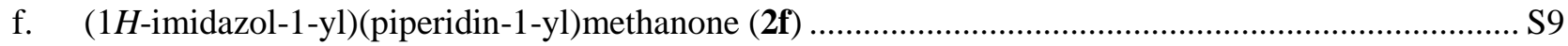

4. Synthesis of carbamate-bearing pyranosides 3a-e, 7a-b, and 9a-e …................................................ 9

a. General procedure for the site-selective carbamoylation of pyranosides ......................................... S9

b. Methyl 3-O-benzylcarbamoyl-6-O-(tert-butyldimethyl)silyl- $\alpha$-D-mannopyranoside (3a)................... S9

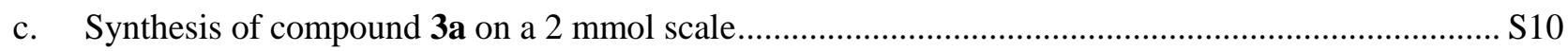

d. Methyl 3-O-methylcarbamoyl-6-O-(tert-butyldimethyl)silyl- $\alpha$-D-mannopyranoside (3b) ................. S10

e. Methyl 3-O-butylcarbamoyl-6-O-(tert-butyldimethyl)silyl- $\alpha$-D-mannopyranoside (3c) ................... S10

f. $\quad$ Methyl 3-O-octylcarbamoyl-6- $O$-(tert-butyldimethyl)silyl- $\alpha$-D-mannopyranoside (3d) ................... S11

g. $\quad$ Methyl 3-O-cyclohexylcarbamoyl-6-O-(tert-butyldimethyl)silyl- $\alpha$-D-mannopyranoside (3e) ........... S11

h. Methyl 3-O-benzylcarbamoyl-6-O-(tert-butyldimethyl)silyl- $\beta$-D-galactopyranoside (7a) ................. S12

i. $\quad$ Methyl 3-O-benzylcarbamoyl-6-O-(tert-butyldimethyl)silyl- $\alpha$-D-galactopyranoside (7b) ................ S12 
j. $\quad$ 4-Methoxyphenyl 3-O-benzylcarbamoyl-6-O-(tert-butyldimethyl)silyl- $\alpha$-D-mannopyranoside (9a). S13

k. 4-Methoxyphenyl 3-O-benzylcarbamoyl-6-O-(tert-butyldimethyl)silyl- $\beta$-D-galactopyranoside (9b) S13

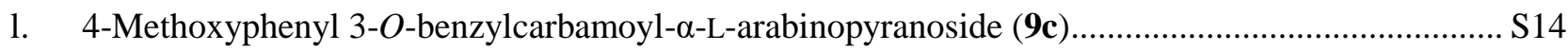

m. 4-Methoxyphenyl 3-O-benzylcarbamoyl- $\alpha$-L-lyxopyranoside (9d) .............................................. S14

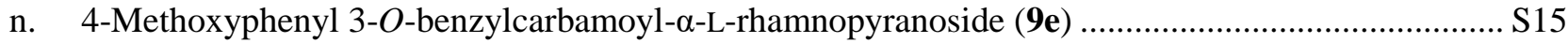

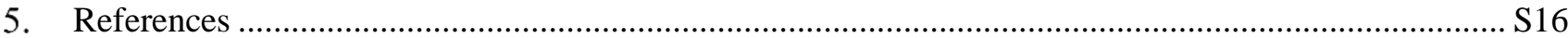

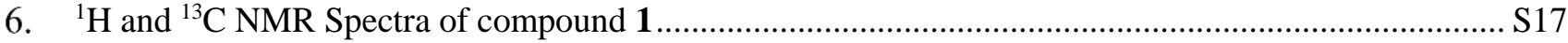

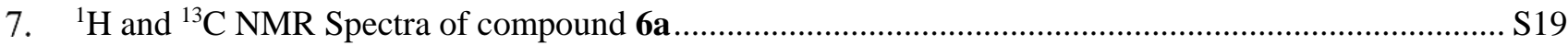

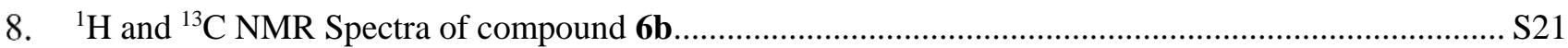

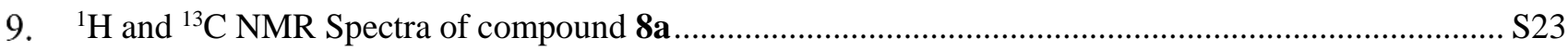

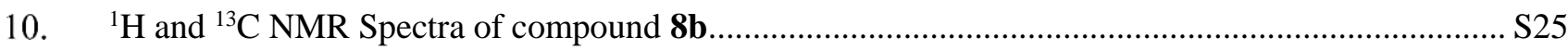

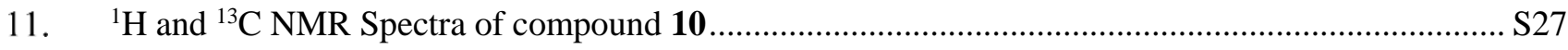

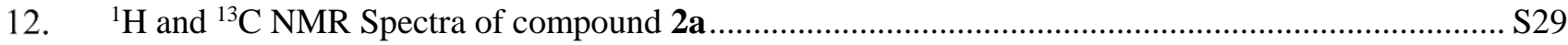

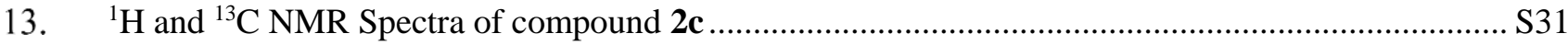

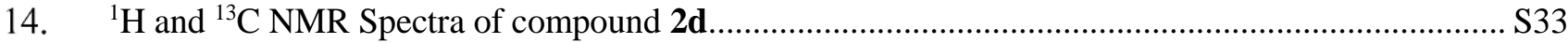

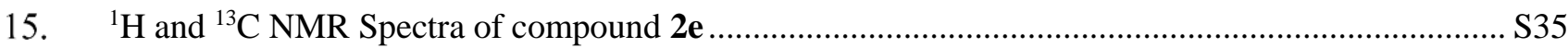

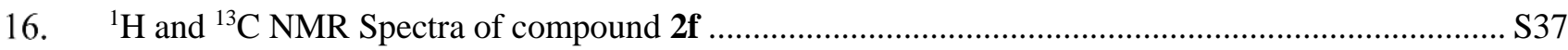

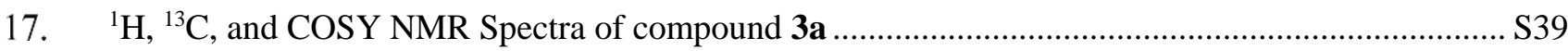

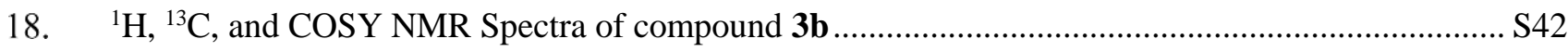

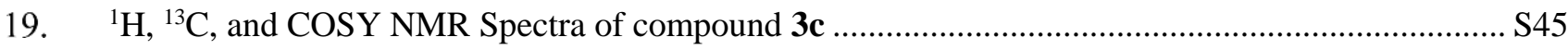

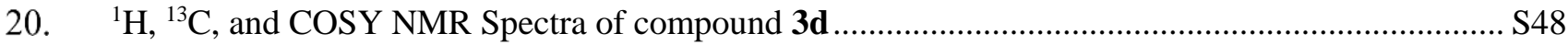

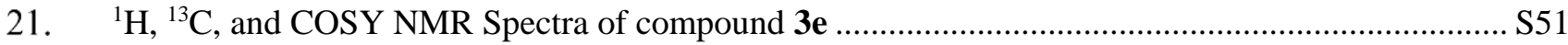

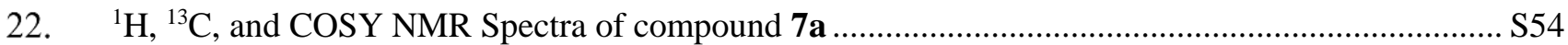

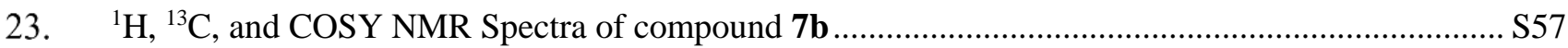

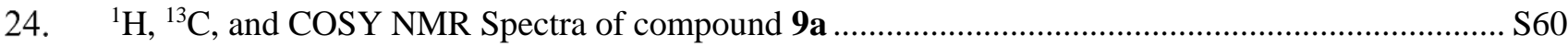

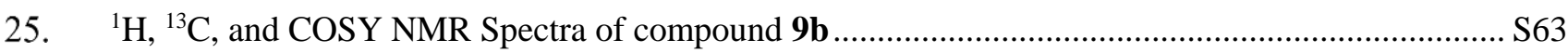

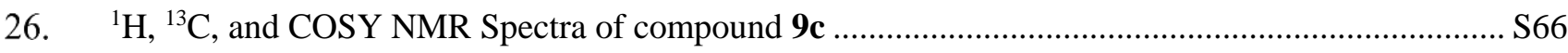

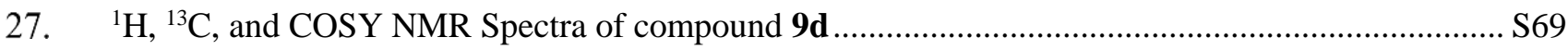

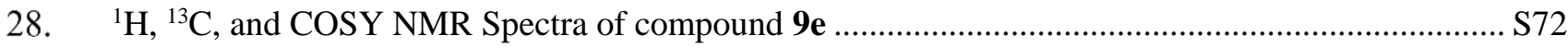




\section{General information}

Unless otherwise noted, all starting materials and solvents were purchased from commercial sources and used as received without further purification. Reactions were conducted under argon atmosphere, using anhydrous solvent, unless otherwise noted. Reactions that required heating were performed using Radleys Heat-on block systems. All reaction where monitored by thin-layer chromatography (TLC) using normal phase silica gel $60 \mathrm{~F}_{254} 0.25 \mathrm{~mm}$ precoated aluminum foil plates. TLC were visualized under UV $(254 \mathrm{~nm})$ or revealed using ceric ammonium molybdate. Flash chromatographic purifications were performed using normal phase silica gel $60(15-40 \mu \mathrm{m})$. NMR spectra were recorded with a Bruker Avance 400 spectrometer at $400 \mathrm{MHz}$ for ${ }^{1} \mathrm{H}$ nuclei and $101 \mathrm{MHz}$ for ${ }^{13} \mathrm{C}$ nuclei, using deuterated chloroform, methanol, or dimethyl sulfoxide as the solvent. Chemical shifts $\delta$ were reported in ppm relative to the solvent residual peak ${ }^{1}\left(\mathrm{CDCl}_{3} \delta_{\mathrm{H} / \mathrm{C}} 7.26 / 77.16 \mathrm{ppm} ; \mathrm{CD}_{3} \mathrm{OD} \delta_{\mathrm{H} / \mathrm{C}} 3.31 / 49.00 \mathrm{ppm}\right.$; $\left.\left(\mathrm{CD}_{3}\right)_{2} \mathrm{SO} \delta_{\mathrm{H} / \mathrm{C}} 2.50 / 39.52\right)$ and coupling constants $J$ in Hertz $(\mathrm{Hz})$. Multiplicities were reported using the following abbreviations: s, singlet; $d$, doublet, t, triplet; q, quartet; $m$, multiplet; br, broad. HRMS spectra were recorded on an Agilent 6224 MS-TOF mass spectrometer equipped with an electrospray source. 


\section{Synthesis of silylated pyranosides $\mathbf{1}, \mathbf{6} \boldsymbol{a}-\boldsymbol{b}, \mathbf{8} \boldsymbol{a}-\boldsymbol{b}$, and $\mathbf{1 0}$}

a. Methyl 6-O-(tert-butyldimethyl)silyl- $\alpha$-D-mannopyranoside (1)

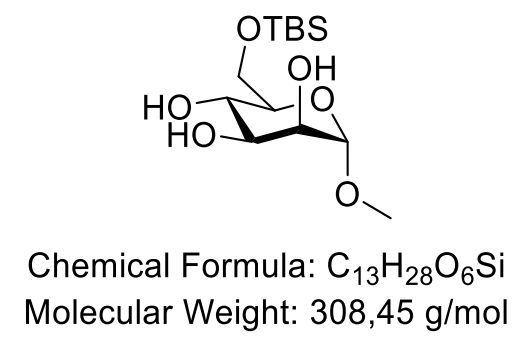

A mixture of methyl $\alpha$-D-mannopyranoside $(1.00 \mathrm{~g}, 5.15 \mathrm{mmol})$ and imidazole $(0.701 \mathrm{~g}, 10.3 \mathrm{mmol})$ in DMF $(8.0 \mathrm{~mL})$ was stirred $15 \mathrm{~min}$ at $0{ }^{\circ} \mathrm{C}$. tert-Butyldimethylsilyl chloride $(0.913 \mathrm{~g}, 6.18 \mathrm{mmol})$ was added at $0^{\circ} \mathrm{C}$. Then, the mixture was stirred $30 \mathrm{~min}$ at room temperature and controlled by TLC. The mixture was diluted with ethyl acetate $(50 \mathrm{~mL})$, washed with a saturated aqueous solution of $\mathrm{NaHCO}_{3}(2$ $\times 25 \mathrm{~mL})$ and a saturated aqueous solution of $\mathrm{NaCl}(2 \times 25 \mathrm{~mL})$. The organic layer was dried over $\mathrm{Na}_{2} \mathrm{SO}_{4}$, filtered and the solvent rotary evaporated. The crude residue was chromatographed over silica gel using a gradient of ethyl acetate (from $0 \%$ to $50 \%$ ) in dichloromethane as the mobile phase to afford the title compound (1) as an off-white powder (1271.0 mg, 82\%). $R_{f}=0.35$ (dichloromethane/methanol 9:1); ${ }^{1} \mathrm{H}$ NMR $\left(\mathrm{CDCl}_{3}, 400 \mathrm{MHz}\right): \delta 4.71(\mathrm{~s}, 1 \mathrm{H}), 3.96-3.73(\mathrm{~m}, 5 \mathrm{H}), 3.63-3.51(\mathrm{~m}, 2 \mathrm{H}), 3.37(\mathrm{~s}, 3 \mathrm{H})$, 3.20 (br s, 1H), 2.85 (br s, 1H), 1.88 (br s, $1 \mathrm{H}), 0.90$ (s, 9H), 0.10 (s, 6H). $\left.{ }^{13} \mathrm{C} \mathrm{NMR} \mathrm{(CDCl} 3,101 \mathrm{MHz}\right)$ : $\delta 100.8,71.7,71.0,70.4,70.3,65.1,55.1,26.0,18.4,-5.3,-5.4$. NMR data were consistent with literature description. $^{2}$

b. Methyl 6-O-(tert-butyldimethyl)silyl- $\beta$-D-galactopyranoside (6a)

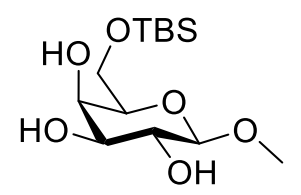

Chemical Formula: $\mathrm{C}_{13} \mathrm{H}_{28} \mathrm{O}_{6} \mathrm{Si}$

Molecular Weight: $308,45 \mathrm{~g} / \mathrm{mol}$

A mixture of methyl $\beta$-D-galactopyranoside $(500 \mathrm{mg}, 2.59 \mathrm{mmol})$ and imidazole $(353 \mathrm{mg}, 5.18 \mathrm{mmol})$ in DMF $(5.0 \mathrm{~mL})$ was stirred $15 \mathrm{~min}$ at $0^{\circ} \mathrm{C}$. tert-Butyldimethylsilyl chloride $(592 \mathrm{mg}, 3.93 \mathrm{mmol})$ was added at $0^{\circ} \mathrm{C}$. Then, the mixture was stirred $20 \mathrm{~h}$ at room temperature and controlled by TLC. The mixture was diluted with ethyl acetate $(25 \mathrm{~mL})$, washed with a saturated aqueous solution of $\mathrm{NaHCO}_{3}(2 \times 15$ $\mathrm{mL})$ and a saturated aqueous solution of $\mathrm{NaCl}(2 \times 15 \mathrm{~mL})$. The organic layer was dried over $\mathrm{Na}_{2} \mathrm{SO}_{4}$, filtered and the solvent rotary evaporated. The crude residue was chromatographed over silica gel using a gradient of ethyl acetate (from $0 \%$ to 50\%) in dichloromethane as the mobile phase to afford the title compound (6a) as an off-white powder (360.6 mg, 45\%). $R_{f}=0.34$ (dichloromethane/methanol 9:1); ${ }^{1} \mathrm{H}$ NMR $\left(\mathrm{CDCl}_{3}, 400 \mathrm{MHz}\right): \delta 4.16(\mathrm{~d}, 1 \mathrm{H}, J=7.6 \mathrm{~Hz}), 4.05-4.00(\mathrm{~m}, 1 \mathrm{H}), 3.91(\mathrm{dd}, 1 \mathrm{H}, J=10.4 \mathrm{~Hz}, J$ $=6.1 \mathrm{~Hz}), 3.85(\mathrm{dd}, 1 \mathrm{H}, J=10.5 \mathrm{~Hz}, J=5.2 \mathrm{~Hz}), 3.70-3.62(\mathrm{~m}, 1 \mathrm{H}), 3.61-3.56(\mathrm{~m}, 1 \mathrm{H}), 3.54(\mathrm{~s}, 3 \mathrm{H})$, 


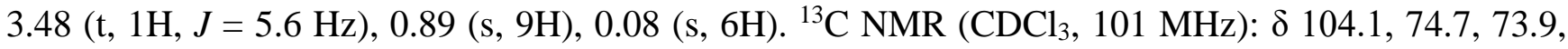
72.0, 69.0, 62.6, 57.1, 26.0, 18.4, -5.3. NMR data were consistent with literature description. ${ }^{3}$

c. Methyl 6-O-(tert-butyldimethyl)silyl- $\alpha$-D-galactopyranoside (6b)

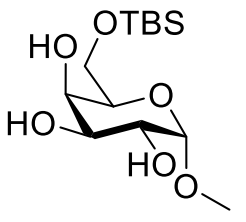

Chemical Formula: $\mathrm{C}_{13} \mathrm{H}_{28} \mathrm{O}_{6} \mathrm{Si}$

Molecular Weight: $308,45 \mathrm{~g} / \mathrm{mol}$

A mixture of methyl $\alpha$-D-galactopyranoside $(500 \mathrm{mg}, 2.59 \mathrm{mmol})$ and imidazole $(353 \mathrm{mg}, 5.18 \mathrm{mmol})$ in DMF $(5.0 \mathrm{~mL})$ was stirred $15 \mathrm{~min}$ at $0^{\circ} \mathrm{C}$. tert-Butyldimethylsilyl chloride $(592 \mathrm{mg}, 3.93 \mathrm{mmol})$ was added at $0^{\circ} \mathrm{C}$. Then, the mixture was stirred $20 \mathrm{~h}$ at room temperature and controlled by TLC. The mixture was diluted with ethyl acetate $(25 \mathrm{~mL})$, washed with a saturated aqueous solution of $\mathrm{NaHCO}_{3}(2 \times 15$ $\mathrm{mL})$ and a saturated aqueous solution of $\mathrm{NaCl}(2 \times 15 \mathrm{~mL})$. The organic layer was dried over $\mathrm{Na}_{2} \mathrm{SO}_{4}$, filtered and the solvent rotary evaporated. The crude residue was chromatographed over silica gel using a gradient of ethyl acetate (from $0 \%$ to $50 \%$ ) in dichloromethane as the mobile phase to afford the title compound (6b) as an off-white powder (270.6 mg, 34\%). $R_{f}=0.34$ (dichloromethane/methanol 9:1); ${ }^{1} \mathrm{H}$ NMR $\left(\mathrm{CDCl}_{3}, 400 \mathrm{MHz}\right): \delta 4.78(\mathrm{~d}, 1 \mathrm{H}, J=3.7 \mathrm{~Hz}), 4.12-4.00(\mathrm{~m}, 2 \mathrm{H}), 3.92-3.69(\mathrm{~m}, 5 \mathrm{H}), 3.66-$ $3.58(\mathrm{~m}, 1 \mathrm{H}), 0.89(\mathrm{~s}, 9 \mathrm{H}), 0.07(\mathrm{~s}, 6 \mathrm{H}) .{ }^{13} \mathrm{C} \mathrm{NMR}\left(\mathrm{CDCl}_{3}, 101 \mathrm{MHz}\right): \delta 99.8,71.3,70.2,69.8,69.6$, 63.2, 55.4, 26.0, 18.4, $-5.3,-5.3$. NMR data were consistent with literature description. ${ }^{2}$

d. 4-Methoxyphenyl 6-O-(tert-butyldimethyl)silyl- $\alpha$-D-mannopyranoside (8a)

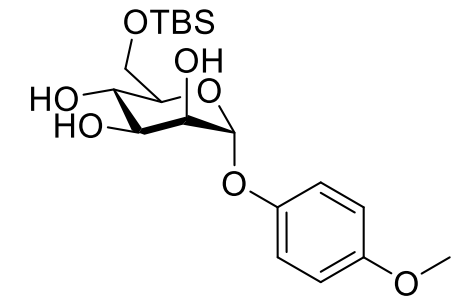

Chemical Formula: $\mathrm{C}_{19} \mathrm{H}_{32} \mathrm{O}_{7} \mathrm{Si}$

Molecular Weight: $400,54 \mathrm{~g} / \mathrm{mol}$

A mixture of 4-methoxyphenyl $\alpha$-D-mannopyranoside $(500 \mathrm{mg}, 1.747 \mathrm{mmol})$ and imidazole $(238 \mathrm{mg}$, $3.50 \mathrm{mmol})$ in DMF $(4.0 \mathrm{~mL})$ was stirred $15 \mathrm{~min}$ at $0^{\circ} \mathrm{C}$. tert-Butyldimethylsilyl chloride $(317 \mathrm{mg}, 2.10$ mmol) was added at $0^{\circ} \mathrm{C}$. Then, the mixture was stirred $30 \mathrm{~min}$ at room temperature and controlled by TLC. The mixture was then diluted with ethyl acetate $(25 \mathrm{~mL})$, washed with a saturated aqueous solution of $\mathrm{NaHCO}_{3}(2 \times 15 \mathrm{~mL})$ and a saturated aqueous solution of $\mathrm{NaCl}(2 \times 15 \mathrm{~mL})$. The organic layer was dried over $\mathrm{Na}_{2} \mathrm{SO}_{4}$, filtered and the solvent rotary evaporated. The crude residue was chromatographed over silica gel using a gradient of ethyl acetate (from $0 \%$ to $50 \%$ ) in dichloromethane as the mobile phase to afford the title compound (8a) as an off-white powder $(618.5 \mathrm{mg}, 88 \%) . \quad R_{f}=0.27$ (dichlormethane/ethyl acetate 1:1); $[\alpha]^{20}{ }_{\mathrm{D}}+92.0\left(c 0.1, \mathrm{CH}_{2} \mathrm{Cl}_{2}\right) ; \mathrm{HRMS}(\mathrm{ESI}) \mathrm{m} / \mathrm{z}$ calcd for $\mathrm{C}_{19} \mathrm{H}_{33} \mathrm{O}_{7} \mathrm{Si}$ 
$[\mathrm{M}+\mathrm{H}]^{+}$401.1990, found 401.1988; ${ }^{1} \mathrm{H} \mathrm{NMR}\left(\mathrm{CDCl}_{3}, 400 \mathrm{MHz}\right): \delta 6.99(\mathrm{~d}, 2 \mathrm{H}, J=9.1 \mathrm{~Hz}), 6.80(\mathrm{~d}$, $2 \mathrm{H}, J=9.1 \mathrm{~Hz}) 5.52-5.38(\mathrm{~m}, 1 \mathrm{H}), 4.19-4.10(\mathrm{~m}, 1 \mathrm{H}), 4.10-4.02(\mathrm{~m}, 1 \mathrm{H}), 3.95-3.68(\mathrm{~m}, 7 \mathrm{H}), 3.43$ (br s, 3H), $0.86(\mathrm{~s}, 9 \mathrm{H}), 0.06(\mathrm{~s}, 3 \mathrm{H}), 0.05$ (s, 3H). ${ }^{13} \mathrm{C} \mathrm{NMR}\left(\mathrm{CDCl}_{3}, 101 \mathrm{MHz}\right): \delta$ 155.1, 150.3, 118.0, $114.7,98.8,71.7,70.6,70.2,64.7,55.7,26.0,18.3,-5.3,-5.4$.

e. 4-Methoxyphenyl 6-O-(tert-butyldimethyl)silyl- $\beta$-D-galactopyranoside $(\mathbf{8 b})$

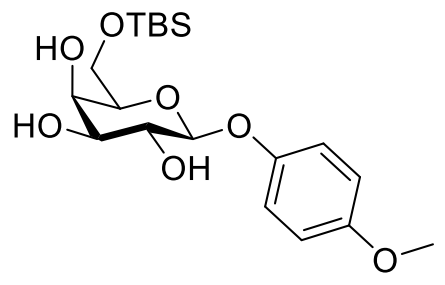

Chemical Formula: $\mathrm{C}_{19} \mathrm{H}_{32} \mathrm{O}_{7} \mathrm{Si}$

Molecular Weight: $400,54 \mathrm{~g} / \mathrm{mol}$

A mixture of 4-methoxyphenyl $\beta$-D-galactopyranoside $(500 \mathrm{mg}, 1.747 \mathrm{mmol})$ and imidazole $(238 \mathrm{mg}$, $3.50 \mathrm{mmol})$ in DMF $(4.0 \mathrm{~mL})$ was stirred $15 \mathrm{~min}$ at $0^{\circ} \mathrm{C}$. tert-Butyldimethylsilyl chloride $(317 \mathrm{mg}, 2.10$ mmol) was added at $0^{\circ} \mathrm{C}$. Then, the mixture was stirred 20 hours at room temperature and controlled by TLC. The mixture was then diluted with ethyl acetate $(25 \mathrm{~mL})$, washed with a saturated aqueous solution of $\mathrm{NaHCO}_{3}(2 \times 15 \mathrm{~mL})$ and a saturated aqueous solution of $\mathrm{NaCl}(2 \times 15 \mathrm{~mL})$. The organic layer was dried over $\mathrm{Na}_{2} \mathrm{SO}_{4}$, filtered and the solvent rotary evaporated. The crude residue was chromatographed over silica gel using a gradient of ethyl acetate (from $0 \%$ to $50 \%$ ) in dichloromethane as the mobile phase to afford the title compound $(\mathbf{8 b})$ as an off-white powder $(227.4 \mathrm{mg}, 33 \%) . R_{f}=0.26$ (dichloromethane/ethyl acetate 1:1); ${ }^{1} \mathrm{H} \mathrm{NMR}\left(\mathrm{CDCl}_{3}, 400 \mathrm{MHz}\right): \delta 6.99(\mathrm{~d}, 2 \mathrm{H}, J=8.5 \mathrm{~Hz}), 6.72(\mathrm{~d}$, $2 \mathrm{H}, J=8.5 \mathrm{~Hz}), 4.67$ (d, 1H, $J=7.4 \mathrm{~Hz}$ ), 4.55 (br s, 1H), 4.40 (br s, 1H), $4.09-3.95$ (m, 2H), $3.92-$ $3.64(\mathrm{~m}, 7 \mathrm{H}), 3.57-3.44(\mathrm{~m}, 1 \mathrm{H}), 0.86(\mathrm{~s}, 9 \mathrm{H}), 0.02(\mathrm{~s}, 3 \mathrm{H}), 0.01(\mathrm{~s}, 3 \mathrm{H}) .{ }^{13} \mathrm{C} \mathrm{NMR}\left(\mathrm{CDCl}_{3}, 101 \mathrm{MHz}\right)$ : $\delta 155.4,151.4,118.9,114.5,102.8,75.3,73.9,71.5,69.1,62.7,55.7,26.0,18.3,-5.3,-5.4$. NMR data were consistent with literature description. ${ }^{4}$

f. Methyl 6-O-(tert-butyldimethyl)silyl- $\alpha$-D-glucopyranoside (10)

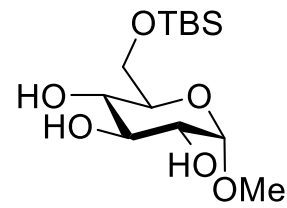

Chemical Formula: $\mathrm{C}_{13} \mathrm{H}_{28} \mathrm{O}_{6} \mathrm{Si}$

Molecular Weight: $308,45 \mathrm{~g} / \mathrm{mol}$

A mixture of methyl $\alpha$-D-glucopyranoside $(1.00 \mathrm{~g}, 5.15 \mathrm{mmol})$ and imidazole $(0.701 \mathrm{~g}, 10.3 \mathrm{mmol})$ in DMF $(8.0 \mathrm{~mL})$ was stirred $15 \mathrm{~min}$ at $0^{\circ} \mathrm{C}$. tert-Butyldimethylsilyl chloride $(0.913 \mathrm{~g}, 6.18 \mathrm{mmol})$ was added at $0^{\circ} \mathrm{C}$. Then, the mixture was stirred $30 \mathrm{~min}$ at room temperature and controlled by TLC. The mixture was diluted with ethyl acetate $(50 \mathrm{~mL})$, washed with a saturated aqueous solution of $\mathrm{NaHCO}_{3}(2$ $\times 25 \mathrm{~mL})$ and a saturated aqueous solution of $\mathrm{NaCl}(2 \times 25 \mathrm{~mL})$. The organic layer was dried over $\mathrm{Na}_{2} \mathrm{SO}_{4}$, filtered and the solvent rotary evaporated. The crude residue was chromatographed over silica 
gel using a gradient of ethyl acetate (from $0 \%$ to $50 \%$ ) in dichloromethane as the mobile phase to afford the title compound (10) as an off-white powder $(1157.1 \mathrm{mg}, 73 \%) . R_{f}=0.37$ (dichloromethane/methanol 9:1); ${ }^{1} \mathrm{H} \mathrm{NMR}\left(\mathrm{CDCl}_{3}, 400 \mathrm{MHz}\right): \delta 4.75(\mathrm{~d}, 1 \mathrm{H}, J=3.8 \mathrm{~Hz}), 3.89(\mathrm{dd}, 1 \mathrm{H}, J=10.5 \mathrm{~Hz}, J=4.7 \mathrm{~Hz})$, $3.81(\mathrm{dd}, 1 \mathrm{H}, J=10.5 \mathrm{~Hz}, J=5.1 \mathrm{~Hz}), 3.74(\mathrm{t}, 1 \mathrm{H}, J=9.1 \mathrm{~Hz}), 3.65-3.49(\mathrm{~m}, 3 \mathrm{H}), 3.42(\mathrm{~s}, 3 \mathrm{H}), 0.90$ $(\mathrm{s}, 9 \mathrm{H}), 0.10(\mathrm{~s}, 6 \mathrm{H}) .{ }^{13} \mathrm{C} \mathrm{NMR}\left(\mathrm{CDCl}_{3}, 101 \mathrm{MHz}\right): \delta 99.3,74.2,71.9,70.8,63.3,54.8,25.9,18.4,-5.2$. NMR data were consistent with literature description. ${ }^{3}$

\section{Synthesis of 1-carbamoylimidazoles 2}

a. General procedure for the synthesis of 1-carbamoylimidazoles 2
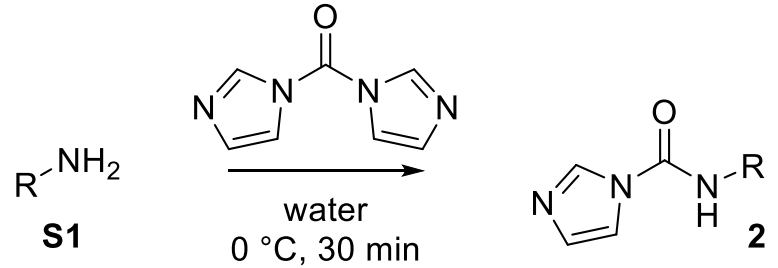

The amine $\mathbf{S 1}(5.00 \mathrm{mmol})$ was dissolved in water $(50 \mathrm{~mL})$ and stirred $15 \mathrm{~min}$ at $0^{\circ} \mathrm{C}$. Then $N, N^{\prime}-$ carbonyldiimidazole (CDI) $(6.00 \mathrm{mmol})$ was added, and the mixture was stirred for $30 \mathrm{~min}$ at $0^{\circ} \mathrm{C}$. The mixture was extracted with ethyl acetate $(2 \times 25 \mathrm{~mL})$, then the organic layers were pooled together and washed with a saturated aqueous solution of $\mathrm{NaHCO}_{3}(2 \times 25 \mathrm{~mL})$ and a saturated aqueous solution of $\mathrm{NaCl}(25 \mathrm{~mL})$. The organic layer was dried over $\mathrm{Na}_{2} \mathrm{SO}_{4}$, filtered and the solvent rotary evaporated. The crude residue was chromatographed over silica gel using a gradient of ethyl acetate (from $0 \%$ to $50 \%$ ) in dichloromethane as the mobile phase to afford the expected 1-carbamoylimidazole 2.

b. 1-benzyl-carbamoylimidazole (2a)

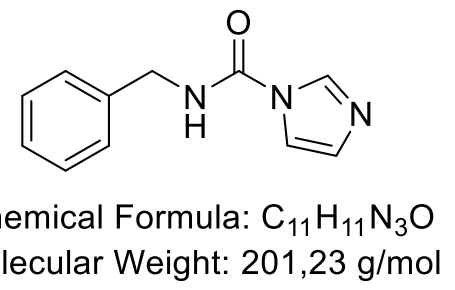

To a solution of benzylamine S1a $(2.14 \mathrm{~g}, 20 \mathrm{mmol})$ in water $(300 \mathrm{~mL})$ at $0{ }^{\circ} \mathrm{C}$ was added CDI $(3.89 \mathrm{~g}$, $24 \mathrm{mmol}$ ) and the mixture was stirred for $15 \mathrm{~min}$ at $0{ }^{\circ} \mathrm{C}$. The white precipitate was filtered and washed with cold water to afford the title compound $\mathbf{2 a}$ as a white powder $(3.837 \mathrm{~g}, 95 \%) . R_{f}=0.35$ (dichlormethane/ethyl acetate 1:1); ${ }^{1} \mathrm{H}$ NMR $\left(\left(\mathrm{CD}_{3}\right)_{2} \mathrm{SO}, 400 \mathrm{MHz}\right): \delta 9.10(\mathrm{~s}, 1 \mathrm{H}), 8.29(\mathrm{~s}, 1 \mathrm{H}), 7.72(\mathrm{~s}$, $1 \mathrm{H}), 7.43-7.20(\mathrm{~m}, 5 \mathrm{H}), 7.04(\mathrm{~s}, 1 \mathrm{H}), 4.47(\mathrm{~d}, 2 \mathrm{H}, J=5.4 \mathrm{~Hz}) .{ }^{13} \mathrm{C} \mathrm{NMR}\left(\left(\mathrm{CD}_{3}\right)_{2} \mathrm{SO}, 101 \mathrm{MHz}\right): \delta$ $149.0,138.5,136.1,129.7,128.5,127.4,127.2,116.6,43.5$. NMR data were consistent with literature description..$^{5}$ 
c. 1-butyl-carbamoylimidazole (2c)<smiles>CCCCNC(=O)n1ccnc1</smiles>

Chemical Formula: $\mathrm{C}_{8} \mathrm{H}_{13} \mathrm{~N}_{3} \mathrm{O}$

Molecular Weight: $167,21 \mathrm{~g} / \mathrm{mol}$

Following the general procedure above, a mixture of butylamine (S1c) (366 mg, $5.00 \mathrm{mmol}$ ) and CDI $(973 \mathrm{mg}, 6.00 \mathrm{mmol})$ afforded the title compound (2c) as a colorless oil $(690.3 \mathrm{mg}, 82 \%) . R_{f}=0.34$ (dichlormethane/ethyl acetate 1:1); HRMS (ESI) m/z calcd for $\mathrm{C}_{8} \mathrm{H}_{14} \mathrm{~N}_{3} \mathrm{O}[\mathrm{M}+\mathrm{H}]^{+} 168.1131$, found 168.1124; ${ }^{1} \mathrm{H}$ NMR $\left(\left(\mathrm{CD}_{3}\right)_{2} \mathrm{SO}, 400 \mathrm{MHz}\right): \delta 8.46$ (br s, 1H), $8.22(\mathrm{~s}, 1 \mathrm{H}), 7.66(\mathrm{~s}, 1 \mathrm{H}), 7.02(\mathrm{~s}, 1 \mathrm{H})$, $3.28-3.19(\mathrm{~m}, 2 \mathrm{H}), 1.58-1.45(\mathrm{~m}, 2 \mathrm{H}), 1.39-1.25(\mathrm{~m}, 2 \mathrm{H}), 0.90(\mathrm{t}, 3 \mathrm{H}, J=7.3 \mathrm{~Hz}) .{ }^{13} \mathrm{C}$ NMR $\left(\left(\mathrm{CD}_{3}\right)_{2} \mathrm{SO}, 101 \mathrm{MHz}\right): \delta 148.7,135.9,129.5,116.6,31.0,31.0,19.5,13.7$.

d. 1-octyl-carbamoylimidazole (2d)

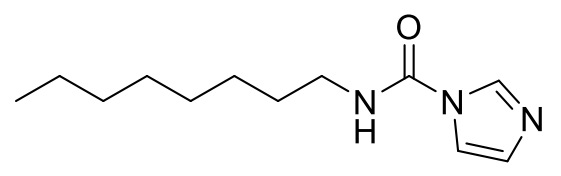

Chemical Formula: $\mathrm{C}_{12} \mathrm{H}_{21} \mathrm{~N}_{3} \mathrm{O}$

Molecular Weight: 223,32 $\mathrm{g} / \mathrm{mol}$

Following the general procedure above, a mixture of octylamine (S1d) $(646 \mathrm{mg}, 5.00 \mathrm{mmol})$ and CDI $(973 \mathrm{mg}, 6.00 \mathrm{mmol})$ afforded the title compound (2d) as a colorless oil $(290.6 \mathrm{mg}, 26 \%) . R_{f}=0.31$ (dichlormethane/ethyl acetate 1:1); HRMS (ESI) m/z calcd for $\mathrm{C}_{12} \mathrm{H}_{22} \mathrm{~N}_{3} \mathrm{O}[\mathrm{M}+\mathrm{H}]^{+} 224.1757$, found 224.1758; ${ }^{1} \mathrm{H}$ NMR $\left(\left(\mathrm{CD}_{3}\right)_{2} \mathrm{SO}, 400 \mathrm{MHz}\right): \delta 8.54-8.40(\mathrm{~m}, 1 \mathrm{H}), 8.22(\mathrm{~s}, 1 \mathrm{H}), 7.66(\mathrm{~s}, 1 \mathrm{H}), 7.01(\mathrm{~s}$, 1H), $3.28-3.17(\mathrm{~m}, 2 \mathrm{H}), 1.59-1.46(\mathrm{~m}, 2 \mathrm{H}), 1.38-1.15(\mathrm{~m}, 10 \mathrm{H}), 0.91-0.79(\mathrm{~m}, 3 \mathrm{H}) .{ }^{13} \mathrm{C}$ NMR $\left(\left(\mathrm{CD}_{3}\right)_{2} \mathrm{SO}, 101 \mathrm{MHz}\right): \delta$ 148.7, 135.9, 129.5, 116.5, 40.1, 31.3, 28.8, 28.6, 26.3, 22.1, 14.0.

e. 1-cyclohexyl-carbamoylimidazole (2e)

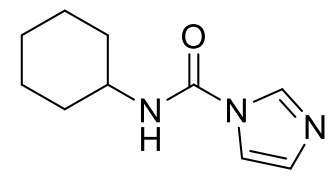

Chemical Formula: $\mathrm{C}_{10} \mathrm{H}_{15} \mathrm{~N}_{3} \mathrm{O}$

Molecular Weight: $193,25 \mathrm{~g} / \mathrm{mol}$

Following the general procedure above, a mixture of cyclohexylamine (S1e) (496 mg, $5.00 \mathrm{mmol})$ and CDI (973 mg, $6.00 \mathrm{mmol}$ ) afforded the title compound (2e) as an off-white powder $(672.2 \mathrm{mg}, 70 \%) . R_{f}$ $=0.27$ (dichlormethane/ethyl acetate 1:1); HRMS (ESI) m/z calcd for $\mathrm{C}_{10} \mathrm{H}_{16} \mathrm{~N}_{3} \mathrm{O}[\mathrm{M}+\mathrm{H}]^{+}$194.1288, found 194.1287; ${ }^{1} \mathrm{H} \mathrm{NMR}\left(\mathrm{CDCl}_{3}, 400 \mathrm{MHz}\right): \delta 8.19(\mathrm{~s}, 1 \mathrm{H}), 7.47(\mathrm{~s}, 1 \mathrm{H}), 7.35(\mathrm{~d}, 1 \mathrm{H}, J=7.5 \mathrm{~Hz}), 6.97$ (s, $1 \mathrm{H}), 3.85-3.69(\mathrm{~m}, 1 \mathrm{H}), 2.10-1.91(\mathrm{~m}, 2 \mathrm{H}), 1.83-1.68(\mathrm{~m}, 2 \mathrm{H}), 1.68-1.55(\mathrm{~m}, 1 \mathrm{H}), 1.42-1.17$ 
$(\mathrm{m}, 4 \mathrm{H}), 1.17-1.02(\mathrm{~m}, 1 \mathrm{H}) .{ }^{13} \mathrm{C} \mathrm{NMR}\left(\mathrm{CDCl}_{3}, 101 \mathrm{MHz}\right): \delta 148.4,136.0,129.6,116.6,50.7,33.0$, $25.4,25.1$.

f. (1H-imidazol-1-yl)(piperidin-1-yl)methanone (2f)

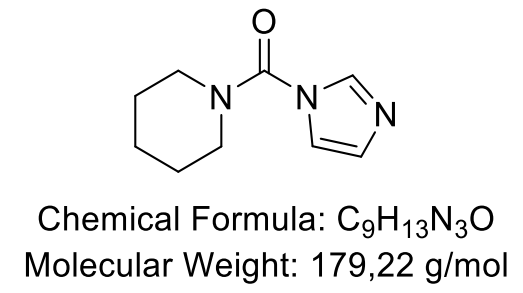

Following the general procedure above, a mixture of piperidine (S1f) (426 mg, $5.00 \mathrm{mmol}$ ) and CDI (973 $\mathrm{mg}, 6.00 \mathrm{mmol})$ afforded the title compound (2f) as an off-white powder $(413.5 \mathrm{mg}, 46 \%) . R_{f}=0.23$ (dichlormethane/ethyl acetate 1:1); ${ }^{1} \mathrm{H} \mathrm{NMR}\left(\mathrm{CDCl}_{3}, 400 \mathrm{MHz}\right): \delta 7.77(\mathrm{~s}, 1 \mathrm{H}), 7.11(\mathrm{~s}, 1 \mathrm{H}), 6.99(\mathrm{~s}$, $1 \mathrm{H}), 3.55-3.34(\mathrm{~m}, 4 \mathrm{H}), 1.76-1.43(\mathrm{~m}, 6 \mathrm{H}) .{ }^{13} \mathrm{C} \mathrm{NMR}\left(\mathrm{CDCl}_{3}, 101 \mathrm{MHz}\right): \delta 150.7,136.8,129.3$, 117.9, 47.4, 25.7, 24.0. NMR data were consistent with literature description. ${ }^{5}$

\section{Synthesis of carbamate-bearing pyranosides 3a-e, 7a-b, and 9a-e}

a. General procedure for the site-selective carbamoylation of pyranosides

A mixture of glycopyranoside $(0.50 \mathrm{mmol}), 1$-carbamoylimidazole $(0.70 \mathrm{mmol})$ and borinic acid $4 \mathbf{b b}^{6}$ $(0.075 \mathrm{mmol})$ in acetonitrile $(5.0 \mathrm{~mL})$ was stirred at reflux for the time indicated in Table 2 . The solvent was then rotary evaporated, and the crude residue was chromatographed over silica gel using a gradient of ethyl acetate (from $10 \%$ to $60 \%$ ) in hexanes as the mobile phase to afford the expected carbamoylation product.

b. Methyl 3-O-benzylcarbamoyl-6-O-(tert-butyldimethyl)silyl- $\alpha$-D-mannopyranoside (3a)

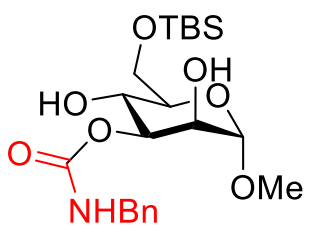

Chemical Formula: $\mathrm{C}_{21} \mathrm{H}_{35} \mathrm{NO}_{7} \mathrm{Si}$

Molecular Weight: $441,60 \mathrm{~g} / \mathrm{mol}$

Following the general procedure above, a mixture of methyl 6-O-(tert-butyldimethyl)silyl- $\alpha$-Dmannopyranoside (1) (154 mg, $0.500 \mathrm{mmol}$ ), 1-benzyl-carbamoylimidazole (2a) (141 mg, $0.700 \mathrm{mmol}$ ) and borinic acid (4b) $)^{6}(15 \mathrm{mg}, 0.075 \mathrm{mmol})$ afforded, after $20 \mathrm{~h}$ of agitation, the title compound (3a) as an off-white powder $(195.8 \mathrm{mg}, 89 \%) . R_{f}=0.39$ (hexanes/ethyl acetate 1:1); $[\alpha]^{20}{ }_{\mathrm{D}}+61.0\left(c 0.1, \mathrm{CH}_{2} \mathrm{Cl}_{2}\right)$; HRMS (ESI) m/z calcd for $\mathrm{C}_{21} \mathrm{H}_{36} \mathrm{NO}_{7} \mathrm{Si}[\mathrm{M}+\mathrm{H}]^{+} 442.2256$, found 442.2257; ${ }^{1} \mathrm{H} \mathrm{NMR}\left(\mathrm{CDCl}_{3}, 400\right.$ MHz): $\delta 7.34-7.20(\mathrm{~m}, 5 \mathrm{H}, \mathrm{ArH}), 5.79(\mathrm{t}, J=5.9 \mathrm{~Hz}, 1 \mathrm{H}, \mathrm{NH}), 4.94(\mathrm{dd}, J=9.7 \mathrm{~Hz}, J=3.1 \mathrm{~Hz}, 1 \mathrm{H}$, $H-3), 4.68$ (br s, $1 \mathrm{H}, H-1), 4.37-4.23$ (m, 2H, $\left.\mathrm{ArCH}_{2}\right), 4.03-3.95$ (m, 1H, H-2), $3.92-3.80$ (m, 3H, 
$H$-4, $H$-6a and $H$-6b), $3.61(\mathrm{dt}, J=9.5 \mathrm{~Hz}, J=4.7 \mathrm{~Hz}, 1 \mathrm{H}, H-5), 3.34$ (s, 3H, OCH3), 2.92 (br s, $1 \mathrm{H}, \mathrm{OH}$ ), 0.89 (s, 9H, $\left.\mathrm{SiC}\left(\mathrm{CH}_{3}\right)_{3}\right), 0.08\left(\mathrm{~s}, 6 \mathrm{H}, \mathrm{Si}\left(\mathrm{CH}_{3}\right)_{2}\right) .{ }^{13} \mathrm{C} \mathrm{NMR}\left(\mathrm{CDCl}_{3}, 101 \mathrm{MHz}\right): \delta 157.1,138.2,128.7$, $127.7,127.6,100.7,72.3,69.5,67.7,64.0,54.9,45.2,26.0,18.4,-5.3$.

c. Synthesis of compound $\mathbf{3 a}$ on a $2 \mathrm{mmol}$ scale

A mixture of methyl 6-O-(tert-butyldimethyl)silyl- $\alpha$-D-mannopyranoside (1) $(617 \mathrm{mg}, 2.0 \mathrm{mmol}), 1$ benzyl-carbamoylimidazole (2a) $(604 \mathrm{mg}, 3.0 \mathrm{mmol})$ and 2-aminoethyl diphenylborinate (4a) (90.0 $\mathrm{mg}$, $0.40 \mathrm{mmol})$ in acetonitrile $(20 \mathrm{~mL})$ was stirred at reflux for $20 \mathrm{~h}$. The solvent was then rotary evaporated, and the crude residue was chromatographed over silica gel using a gradient of ethyl acetate (from 10\% to $60 \%$ ) in hexanes as the mobile phase to afford the title compound (3a) as an off-white powder (655 $\mathrm{mg}, 74 \%)$.

d. Methyl 3-O-methylcarbamoyl-6-O-(tert-butyldimethyl)silyl- $\alpha$-D-mannopyranoside (3b)

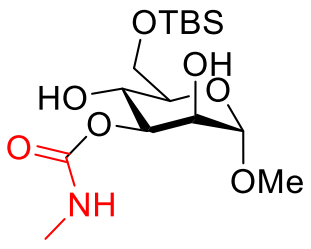

Chemical Formula: $\mathrm{C}_{15} \mathrm{H}_{31} \mathrm{NO}_{7} \mathrm{Si}$

Molecular Weight: $365,50 \mathrm{~g} / \mathrm{mol}$

Following the general procedure above, a mixture of methyl 6-O-(tert-butyldimethyl)silyl- $\alpha$-Dmannopyranoside (1) (154 mg, $0.500 \mathrm{mmol})$, 1-methyl-carbamoylimidazole (2b) ${ }^{7}$ (88 $\mathrm{mg}, 0.70 \mathrm{mmol}$ ) and borinic acid (4b) $(15 \mathrm{mg}, 0.075 \mathrm{mmol})$ afforded, after $20 \mathrm{~h}$ of agitation, the title compound (3b) as an off-white powder $(119.4 \mathrm{mg}, 65 \%) . R_{f}=0.18$ (chloroform/methanol 9:1); $[\alpha]^{20} \mathrm{D}-120.0(c 0.1$, $\mathrm{CH}_{2} \mathrm{Cl}_{2}$ ); HRMS (ESI) $\mathrm{m} / \mathrm{z}$ calcd for $\mathrm{C}_{15} \mathrm{H}_{32} \mathrm{NO}_{7} \mathrm{Si}[\mathrm{M}+\mathrm{H}]^{+} 366.1943$, found 366.1934; ${ }^{1} \mathrm{H} \mathrm{NMR}$ $\left(\mathrm{CDCl}_{3}, 400 \mathrm{MHz}\right): \delta 5.86(\mathrm{br} \mathrm{s}, 1 \mathrm{H}, \mathrm{NH}), 4.91-4.79(\mathrm{~m}, 1 \mathrm{H}, H-3), 4.72-4.60$ (m, 1H, H-1), $4.04-$ $3.70(\mathrm{~m}, 6 \mathrm{H}, H-2, H-4, H-6 \mathrm{a}$ and $H-6 \mathrm{~b}), 3.62-3.53(\mathrm{~m}, 1 \mathrm{H}, H-5), 3.33\left(\mathrm{~s}, 3 \mathrm{H}, \mathrm{OCH}_{3}\right), 2.72(\mathrm{~s}, 3 \mathrm{H}$, $\left.\mathrm{NHCH}_{3}\right), 0.86\left(\mathrm{~s}, 9 \mathrm{H}, \mathrm{SiC}\left(\mathrm{CH}_{3}\right)_{3}\right), 0.05\left(\mathrm{~s}, 6 \mathrm{H}, \mathrm{Si}\left(\mathrm{CH}_{3}\right)_{2}\right) .{ }^{13} \mathrm{C} \mathrm{NMR}\left(\mathrm{CDCl}_{3}, 101 \mathrm{MHz}\right): \delta 157.7,100.7$, $75.1,72.5,69.4,67.2,63.9,54.8,27.6,25.9,18.3,-5.4,-5.4$.

e. Methyl 3-O-butylcarbamoyl-6-O-(tert-butyldimethyl)silyl- $\alpha$-D-mannopyranoside (3c)

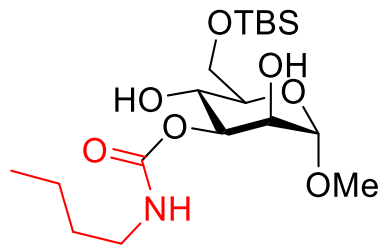

Chemical Formula: $\mathrm{C}_{18} \mathrm{H}_{37} \mathrm{NO}_{7} \mathrm{Si}$

Molecular Weight: $407,58 \mathrm{~g} / \mathrm{mol}$

Following the general procedure above, a mixture of methyl 6-O-(tert-butyldimethyl)silyl- $\alpha$-Dmannopyranoside (1) (154 mg, $0.500 \mathrm{mmol}$ ), 1-butyl-carbamoylimidazole (2c) (117 mg, $0.700 \mathrm{mmol})$ and borinic acid (4b) (15 mg, $0.075 \mathrm{mmol})$ afforded, after $48 \mathrm{~h}$ of agitation, the title compound (3c) as 
an colorless oil $(140.5 \mathrm{mg}, 69 \%) . R_{f}=0.32$ (dichloromethane/ethyl acetate $\left.1: 1\right) ;[\alpha]^{20} \mathrm{D}+70.0(c 0.1$, $\mathrm{CH}_{2} \mathrm{Cl}_{2}$ ); HRMS (ESI) $\mathrm{m} / \mathrm{z}$ calcd for $\mathrm{C}_{18} \mathrm{H}_{38} \mathrm{NO}_{7} \mathrm{Si}[\mathrm{M}+\mathrm{H}]^{+}$408.2412, found 408.2409; ${ }^{1} \mathrm{H}$ NMR $\left(\mathrm{CDCl}_{3}, 400 \mathrm{MHz}\right): \delta 5.11$ (br s, $\left.1 \mathrm{H}, \mathrm{NH}\right), 4.92(\mathrm{dd}, 1 \mathrm{H}, J=9.5 \mathrm{~Hz}, J=2.2 \mathrm{~Hz}, H-3), 4.70($ br s, $1 \mathrm{H}, H-$ 1), 4.00 (br s, $1 \mathrm{H}, H-2), 3.95-3.85$ (m, H, $H-4, H-6 \mathrm{a}$ and $H-6 \mathrm{~b}), 3.69-3.57$ (m, $1 \mathrm{H}, H-5), 3.38$ (s, $3 \mathrm{H}$, $\left.\mathrm{OCH}_{3}\right), 3.18\left(\mathrm{t}, 2 \mathrm{H}, J=6.9 \mathrm{~Hz}, \mathrm{NHCH}_{2}\right), 1.57-1.43\left(\mathrm{~m}, 2 \mathrm{H}, \mathrm{NHCH}_{2} \mathrm{CH}_{2} \mathrm{CH}_{2} \mathrm{CH}_{3}\right), 1.43-1.26(\mathrm{~m}, 2 \mathrm{H}$, $\left.\mathrm{NHCH}_{2} \mathrm{CH}_{2} \mathrm{CH}_{2} \mathrm{CH}_{3}\right), 0.99-0.83$ (m, 3H, $\left.\mathrm{NHCH}_{2} \mathrm{CH}_{2} \mathrm{CH}_{2} \mathrm{CH}_{3}\right), 0.90$ (s, 9H, SiC(CH3) $), 0.09$ (s, 6H, $\left.\mathrm{Si}\left(\mathrm{CH}_{3}\right)_{2}\right) .{ }^{13} \mathrm{C} \mathrm{NMR}\left(\mathrm{CDCl}_{3}, 101 \mathrm{MHz}\right): \delta$ 157.0, 100.7, 75.4, 72.2, 69.8, 67.8, 63.9, 55.1, 41.1, 31.9, 26.0, 20.0, 18.4, 13.8, -5.3.

f. Methyl 3-O-octylcarbamoyl-6-O-(tert-butyldimethyl)silyl- $\alpha$-D-mannopyranoside (3d)

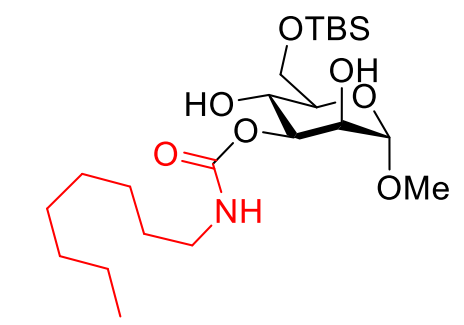

Chemical Formula: $\mathrm{C}_{22} \mathrm{H}_{45} \mathrm{NO}_{7} \mathrm{Si}$

Molecular Weight: 463,69 g/mol

Following the general procedure above, a mixture of methyl 6-O-(tert-butyldimethyl)silyl- $\alpha$-Dmannopyranoside (1) (154 mg, $0.500 \mathrm{mmol})$, 1-octyl-carbamoylimidazole (2d) (156 mg, $0.700 \mathrm{mmol})$ and borinic acid (4b) $(15 \mathrm{mg}, 0.075 \mathrm{mmol})$ afforded, after $48 \mathrm{~h}$ of agitation, the title compound (3d) as an colorless oil $(170.2 \mathrm{mg}, 73 \%) . R_{f}=0.84$ (dichloromethane/ethyl acetate $\left.1: 1\right) ;[\alpha]^{20} \mathrm{D}+67.0(c 0.1$, $\mathrm{CH}_{2} \mathrm{Cl}_{2}$ ); HRMS (ESI) $\mathrm{m} / \mathrm{z}$ calcd for $\mathrm{C}_{22} \mathrm{H}_{46} \mathrm{NO}_{7} \mathrm{Si}[\mathrm{M}+\mathrm{H}]^{+} 464.3038$, found 464.3030; ${ }^{1} \mathrm{H} \mathrm{NMR}$ $\left(\mathrm{CDCl}_{3}, 400 \mathrm{MHz}\right): \delta 5.08$ (br s, $\left.1 \mathrm{H}, \mathrm{NH}\right), 4.91(\mathrm{dd}, 1 \mathrm{H}, J=9.6 \mathrm{~Hz}, J=3.1 \mathrm{~Hz}, H-3), 4.70$ (br s, $1 \mathrm{H}, H$ 1), $4.06-3.97$ (m, $1 \mathrm{H}, H-2), 3.97-3.84$ (m, 3H, $H-4, H-6 \mathrm{a}$ and $H-6 \mathrm{~b}), 3.67-3.58$ (m, $1 \mathrm{H}, H-5), 3.38$ $\left(\mathrm{s}, 3 \mathrm{H}, \mathrm{OCH}_{3}\right), 3.23-3.13\left(\mathrm{~m}, 2 \mathrm{H}, \mathrm{NHCH}_{2}\right), 1.57-1.43\left(\mathrm{~m}, 2 \mathrm{H}, \mathrm{NHCH}_{2} \mathrm{CH}_{2}\left(\mathrm{CH}_{2}\right)_{5} \mathrm{CH}_{3}\right), 1.38-1.19$ $\left(\mathrm{m}, 10 \mathrm{H}, \mathrm{NH}\left(\mathrm{CH}_{2}\right)_{2}\left(\mathrm{CH}_{2}\right)_{5} \mathrm{CH}_{3}\right), 1.02-0.79\left(\mathrm{~m}, 3 \mathrm{H}, \mathrm{NH}\left(\mathrm{CH}_{2}\right)_{7} \mathrm{CH}_{3}\right), 0.90\left(\mathrm{~s}, 9 \mathrm{H}, \mathrm{SiC}\left(\mathrm{CH}_{3}\right)_{3}\right), 0.09$ (s, $\left.6 \mathrm{H}, \mathrm{Si}\left(\mathrm{CH}_{3}\right)_{2}\right) .{ }^{13} \mathrm{C} \mathrm{NMR}\left(\mathrm{CDCl}_{3}, 101 \mathrm{MHz}\right): \delta 157.0,100.7,75.4,72.2,69.8,67.8,64.0,55.0,41.4$, $31.9,29.9,29.3,29.3,26.9,26.0,22.8,18.4,14.2,-5.3$.

g. Methyl 3-O-cyclohexylcarbamoyl-6-O-(tert-butyldimethyl)silyl- $\alpha$-D-mannopyranoside (3e)

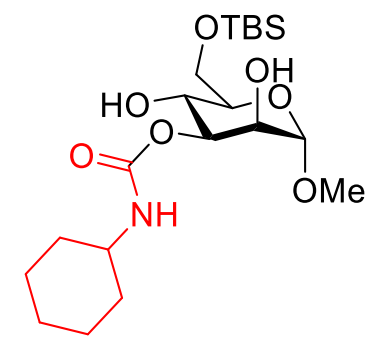

Chemical Formula: $\mathrm{C}_{20} \mathrm{H}_{39} \mathrm{NO}_{7} \mathrm{Si}$

Molecular Weight: $433,62 \mathrm{~g} / \mathrm{mol}$ 
Following the general procedure above, a mixture of methyl 6-O-(tert-butyldimethyl)silyl- $\alpha$-Dmannopyranoside (1) (154 mg, $0.500 \mathrm{mmol}$ ), 1-cyclohexyl-carbamoylimidazole (2e) (135 mg, 0.700 $\mathrm{mmol}$ ) and borinic acid (4b) $(15 \mathrm{mg}, 0.075 \mathrm{mmol})$ afforded, after $20 \mathrm{~h}$ of agitation, the title compound (3e) as an off-white powder $(167.0 \mathrm{mg}, 77 \%) . R_{f}=0.44$ (dichloromethane/ethyl acetate $7: 3$ ); $[\alpha]^{20} \mathrm{D}-2.8$ (c $0.1, \mathrm{CH}_{2} \mathrm{Cl}_{2}$ ); HRMS (ESI) $\mathrm{m} / \mathrm{z}$ calcd for $\mathrm{C}_{20} \mathrm{H}_{40} \mathrm{NO}_{7} \mathrm{Si}[\mathrm{M}+\mathrm{H}]^{+} 434.2569$, found 434.2562; ${ }^{1} \mathrm{H} \mathrm{NMR}$ $\left(\mathrm{CDCl}_{3}, 400 \mathrm{MHz}\right): \delta 5.02(\mathrm{~d}, 1 \mathrm{H}, J=7.3 \mathrm{~Hz}, \mathrm{NH}), 4.89(\mathrm{dd}, 1 \mathrm{H}, J=9.7 \mathrm{~Hz}, J=2.3 \mathrm{~Hz}, H-3), 4.70$ (s, $1 \mathrm{H}, H-1$ ), 3.99 (br s, $1 \mathrm{H}, H-2), 3.94-3.84$ (m, 3H, $H-4, H-6 \mathrm{a}$ and $H-6 \mathrm{~b}$ ), $3.66-3.57$ (m, $1 \mathrm{H}, H-5), 3.55$ - $3.42(\mathrm{~m}, 1 \mathrm{H}, \mathrm{NHCH}), 3.38\left(\mathrm{~s}, 3 \mathrm{H}, \mathrm{OCH}_{3}\right), 1.99-1.87(\mathrm{~m}, 2 \mathrm{H}, \mathrm{Cyclohexyl} H), 1.76-1.64(\mathrm{~m}, 2 \mathrm{H}$, Cyclohexyl $H$ ), $1.64-1.53(\mathrm{~m}, 1 \mathrm{H}$, Cyclohexyl $H), 1.41-1.26(\mathrm{~m}, 2 \mathrm{H}$, Cyclohexyl $H), 1.22-1.06(\mathrm{~m}$, $3 \mathrm{H}), 0.90$ (s, 9H, $\left.\mathrm{SiC}\left(\mathrm{CH}_{3}\right)_{3}\right), 0.09$ (s, 6H, Si $\left.\left(\mathrm{CH}_{3}\right)_{2}\right) .{ }^{13} \mathrm{C} \mathrm{NMR}\left(\mathrm{CDCl}_{3}, 101 \mathrm{MHz}\right): \delta 156.2,100.7,75.3$, $72.4,69.8,67.7,63.9,55.0,50.3,33.3,33.2,26.0,25.5,24.8,18.4,-5.3$.

h. Methyl 3-O-benzylcarbamoyl-6-O-(tert-butyldimethyl)silyl- $\beta$-D-galactopyranoside (7a)

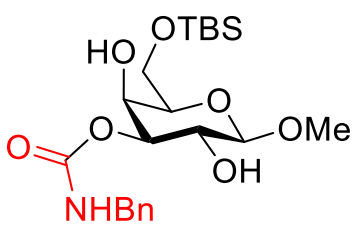

Chemical Formula: $\mathrm{C}_{21} \mathrm{H}_{35} \mathrm{NO}_{7} \mathrm{Si}$

Molecular Weight: $441,60 \mathrm{~g} / \mathrm{mol}$

Following the general procedure above, a mixture of methyl 6-O-(tert-butyldimethyl)silyl- $\beta$-Dgalactopyranoside (6a) (154 mg, $0.500 \mathrm{mmol}$ ), 1-benzyl-carbamoylimidazole (2a) (141 mg, $0.700 \mathrm{mmol}$ ) and borinic acid (4b) $(15 \mathrm{mg}, 0.075 \mathrm{mmol})$ afforded, after $20 \mathrm{~h}$ of agitation, the title compound (7a) as an colorless oil (171.9 mg, 78\%). $R_{f}=0.40$ (hexanes/ethyl acetate 1:1); $[\alpha]^{20}{ }_{\mathrm{D}}-18.7\left(c 0.1, \mathrm{CH}_{2} \mathrm{Cl}_{2}\right)$; HRMS (ESI) $\mathrm{m} / \mathrm{z}$ calcd for $\mathrm{C}_{21} \mathrm{H}_{36} \mathrm{NO}_{7} \mathrm{Si}[\mathrm{M}+\mathrm{H}]^{+}$442.2256, found 442.2254; ${ }^{1} \mathrm{H} \mathrm{NMR}\left(\mathrm{CDCl}_{3}, 400\right.$ $\mathrm{MHz}): \delta 7.34-7.16(\mathrm{~m}, 5 \mathrm{H}, \mathrm{ArH}), 6.03(\mathrm{t}, 1 \mathrm{H}, J=5.9 \mathrm{~Hz}, \mathrm{NH}), 4.69(\mathrm{dd}, 1 \mathrm{H}, J=10.0 \mathrm{~Hz}, J=2.9 \mathrm{~Hz}$, $H-3), 4.36-4.23$ (m, 2H, $\left.\mathrm{ArCH}_{2}\right), 4.23-4.12$ (m, 2H, H-1, H-4), $3.94-3.76$ (m, 3H, H-2, H-6a and $H-$ 6b), $3.50-3.40(\mathrm{~m}, 1 \mathrm{H}, \mathrm{H}-5), 3.44\left(\mathrm{~s}, 3 \mathrm{H}, \mathrm{OCH}_{3}\right), 0.87\left(\mathrm{~s}, 9 \mathrm{H}, \mathrm{SiC}\left(\mathrm{CH}_{3}\right)_{3}\right), 0.05\left(\mathrm{~s}, 6 \mathrm{H}, \mathrm{Si}\left(\mathrm{CH}_{3}\right)_{2}\right) .{ }^{13} \mathrm{C}$ NMR $\left(\mathrm{CDCl}_{3}, 101 \mathrm{MHz}\right): \delta 156.4,138.4,128.6,127.6,127.4,104.3,76.3,74.0,69.4,68.2,62.9,57.0$, $45.1,25.9,18.3,-5.4$.

i. Methyl 3-O-benzylcarbamoyl-6-O-(tert-butyldimethyl)silyl- $\alpha$-D-galactopyranoside (7b)

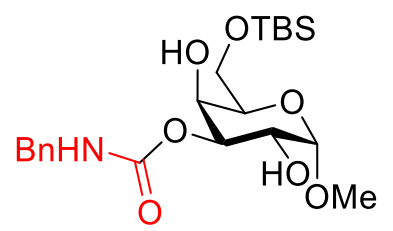

Chemical Formula: $\mathrm{C}_{21} \mathrm{H}_{35} \mathrm{NO}_{7} \mathrm{Si}$

Molecular Weight: $441,60 \mathrm{~g} / \mathrm{mol}$

Following the general procedure above, a mixture of methyl 6-O-(tert-butyldimethyl)silyl- $\alpha$-Dgalactopyranoside (6b) (154 mg, $0.500 \mathrm{mmol}$ ), 1-benzyl-carbamoylimidazole (2a) (141 mg, $0.700 \mathrm{mmol}$ ) and borinic acid (4b) (15 mg, $0.075 \mathrm{mmol})$ afforded, after $20 \mathrm{~h}$ of agitation, the title compound (7b) as 
an off-white powder $(153.3 \mathrm{mg}, 69 \%) . R_{f}=0.40$ (hexanes/ethyl acetate 1:1); $[\alpha]^{20} \mathrm{D}+103.3(c 0.1$, $\mathrm{CH}_{2} \mathrm{Cl}_{2}$ ); HRMS (ESI) $\mathrm{m} / \mathrm{z}$ calcd for $\mathrm{C}_{21} \mathrm{H}_{36} \mathrm{NO}_{7} \mathrm{Si}[\mathrm{M}+\mathrm{H}]^{+} 442.2256$, found 442.2255; ${ }^{1} \mathrm{H} \mathrm{NMR}$ $\left(\mathrm{CDCl}_{3}, 400 \mathrm{MHz}\right): \delta 7.40-7.12(\mathrm{~m}, 5 \mathrm{H}, \mathrm{ArH}), 5.85(\mathrm{t}, 1 \mathrm{H}, J=5.8 \mathrm{~Hz}, \mathrm{NH}), 4.93(\mathrm{dd}, 1 \mathrm{H}, J=10.3 \mathrm{~Hz}$, $J=2.7 \mathrm{~Hz}, H-3), 4.77$ (d, $1 \mathrm{H}, J=3.8 \mathrm{~Hz}, H-1), 4.38-4.25$ (m, 2H, ArCH $_{2}, 4.19$ (br s, $\left.1 \mathrm{H}, H-4\right), 4.14-$ $4.03(\mathrm{~m}, 1 \mathrm{H}, H-2), 3.90-3.79$ (m, 2H, $H$-6a and $H$-6b), $3.78-3.71$ (m, $1 \mathrm{H}, H-5), 3.60$ (br s, $1 \mathrm{H}, \mathrm{OH})$, $3.38\left(\mathrm{~s}, 3 \mathrm{H}, \mathrm{OCH}_{3}\right), 2.61$ (br s, $\left.1 \mathrm{H}, \mathrm{OH}\right), 0.88$ (s, 9H, $\left.\mathrm{SiC}\left(\mathrm{CH}_{3}\right)_{3}\right), 0.07$ (s, 6H, $\left.\mathrm{Si}\left(\mathrm{CH}_{3}\right)_{2}\right) .{ }^{13} \mathrm{C} \mathrm{NMR}$ $\left(\mathrm{CDCl}_{3}, 101 \mathrm{MHz}\right): \delta 156.6,138.4,128.6,127.6,127.4,99.9,74.1,69.5,69.5,67.4,63.8,55.4,45.1$, $25.9,18.3,-5.4$.

j. 4-Methoxyphenyl 3-O-benzylcarbamoyl-6-O-(tert-butyldimethyl)silyl- $\alpha$-Dmannopyranoside (9a)

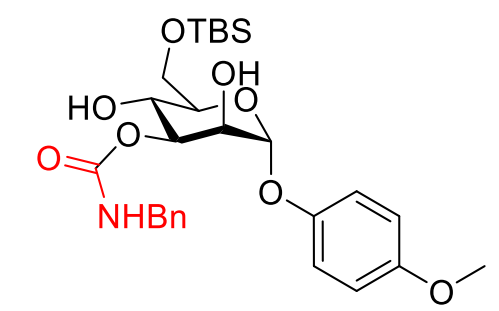

Chemical Formula: $\mathrm{C}_{27} \mathrm{H}_{39} \mathrm{NO}_{8} \mathrm{Si}$

Molecular Weight: $533,69 \mathrm{~g} / \mathrm{mol}$

Following the general procedure above, a mixture of 4-methoxyphenyl 6-O-(tert-butyldimethyl)silyl- $\alpha$ D-mannopyranoside (8a) (201 mg, $0.500 \mathrm{mmol}$ ), 1-benzyl-carbamoylimidazole (2a) (141 mg, 0.700 $\mathrm{mmol}$ ) and borinic acid (4b) $(15 \mathrm{mg}, 0.075 \mathrm{mmol})$ afforded, after $20 \mathrm{~h}$ of agitation, the title compound (9a) as an off-white powder $(186.5 \mathrm{mg}, 70 \%) . R_{f}=0.52$ (dichloromethane/ethyl acetate 7:3); $[\alpha]^{20} \mathrm{D}^{+}$ 18.0 ( c 0.1, $\mathrm{CH}_{2} \mathrm{Cl}_{2}$ ); HRMS (ESI) m/z calcd for $\mathrm{C}_{27} \mathrm{H}_{40} \mathrm{NO}_{8} \mathrm{Si}[\mathrm{M}+\mathrm{H}]^{+} 534.2518$, found 534.2514; ${ }^{1} \mathrm{H}$ NMR $\left(\mathrm{CDCl}_{3}, 400 \mathrm{MHz}\right): \delta 7.40-7.24(\mathrm{~m}, 5 \mathrm{H}, \mathrm{ArH}), 7.01(\mathrm{~d}, 2 \mathrm{H}, J=8.9 \mathrm{~Hz}, \mathrm{ArH}), 6.81(\mathrm{~d}, 2 \mathrm{H}, J=$ $8.9 \mathrm{~Hz}, \mathrm{ArH}), 5.55-5.45$ (m, 1H, NH), 5.40 (br s, $1 \mathrm{H}, H-1), 5.21$ (dd, $1 \mathrm{H}, J=9.7 \mathrm{~Hz}, J=2.8 \mathrm{~Hz}, H-3$ ), $4.46-4.32$ (m, 2H, $\left.\mathrm{ArCH}_{2}\right), 4.23$ (br s, $\left.1 \mathrm{H}, H-2\right), 4.13-3.96(\mathrm{~m}, 1 \mathrm{H}, H-4), 3.90-3.79$ (m, 3H, $H-5, H-$ 6a and $\mathrm{H}-6 \mathrm{~b}), 3.77$ (s, 3H, $\left.\mathrm{OCH}_{3}\right), 0.87$ (s, 9H, $\left.\mathrm{SiC}\left(\mathrm{CH}_{3}\right)_{3}\right), 0.05\left(\mathrm{~s}, 6 \mathrm{H}, \mathrm{Si}\left(\mathrm{CH}_{3}\right)_{2}\right)$. ${ }^{13} \mathrm{C} \mathrm{NMR}\left(\mathrm{CDCl}_{3}\right.$, $101 \mathrm{MHz}): \delta 156.9,155.2,150.3,138.0,128.9,127.8,117.9,114.7,98.6,75.3,72.5,69.7,67.9,64.1$, $55.8,45.5,26.0,18.4,-5.3,-5.3$.

k. 4-Methoxyphenyl galactopyranoside (9b)

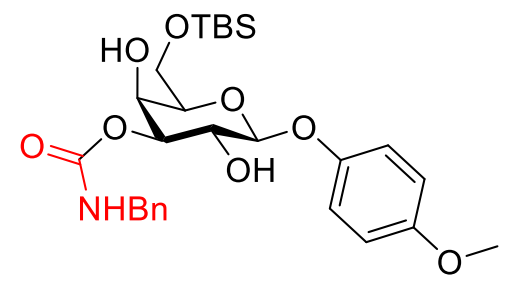

Chemical Formula: $\mathrm{C}_{27} \mathrm{H}_{39} \mathrm{NO}_{8} \mathrm{Si}$

Molecular Weight: $533,69 \mathrm{~g} / \mathrm{mol}$ 
Following the general procedure above, a mixture of 4-methoxyphenyl 6-O-(tert-butyldimethyl)silyl- $\beta$ D-galactopyranoside (8b) (201 mg, $0.500 \mathrm{mmol}$ ), 1-benzyl-carbamoylimidazole (2a) (141 mg, 0.700 $\mathrm{mmol}$ ) and borinic acid (4b) $(15 \mathrm{mg}, 0.075 \mathrm{mmol})$ afforded, after $20 \mathrm{~h}$ of agitation, the title compound (9b) as an off-white powder $(218.7 \mathrm{mg}, 82 \%) . R_{f}=0.53$ (dichloromethane/ethyl acetate 7:3); $[\alpha]^{20}{ }_{\mathrm{D}}-$ 107.6 ( $c$ 0.1, $\mathrm{CH}_{2} \mathrm{Cl}_{2}$ ); HRMS (ESI) m/z calcd for $\mathrm{C}_{27} \mathrm{H}_{40} \mathrm{NO}_{8} \mathrm{Si}[\mathrm{M}+\mathrm{H}]^{+} 534.2518$, found 534.2516; ${ }^{1} \mathrm{H}$ NMR (( $\left.\mathrm{CDCl}_{3}, 400 \mathrm{MHz}\right): \delta 7.36-7.24(\mathrm{~m}, 5 \mathrm{H}, \mathrm{ArH}), 7.03(\mathrm{~d}, 2 \mathrm{H}, J=9.0 \mathrm{~Hz}, \mathrm{ArH}), 6.80(\mathrm{~d}, 2 \mathrm{H}, J=$ $9.0 \mathrm{~Hz}, \mathrm{ArH}), 5.56-5.47$ (m, 1H, NH), $4.88-4.75$ (m, 2H, H-1, H-3), $4.40-4.33$ (m, 2H, ArCH $H_{2}, 4.27$ $-4.21(\mathrm{~m}, 1 \mathrm{H}, H-4), 4.17-4.08(\mathrm{~m}, 1 \mathrm{H}, H-2), 3.93-3.85(\mathrm{~m}, 2 \mathrm{H}, H-6 \mathrm{a}$ and $H-6 \mathrm{~b}), 3.77\left(\mathrm{~s}, 3 \mathrm{H}, \mathrm{OCH}_{3}\right)$, $3.62-3.55(\mathrm{~m}, 1 \mathrm{H}, \mathrm{H}-5), 0.88\left(\mathrm{~s}, 9 \mathrm{H}, \mathrm{SiC}\left(\mathrm{CH}_{3}\right)_{3}\right), 0.14-0.02\left(\mathrm{~m}, 6 \mathrm{H}, \mathrm{Si}\left(\mathrm{CH}_{3}\right)_{2}\right) .{ }^{13} \mathrm{C} \mathrm{NMR}\left(\mathrm{CDCl}_{3}, 101\right.$ $\mathrm{MHz}): \delta 156.3,155.6,151.3,138.1,128.8,127.7,119.0,114.6,103.1,76.2,74.1,69.6,68.7,63.3,55.8$, $45.4,25.9,18.3,-5.4,-5.4$.

1. 4-Methoxyphenyl 3-O-benzylcarbamoyl- $\alpha$-L-arabinopyranoside (9c)

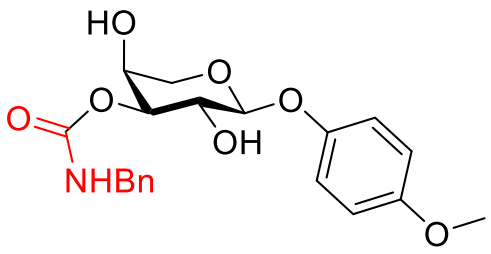

Chemical Formula: $\mathrm{C}_{20} \mathrm{H}_{23} \mathrm{NO}_{7}$

Molecular Weight: $389,40 \mathrm{~g} / \mathrm{mol}$

Following the general procedure above, a mixture of 4-methoxyphenyl $\alpha$ - $L$-arabinopyranoside ${ }^{8}(\mathbf{8 c})(128$ $\mathrm{mg}, 0.500 \mathrm{mmol})$, 1-benzyl-carbamoylimidazole (2a) $(141 \mathrm{mg}, 0.700 \mathrm{mmol})$ and borinic acid (4b) (15 $\mathrm{mg}, 0.075 \mathrm{mmol})$ afforded, after $48 \mathrm{~h}$ of agitation, the title compound (9c) as an off-white powder (141.0 $\mathrm{mg}, 72 \%) . R_{f}=0.43$ (dichloromethane/ethyl acetate $\left.1: 1\right) ;[\alpha]^{20}+113.4\left(c 0.1, \mathrm{CH}_{2} \mathrm{Cl}_{2}\right) ; \mathrm{HRMS}$ (ESI) $\mathrm{m} / \mathrm{z}$ calcd for $\mathrm{C}_{20} \mathrm{H}_{24} \mathrm{NO}_{7}[\mathrm{M}+\mathrm{H}]^{+} 390.1547$, found 390.1548; ${ }^{1} \mathrm{H} \mathrm{NMR}\left(\mathrm{CDCl}_{3}, 400 \mathrm{MHz}\right): \delta 7.42-$ $7.11(\mathrm{~m}, 5 \mathrm{H}, \operatorname{Ar} H), 6.96(\mathrm{~d}, 2 \mathrm{H}, J=9.0 \mathrm{~Hz}, \operatorname{Ar} H), 6.80(\mathrm{~d}, 2 \mathrm{H}, J=9.0 \mathrm{~Hz}, \operatorname{Ar} H), 6.11(\mathrm{t}, J=5.7 \mathrm{~Hz}, 1 \mathrm{H}$, $\mathrm{NH}), 5.39(\mathrm{~d}, 1 \mathrm{H}, J=3.5 \mathrm{~Hz}, H-1), 5.17(\mathrm{dd}, 1 \mathrm{H}, J=10.3 \mathrm{~Hz}, J=2.8 \mathrm{~Hz}, H-3), 4.40-4.23(\mathrm{~m}, 2 \mathrm{H}$, $\left.\mathrm{ArCH}_{2}\right), 4.23-4.14(\mathrm{~m}, 1 \mathrm{H}, \mathrm{H}-2), 4.14-4.04(\mathrm{~m}, 1 \mathrm{H}, H-4), 3.99-3.86(\mathrm{~m}, 1 \mathrm{H}, H-6 \mathrm{a}), 3.75$ (s, $3 \mathrm{H}$, $\left.\mathrm{OCH}_{3}\right), 3.70-3.59(\mathrm{~m}, 1 \mathrm{H}, \mathrm{H}-6 \mathrm{~b}), 3.59-3.41(\mathrm{br}, 1 \mathrm{H}, \mathrm{OH}), 3.21-2.99(\mathrm{br}, 1 \mathrm{H}, \mathrm{OH}) .{ }^{13} \mathrm{C} \mathrm{NMR}\left(\mathrm{CDCl}_{3}\right.$, $101 \mathrm{MHz}): \delta 156.7,155.3,150.5,138.3,128.7,127.6,118.1,114.7,98.9,73.3,68.4,67.5,63.3,55.7$, 45.1.

m. 4-Methoxyphenyl 3-O-benzylcarbamoyl- $\alpha$-L-lyxopyranoside (9d)

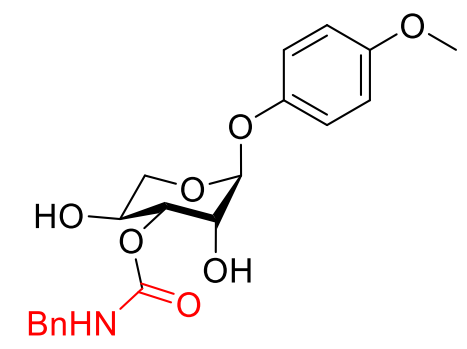

Chemical Formula: $\mathrm{C}_{20} \mathrm{H}_{23} \mathrm{NO}_{7}$

Molecular Weight: $389,40 \mathrm{~g} / \mathrm{mol}$ 
Following the general procedure above, a mixture of 4-methoxyphenyl $\alpha$-L-lyxopyranoside ${ }^{9}$ (8d) (128 $\mathrm{mg}, 0.500 \mathrm{mmol})$, 1-benzyl-carbamoylimidazole (2a) $(141 \mathrm{mg}, 0.700 \mathrm{mmol})$ and borinic acid (4b) (15 $\mathrm{mg}, 0.075 \mathrm{mmol}$ ) afforded, after $48 \mathrm{~h}$ of agitation, the title compound (9d) as an off-white powder (109.5 $\mathrm{mg}, 56 \%$ ). $R_{f}=0.43$ (dichloromethane/ethyl acetate 1:1); $[\alpha]^{20} \mathrm{D}-119.8\left(c 0.1, \mathrm{CH}_{2} \mathrm{Cl}_{2}\right) ; \mathrm{HRMS}$ (ESI) $\mathrm{m} / \mathrm{z}$ calcd for $\mathrm{C}_{20} \mathrm{H}_{24} \mathrm{NO}_{7}[\mathrm{M}+\mathrm{H}]+390.1547$, found 390.1546; ${ }^{1} \mathrm{H} \mathrm{NMR}\left(\mathrm{CD}_{3} \mathrm{OD}, 400 \mathrm{MHz}\right): \delta 7.43-$ $7.18(\mathrm{~m}, 5 \mathrm{H}, \operatorname{Ar} H), 7.01(\mathrm{~d}, 2 \mathrm{H}, J=8.9 \mathrm{~Hz}, \operatorname{ArH}), 6.84(\mathrm{~d}, 2 \mathrm{H}, J=8.9 \mathrm{~Hz}, \operatorname{Ar} H), 5.29(\mathrm{~d}, 1 \mathrm{H}, J=2.4 \mathrm{~Hz}$, $H-1), 5.05(\mathrm{dd}, 1 \mathrm{H}, J=8.8 \mathrm{~Hz}, J=3.0 \mathrm{~Hz}, H-3), 4.42-4.25\left(\mathrm{~m}, 2 \mathrm{H}, \mathrm{ArCH}_{2}\right), 4.23-4.12(\mathrm{~m}, 1 \mathrm{H}, H-2)$, $4.11-3.99$ (m, 1H, H-4), $3.84-3.68$ (m, $1 \mathrm{H}, H-5 \mathrm{a}), 3.73$ (s, $\left.3 \mathrm{H}, \mathrm{OCH}_{3}\right), 3.68-3.59$ (m, $\left.1 \mathrm{H}, H-5 \mathrm{~b}\right) .{ }^{13} \mathrm{C}$ NMR (CD $\left.{ }_{3} \mathrm{OD}, 101 \mathrm{MHz}\right): \delta 158.5,156.6,151.8,140.4,129.5,128.4,128.2,119.0,115.6,101.0,76.0$, $69.8,66.0,64.8,56.0,45.5$.

n. 4-Methoxyphenyl 3-O-benzylcarbamoyl- $\alpha$-L-rhamnopyranoside (9e)

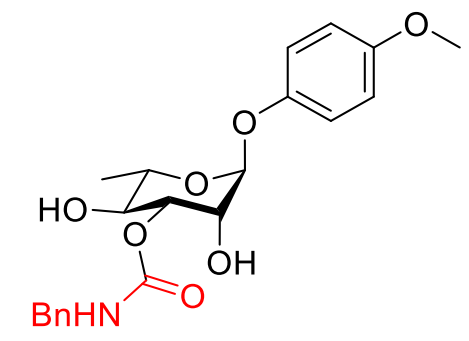

Chemical Formula: $\mathrm{C}_{21} \mathrm{H}_{25} \mathrm{NO}_{7}$

Molecular Weight: 403,43 g/mol

Following the general procedure above, a mixture of 4-methoxyphenyl $\alpha$-L-rhamnopyranoside ${ }^{10}(\mathbf{8 e})$ (135 mg, $0.500 \mathrm{mmol}$ ), 1-benzyl-carbamoylimidazole (2a) (141 mg, $0.700 \mathrm{mmol}$ ) and borinic acid (4b) $(15 \mathrm{mg}, 0.075 \mathrm{mmol})$ afforded, after $48 \mathrm{~h}$ of agitation, the title compound (9e) as an off-white powder (145.1 mg, 72\%). $R_{f}=0.50$ (dichloromethane/ethyl acetate 1:1); $[\alpha]^{20}{ }_{\mathrm{D}}-243.0\left(c 0.1, \mathrm{CH}_{2} \mathrm{Cl}_{2}\right) ; \mathrm{HRMS}$ (ESI) $\mathrm{m} / \mathrm{z}$ calcd for $\mathrm{C}_{21} \mathrm{H}_{26} \mathrm{NO}_{7}[\mathrm{M}+\mathrm{H}]^{+} 404.1704$, found 404.1699; ${ }^{1} \mathrm{H} \mathrm{NMR}\left(\mathrm{CDCl}_{3}, 400 \mathrm{MHz}\right): \delta 7.38$ $-7.17(\mathrm{~m}, 5 \mathrm{H}, \mathrm{ArH}), 6.95(\mathrm{~d}, 2 \mathrm{H}, J=9.0 \mathrm{~Hz}, \mathrm{ArH}), 6.79$ (d, 2H, J=9.0 Hz, ArH), 5.79 (t, J=5.8 Hz, $1 \mathrm{H}, \mathrm{NH}), 5.32(\mathrm{~s}, 1 \mathrm{H}, H-1), 5.10(\mathrm{dd}, 1 \mathrm{H}, J=9.7 \mathrm{~Hz}, J=3.1 \mathrm{~Hz}, H-3), 4.43-4.26\left(\mathrm{~m}, 2 \mathrm{H}, \mathrm{ArCH}_{2}\right), 4.23$ - 4.14 (m, 1H, H-2), $3.86-3.72$ (m, 1H, H-5), 3.77 (s, 3H, OCH $), 3.71-3.62$ (m, 1H, H-4), 3.45 (br, $1 \mathrm{H}, \mathrm{OH}), 3.05$ (br, $1 \mathrm{H}, \mathrm{OH}), 1.26(\mathrm{~d}, 3 \mathrm{H}, J=6.1 \mathrm{~Hz}, H-6 \mathrm{a}, H-6 \mathrm{~b}$ and $H-6 \mathrm{c}) .{ }^{13} \mathrm{C} \mathrm{NMR}\left(\mathrm{CDCl}_{3}, 101\right.$ $\mathrm{MHz}): \delta 157.4,155.1,150.2,138.0,128.9,127.8,127.7,117.7,114.7,98.4,75.5,71.7,70.1,69.5,55.8$, 45.4, 17.6. 


\section{References}

(1) Fulmer, G. R.; Miller, A. J. M.; Sherden, N. H.; Gottlieb, H. E.; Nudelman, A.; Stoltz, B. M.; Bercaw, J. E.; Goldberg, K. I., NMR Chemical Shifts of Trace Impurities: Common Laboratory Solvents, Organics, and Gases in Deuterated Solvents Relevant to the Organometallic Chemist. Organometallics 2010, 29, 2176-2179.

(2) Lee, D.; Taylor, M. S., Borinic Acid-Catalyzed Regioselective Acylation of Carbohydrate Derivatives. J. Am. Chem. Soc. 2011, 133, 3724-3727.

(3) Lv, J.; Luo, T.; Zou, D.; Dong, H., Using DMF as Both a Catalyst and Cosolvent for the Regioselective Silylation of Polyols and Diols. Eur. J. Org. Chem. 2019, 6383-6395.

(4) Kumar, A.; Geng, Y.; Schmidt, R. R., Silicon Fluorides for Acid-Base Catalysis in Glycosidations. Adv. Synth. Catal. 2012, 354, 1489-1499.

(5) Padiya, K. J.; Gavade, S.; Kardile, B.; Tiwari, M.; Bajare, S.; Mane, M.; Gaware, V.; Varghese, S.; Harel, D.; Kurhade, S., Unprecedented "In Water" Imidazole Carbonylation: Paradigm Shift for Preparation of Urea and Carbamate. Org. Lett. 2012, 14, 2814-2817.

(6) Dimitrijević, E.; Taylor, M. S., 9-Hetero-10-boraanthracene-derived borinic acid catalysts for regioselective activation of polyols. Chem. Sci. 2013, 4, 3298-3303.

(7) Duspara, P. A.; Islam, M. S.; Lough, A. J.; Batey, R. A., Synthesis and Reactivity of N-Alkyl Carbamoylimidazoles: Development of $N$-Methyl Carbamoylimidazole as a Methyl Isocyanate Equivalent. J. Org. Chem. 2012, 77, 10362-10368.

(8) Mancini, R. S.; McClary, C. A.; Anthonipillai, S.; Taylor, M. S., Organoboron-Promoted Regioselective Glycosylations in the Synthesis of a Saponin-Derived Pentasaccharide from Spergularia ramosa. J. Org. Chem. 2015, 80, 8501-8510.

(9) Agnihotri, G.; Mandal, P. K.; Misra, A. K., Concise synthesis of two trisaccharide analogs related to the glycone constituent of phanoside, a novel insulin releasing natural product. Tetrahedron 2007, 63, 7240-7245.

(10) Elsaidi, H. R. H.; Lowary, T. L., Effect of phenolic glycolipids from Mycobacterium kansasii on proinflammatory cytokine release. A structure-activity relationship study. Chem. Sci. 2015, 6, 31613172. 
6. ${ }^{1} \mathrm{H}$ and ${ }^{13} \mathrm{C}$ NMR Spectra of compound $\mathbf{1}$

a. ${ }^{1} \mathrm{H}$ NMR spectrum $(400 \mathrm{~Hz})$ of methyl 6-O-(tert-butyldimethyl)silyl- $\alpha$-D-mannopyranoside (1)

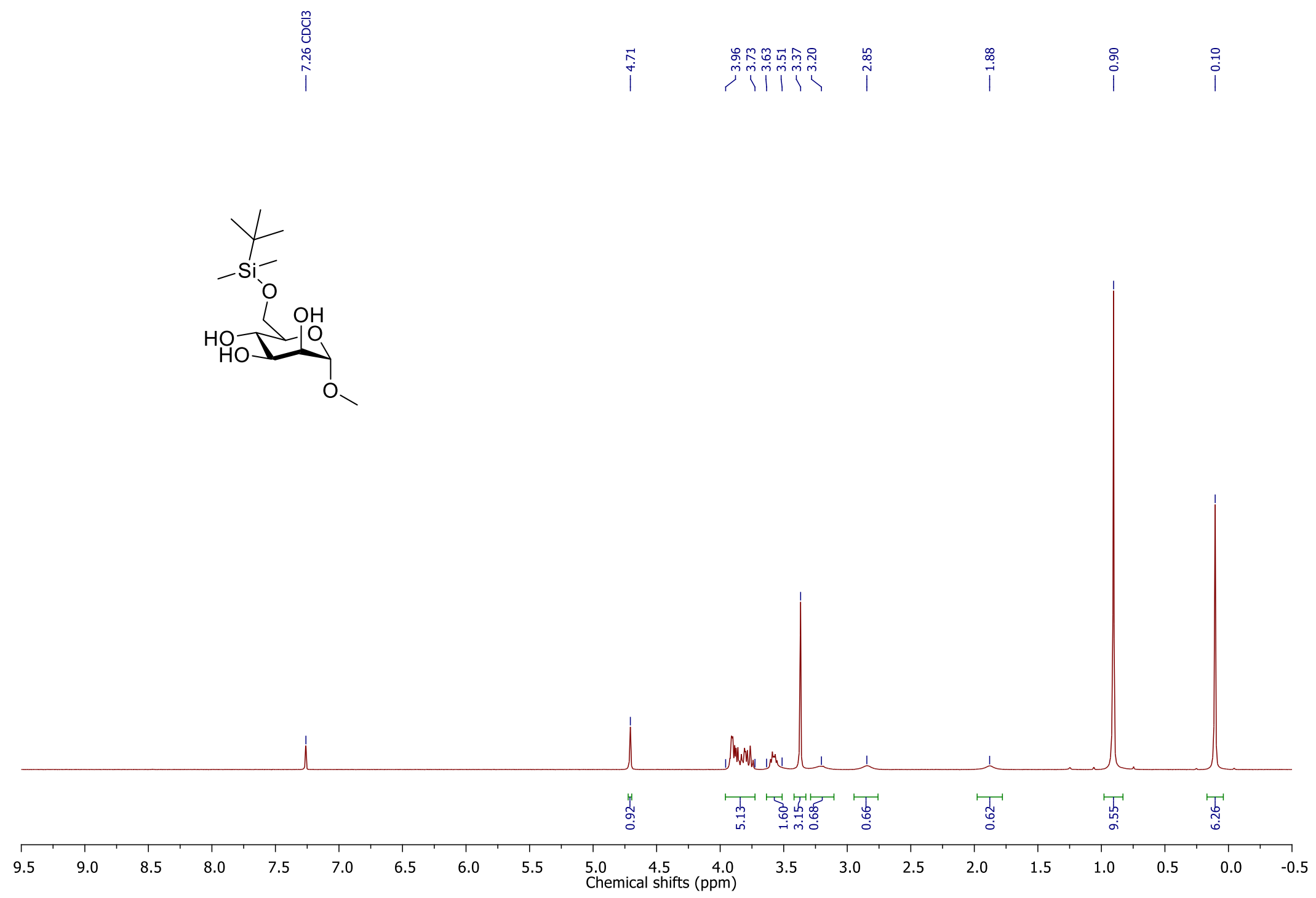


b. ${ }^{13} \mathrm{C}$ NMR spectrum $(101 \mathrm{~Hz})$ of methyl 6-O-(tert-butyldimethyl)silyl- $\alpha$-D-mannopyranoside (1)

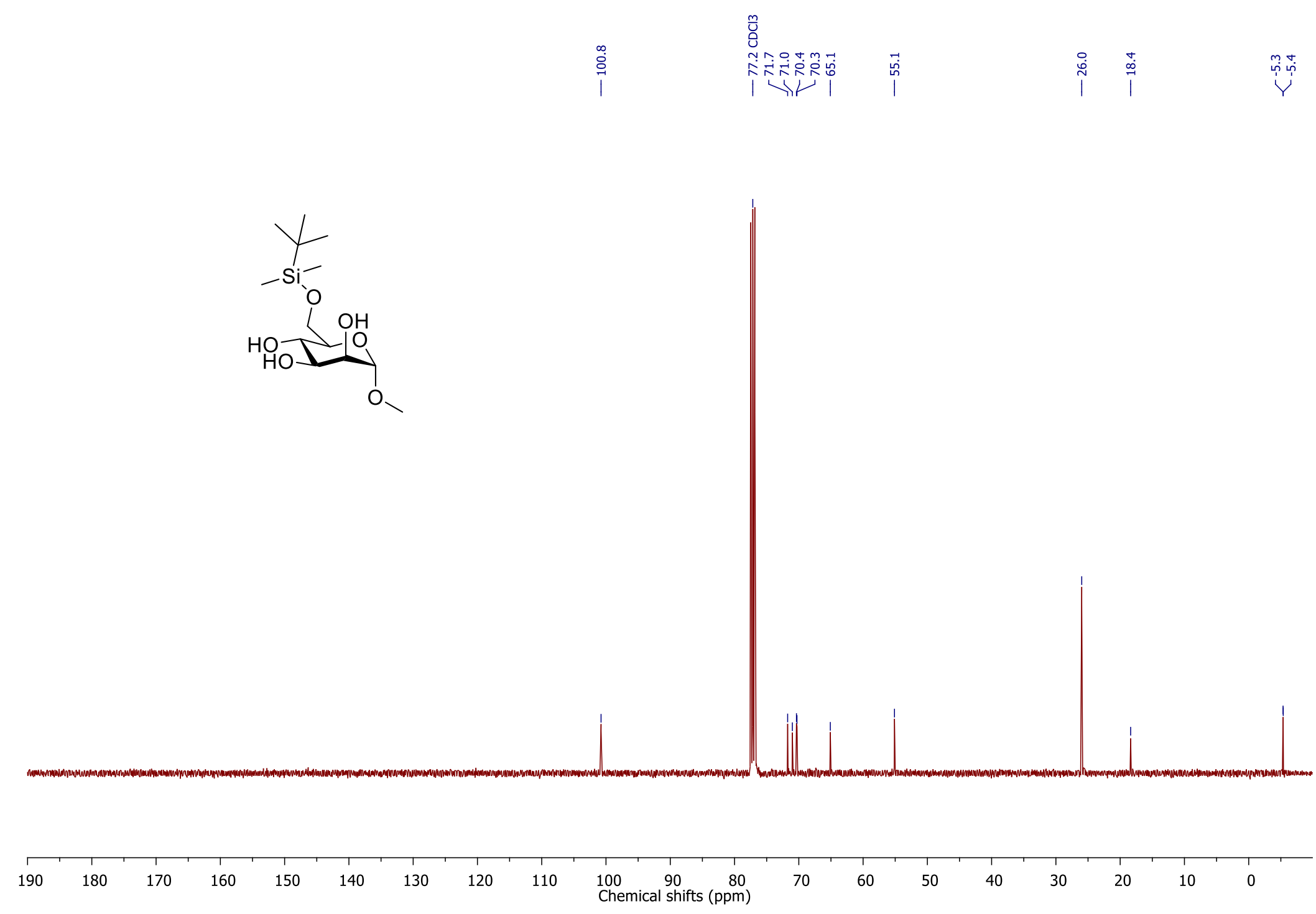


7. ${ }^{1} \mathrm{H}$ and ${ }^{13} \mathrm{C}$ NMR Spectra of compound $\boldsymbol{6 a}$

a. ${ }^{1} \mathrm{H}$ NMR spectrum $(400 \mathrm{~Hz}$ ) of methyl 6-O-(tert-butyldimethyl)silyl- $\beta$-D-galactopyranoside (6a)
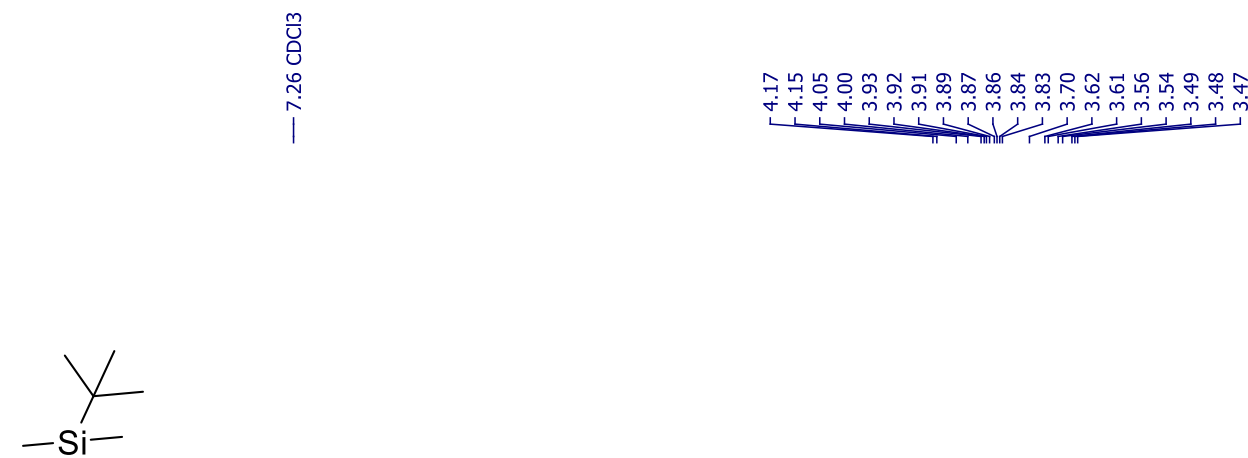

$$
\text { Ho }
$$

$\overbrace{\mathrm{HO}}$

\section{(1)}


b. ${ }^{13} \mathrm{C}$ NMR spectrum $(101 \mathrm{~Hz})$ of methyl 6-O-(tert-butyldimethyl)silyl- $\beta$-D-galactopyranoside (6a)

6a $\left(\mathrm{CDCl}_{3}\right)$

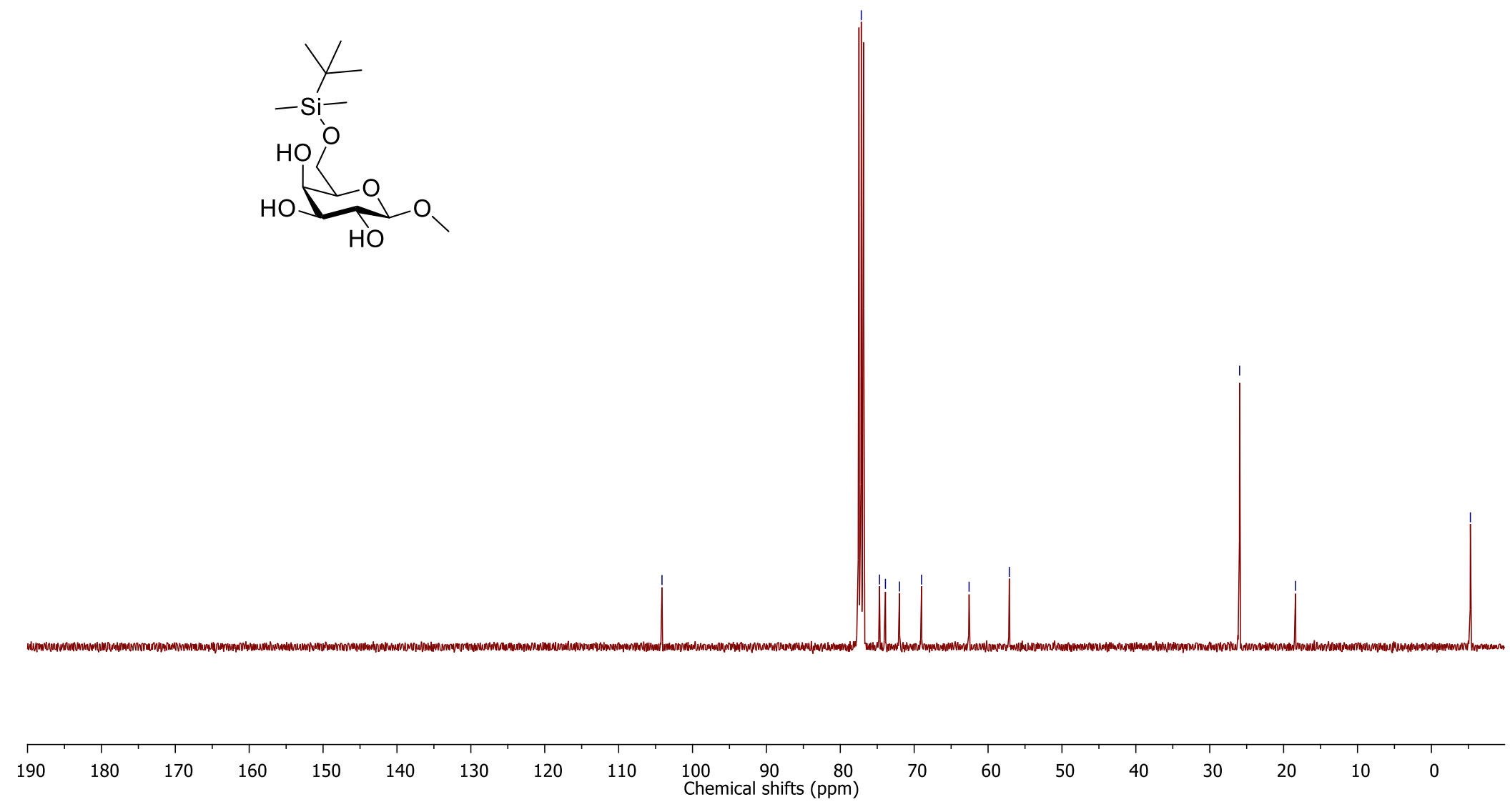

응 
8. ${ }^{1} \mathrm{H}$ and ${ }^{13} \mathrm{C}$ NMR Spectra of compound $\boldsymbol{6 b}$

a. ${ }^{1} \mathrm{H}$ NMR spectrum $(400 \mathrm{~Hz})$ of methyl 6-O-(tert-butyldimethyl)silyl- $\alpha$-D-galactopyranoside (6b) 6b $\left(\mathrm{CDCl}_{3}\right)$
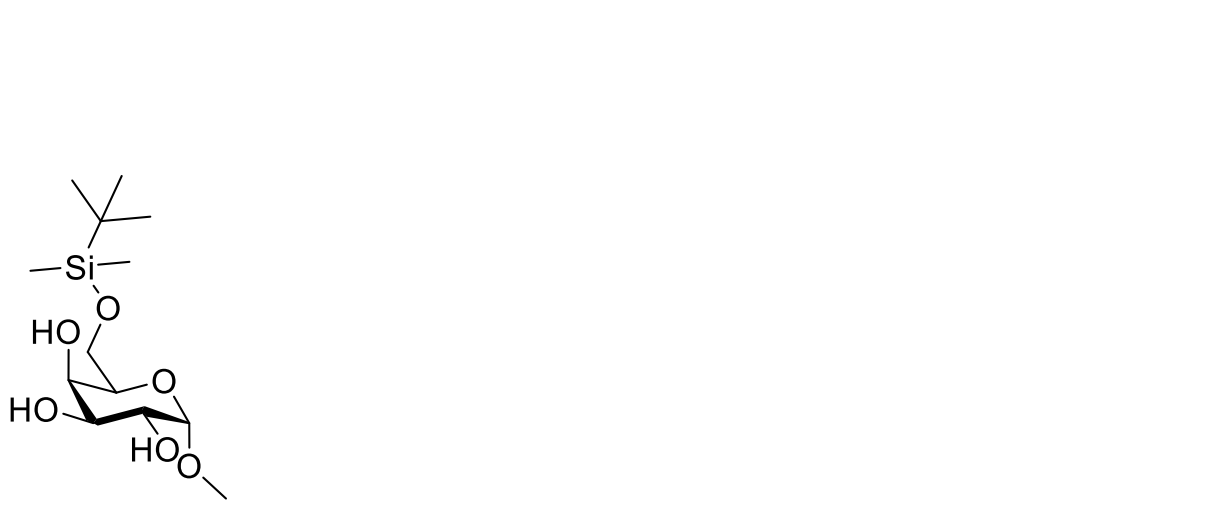
Mu

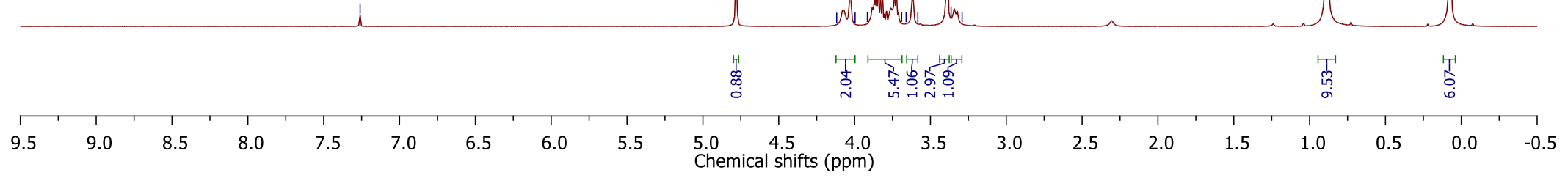


b. ${ }^{13} \mathrm{C}$ NMR spectrum $(101 \mathrm{~Hz})$ of methyl 6-O-(tert-butyldimethyl)silyl- $\alpha$-D-galactopyranoside $(\mathbf{6 b})$

6b $\left(\mathrm{CDCl}_{3}\right)$

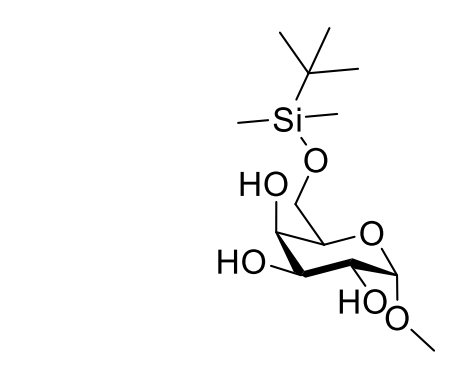

$\stackrel{m}{\circ}$

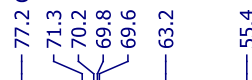

$\stackrel{+}{\stackrel{\infty}{\infty}}$

$\stackrel{m}{\stackrel{m}{p} \overbrace{}^{m}}$

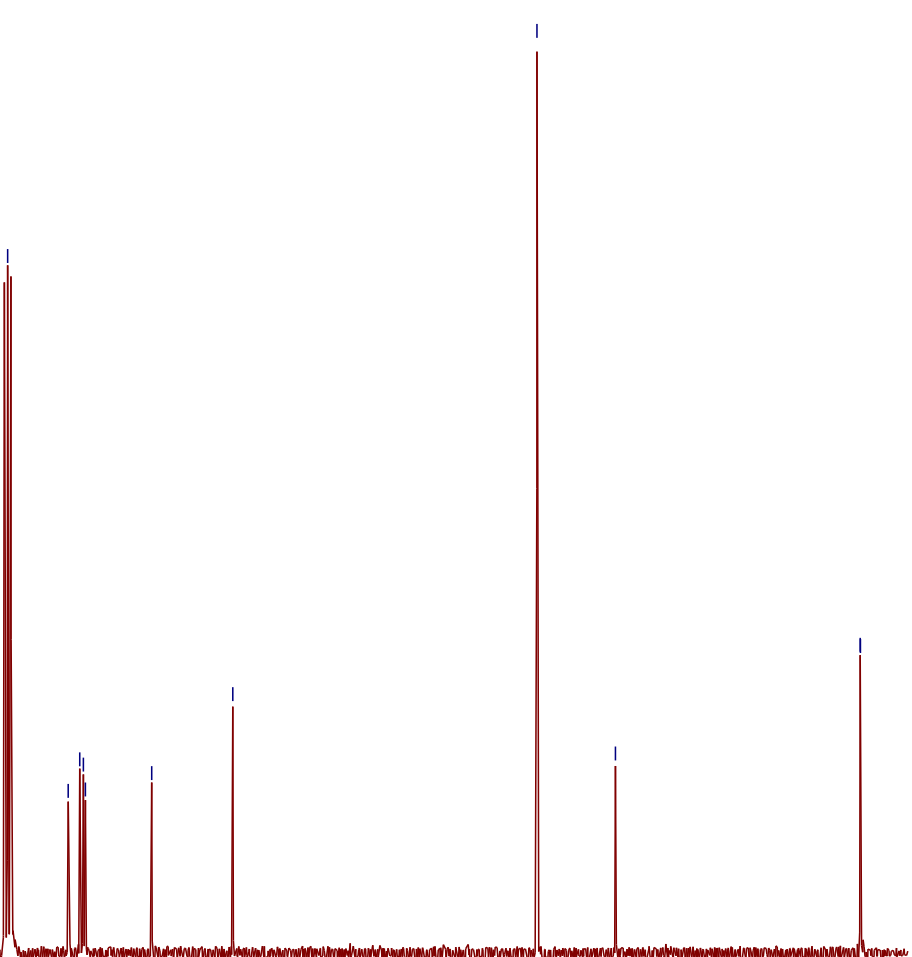


9. ${ }^{1} \mathrm{H}$ and ${ }^{13} \mathrm{C}$ NMR Spectra of compound $8 \mathrm{a}$

a. $\quad{ }^{1} \mathrm{H}$ NMR spectrum $(400 \mathrm{~Hz})$ of 4-methoxyphenyl 6-O-(tert-butyldimethyl)silyl- $\alpha$-D-mannopyranoside (8a)

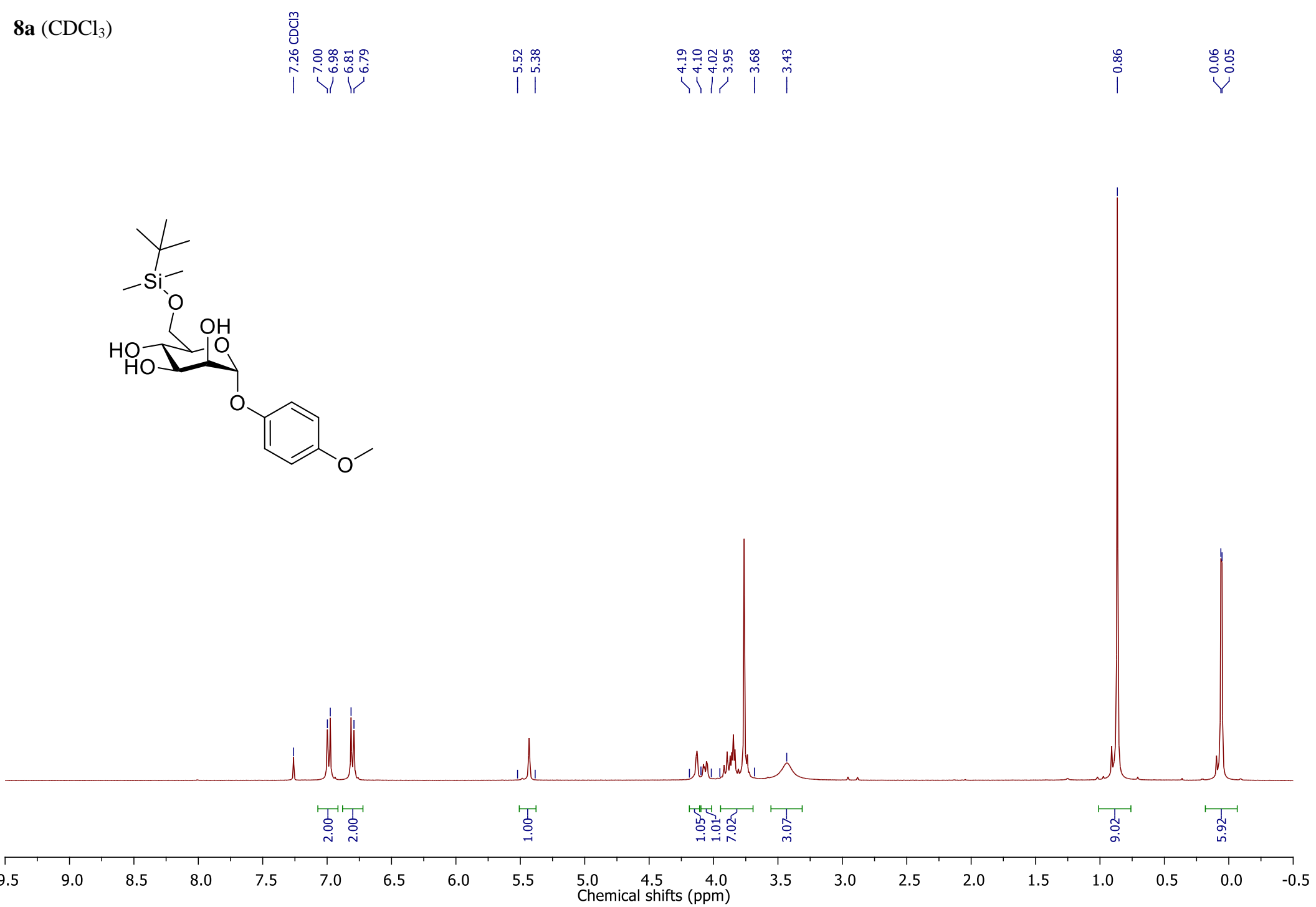


b. ${ }^{13} \mathrm{C}$ NMR spectrum $(101 \mathrm{~Hz})$ of 4-methoxyphenyl 6-O-(tert-butyldimethyl)silyl- $\alpha$-D-mannopyranoside $(\mathbf{8 a})$
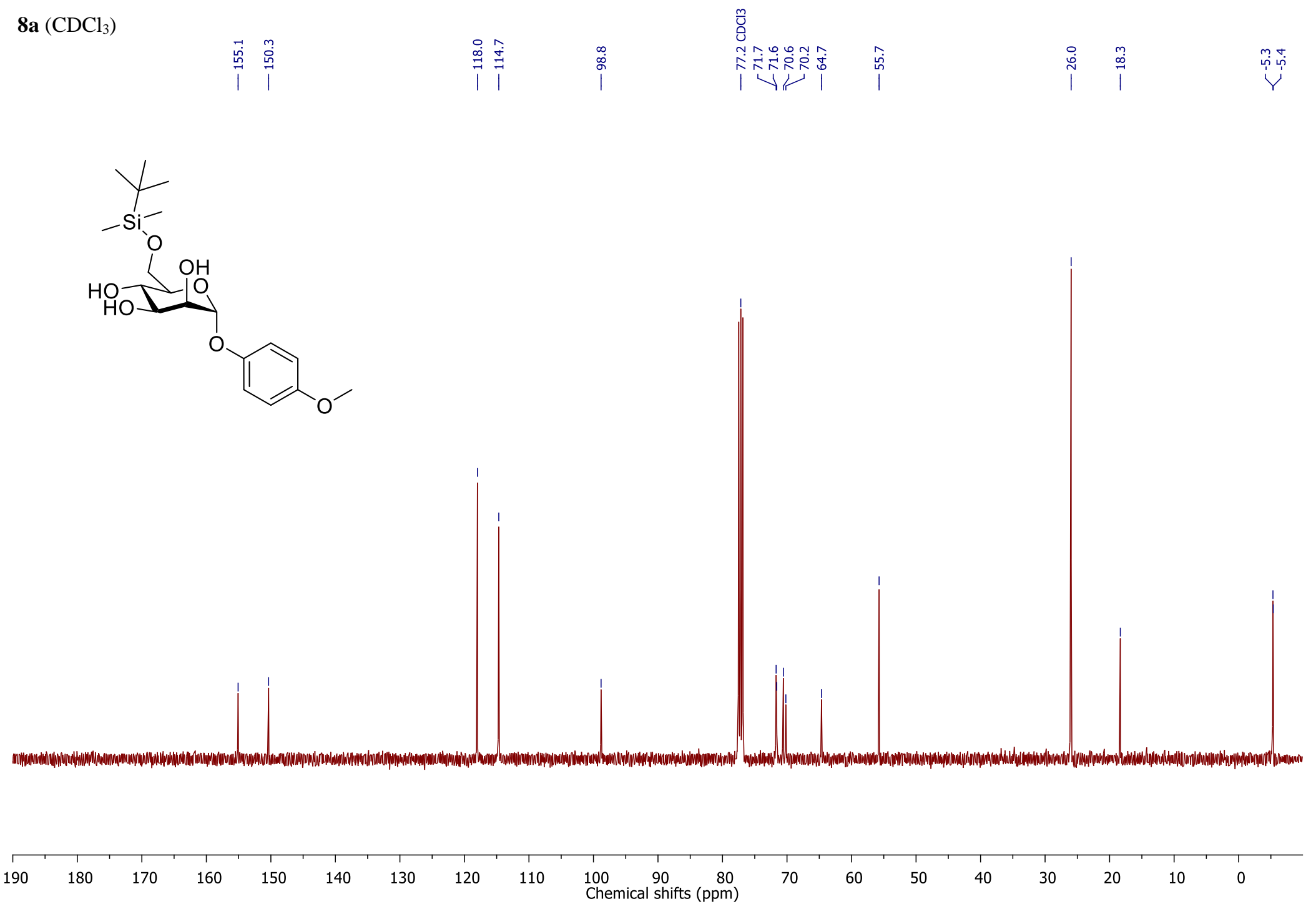
10. ${ }^{1} \mathrm{H}$ and ${ }^{13} \mathrm{C}$ NMR Spectra of compound $8 \boldsymbol{b}$

a. $\quad{ }^{1} \mathrm{H}$ NMR spectrum $(400 \mathrm{~Hz})$ of 4-methoxyphenyl 6-O-(tert-butyldimethyl)silyl- $\beta$-D-galactopyranoside (8b)

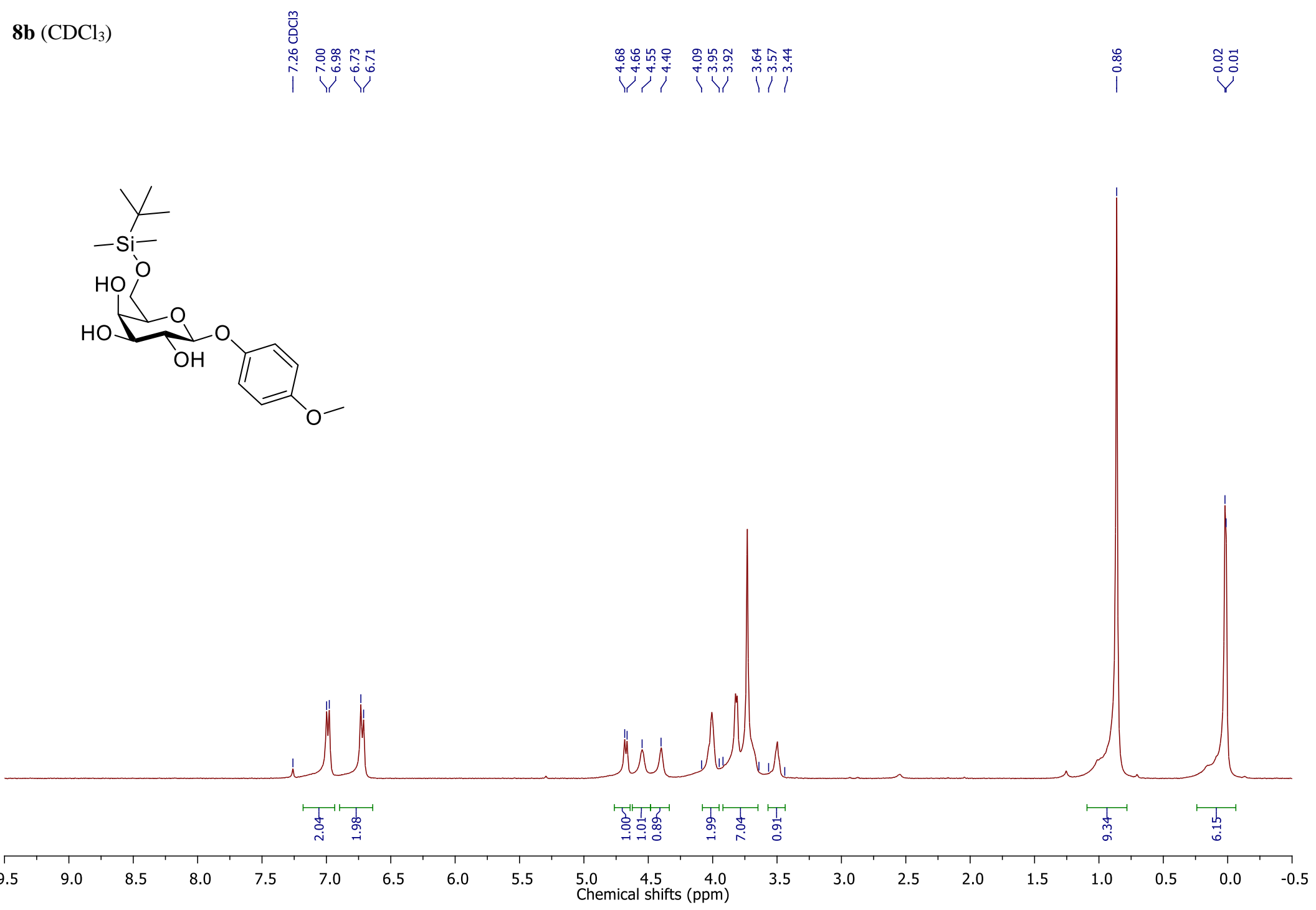


b. ${ }^{13} \mathrm{C}$ NMR spectrum $(101 \mathrm{~Hz})$ of 4-methoxyphenyl 6-O-(tert-butyldimethyl)silyl- $\beta$-D-galactopyranoside (8b)

$\mathbf{8 b}\left(\mathrm{CDCl}_{3}\right)$

总

Y

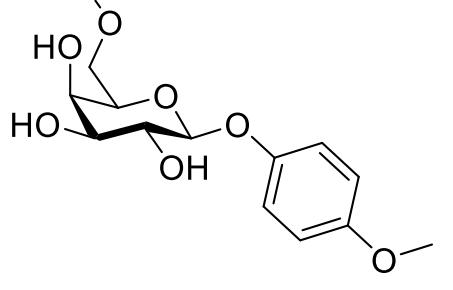

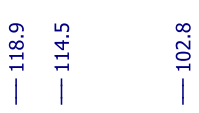

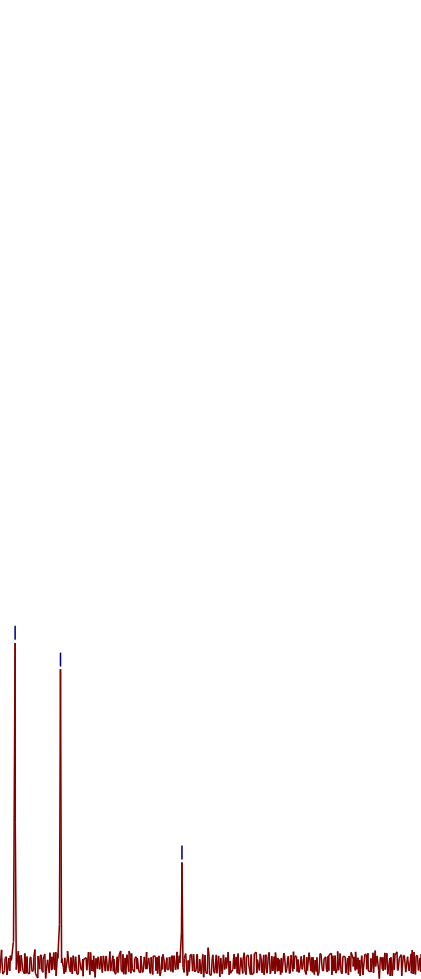

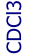

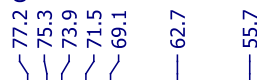

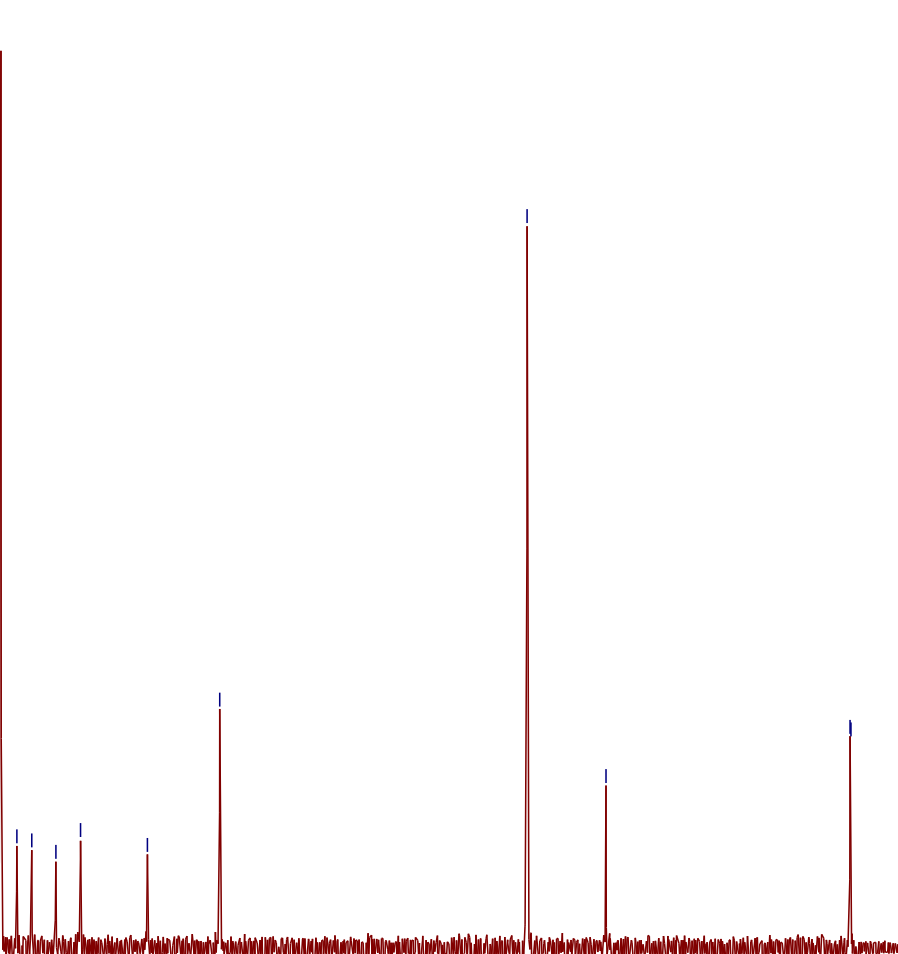

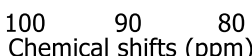

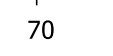

60

50

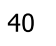

$30 \quad 20$

$20-10$ 
11. ${ }^{1} \mathrm{H}$ and ${ }^{13} \mathrm{C}$ NMR Spectra of compound 10

a. ${ }^{1} \mathrm{H}$ NMR spectrum $(400 \mathrm{~Hz})$ of methyl 6- $O$-(tert-butyldimethyl)silyl- $\alpha$-D-glucopyranoside (10)

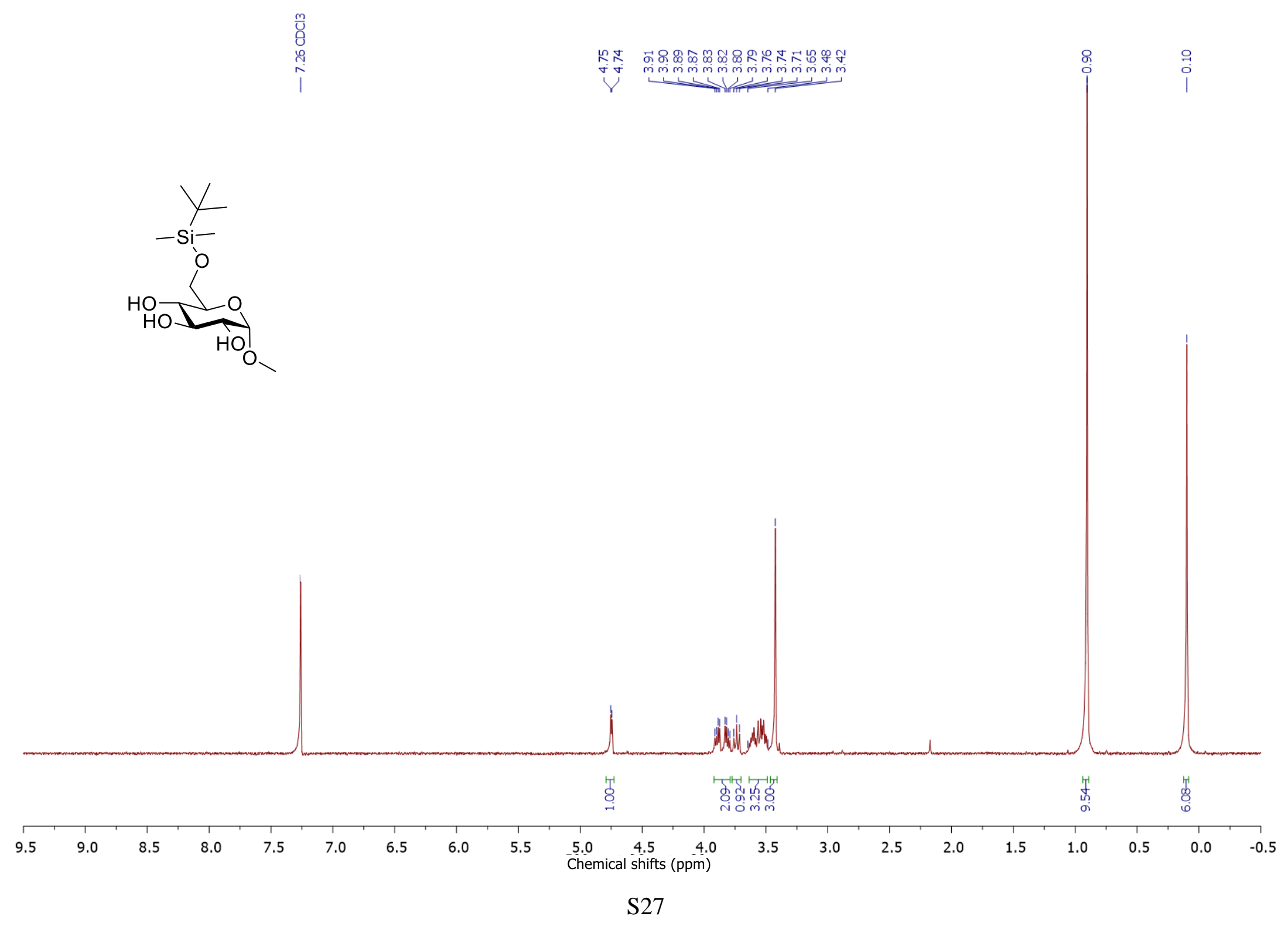


b. ${ }^{13} \mathrm{C}$ NMR spectrum $(101 \mathrm{~Hz})$ of methyl 6-O-(tert-butyldimethyl)silyl- $\alpha$-D-glucopyranoside (10)

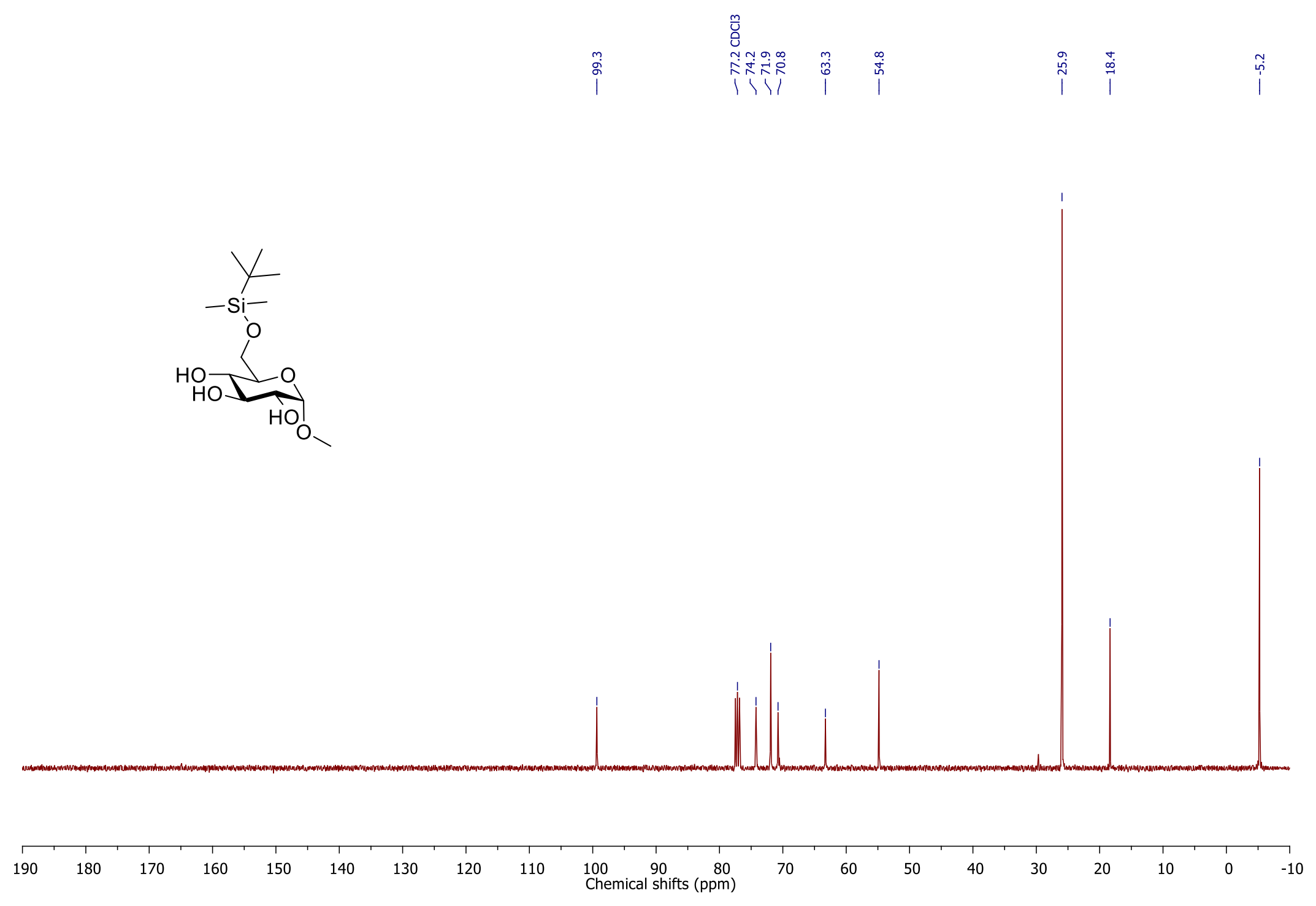




\section{2. ${ }^{1} \mathrm{H}$ and ${ }^{13} \mathrm{C}$ NMR Spectra of compound $2 \mathrm{a}$}

a. $\quad{ }^{1} \mathrm{H}$ NMR spectrum $(400 \mathrm{~Hz})$ of 1-benzyl-carbamoylimidazole (2a)

$$
\text { i }
$$

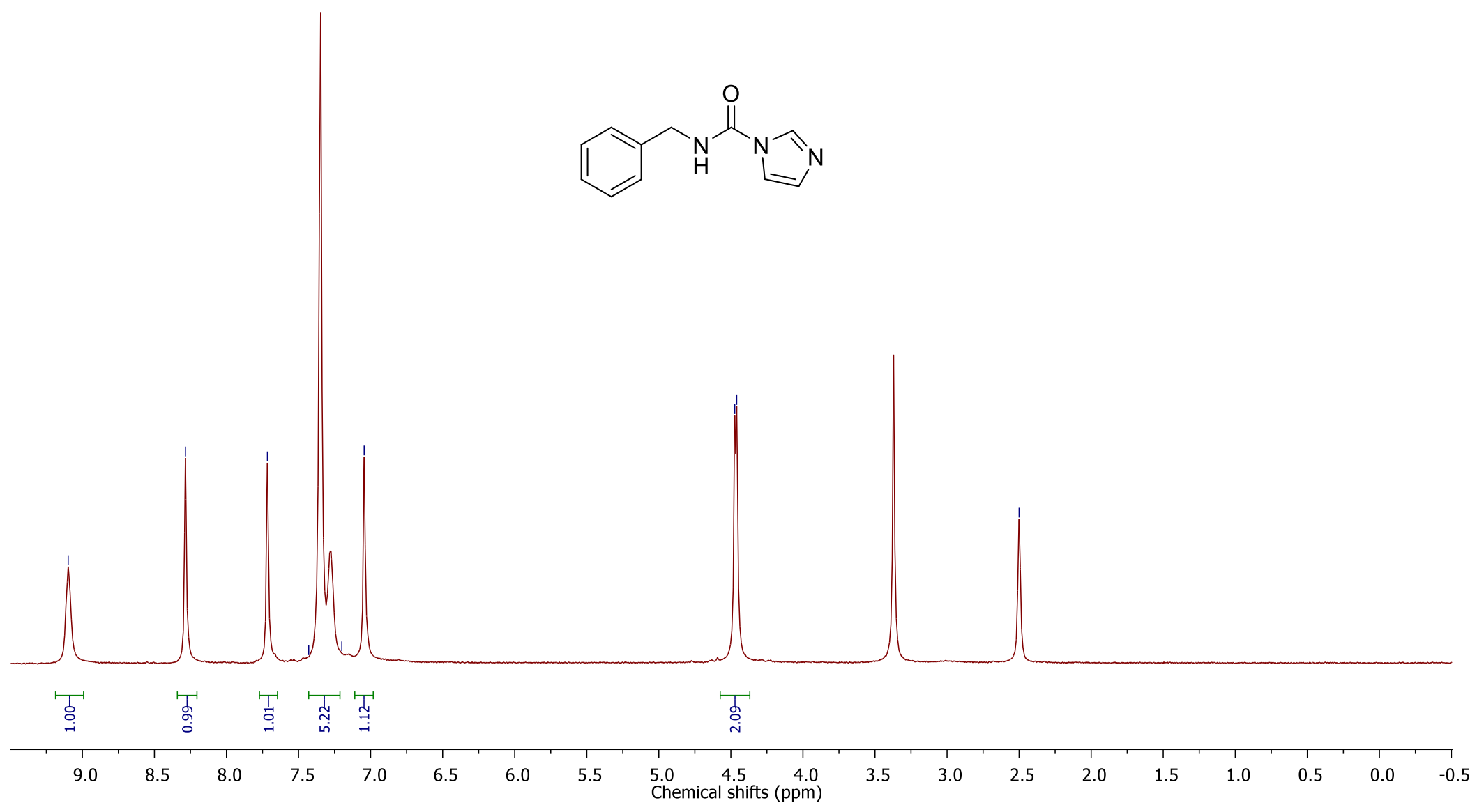


b. ${ }^{13} \mathrm{C}$ NMR spectrum $(101 \mathrm{~Hz})$ of 1-benzyl-carbamoylimidazole (2a)
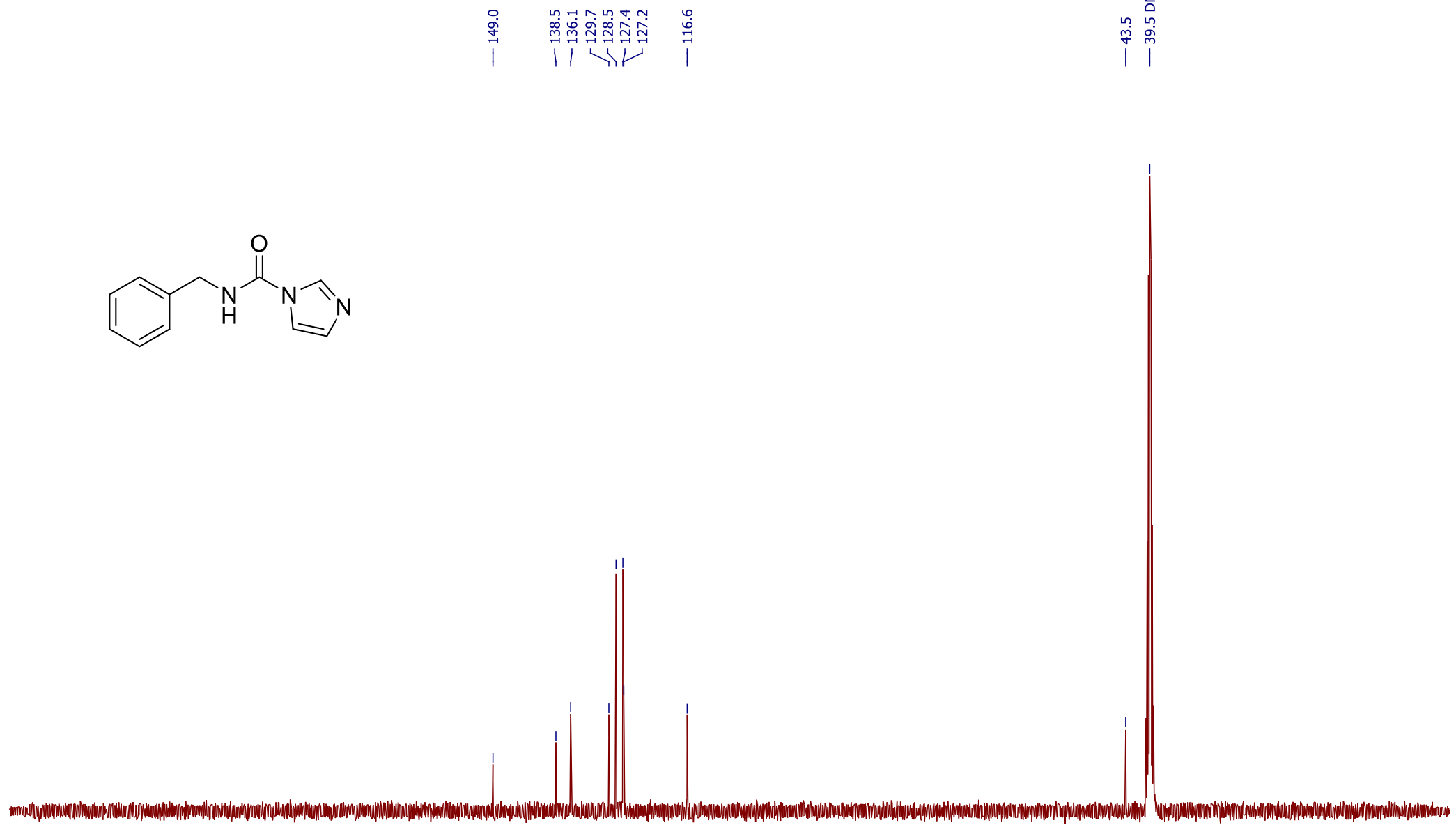


\section{3. ${ }^{1} \mathrm{H}$ and ${ }^{13} \mathrm{C}$ NMR Spectra of compound $2 \mathrm{c}$}

a. $\quad{ }^{1} \mathrm{H}$ NMR spectrum $(400 \mathrm{~Hz})$ of 1-butyl-carbamoylimidazole (2c)

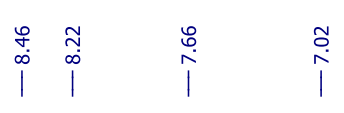

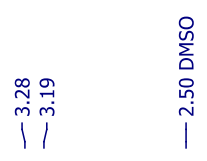
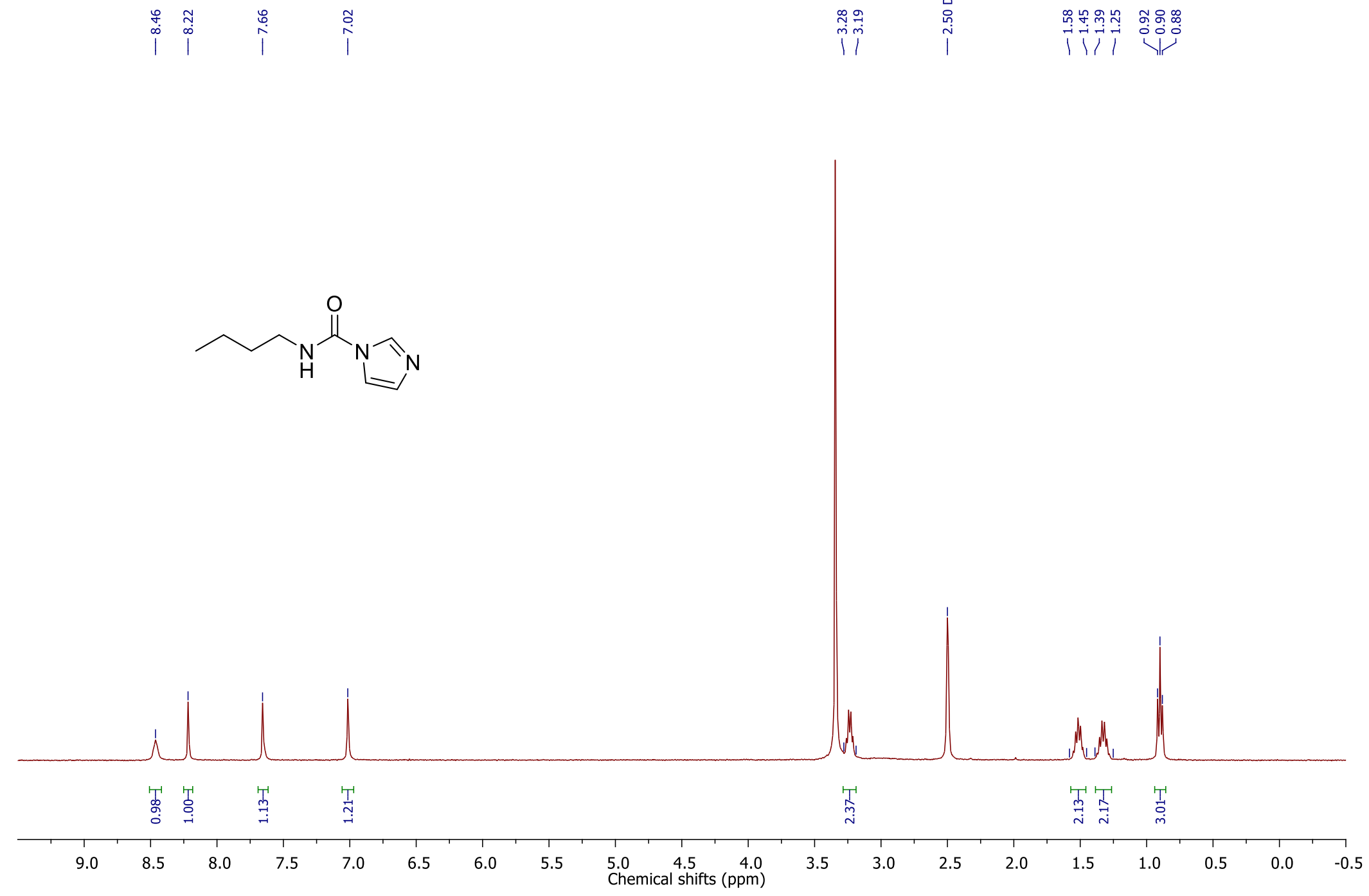
b. ${ }^{13} \mathrm{C}$ NMR spectrum $(101 \mathrm{~Hz})$ of 1-butyl-carbamoylimidazole (2c)

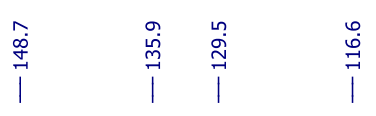

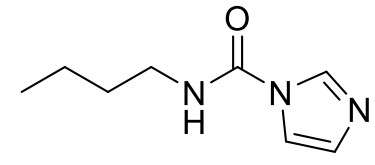




\section{4. ${ }^{1} \mathrm{H}$ and ${ }^{13} \mathrm{C}$ NMR Spectra of compound $2 \mathrm{~d}$}

a. ${ }^{1} \mathrm{H}$ NMR spectrum $(400 \mathrm{~Hz})$ of 1-octyl-carbamoylimidazole (2d)
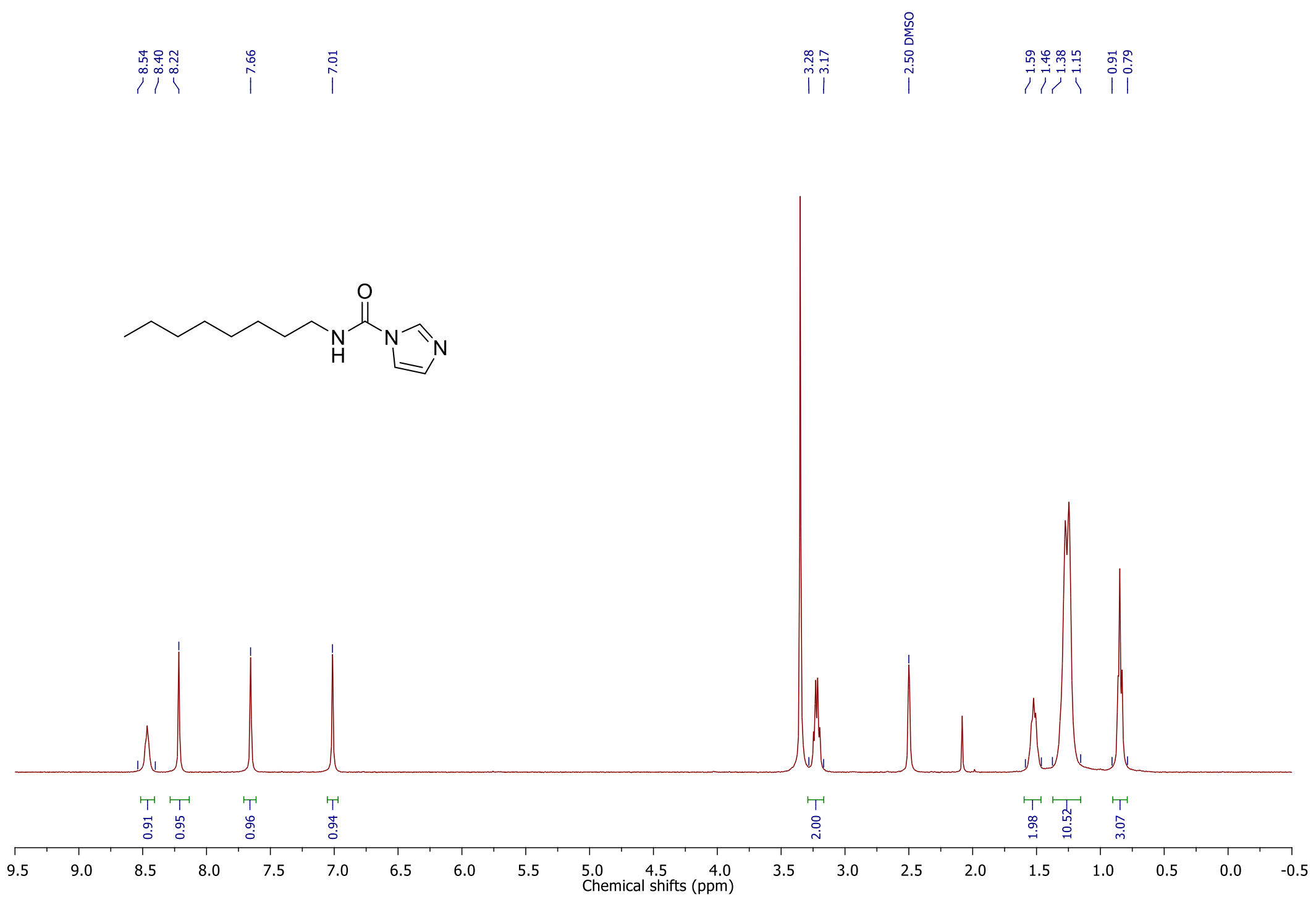
b. ${ }^{13} \mathrm{C}$ NMR spectrum $(101 \mathrm{~Hz})$ of 1-octyl-carbamoylimidazole (2d)

l

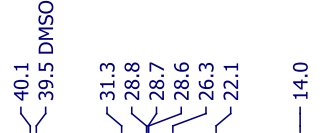
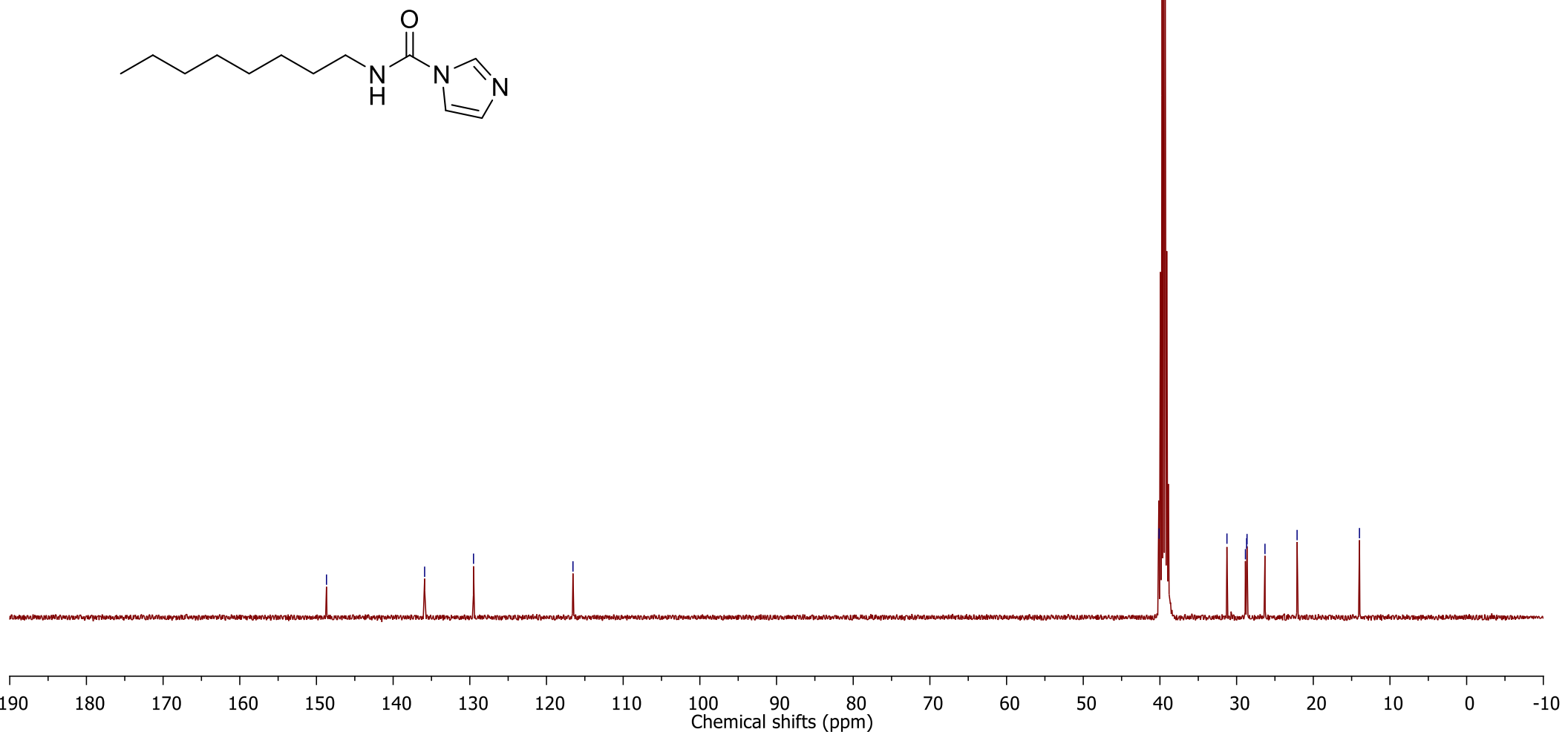


\section{5. ${ }^{1} \mathrm{H}$ and ${ }^{13} \mathrm{C}$ NMR Spectra of compound $2 \mathrm{e}$}

a. ${ }^{1} \mathrm{H}$ NMR spectrum $(400 \mathrm{~Hz})$ of 1-cyclohexyl-carbamoylimidazole (2e)
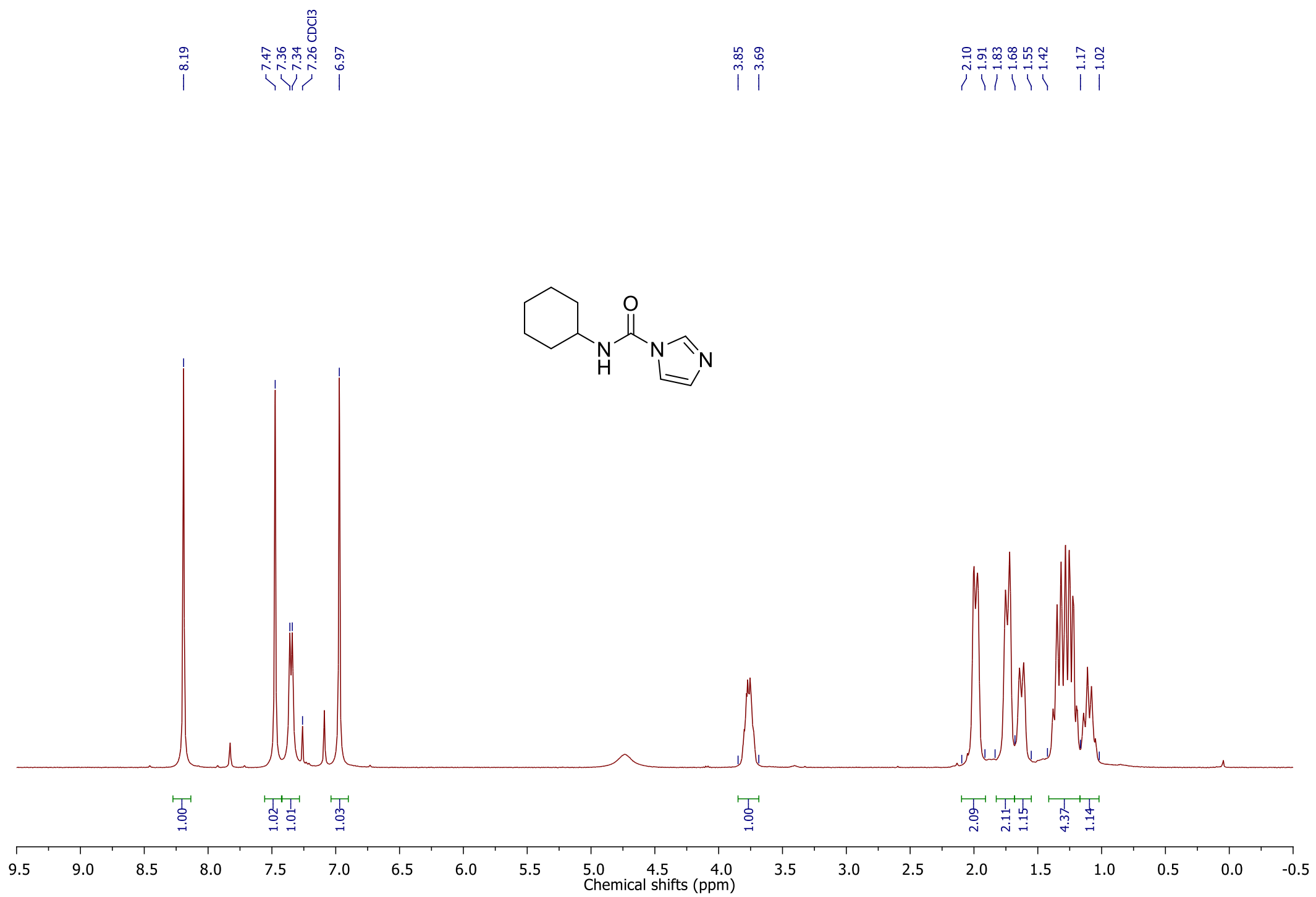
b. ${ }^{13} \mathrm{C}$ NMR spectrum $(101 \mathrm{~Hz})$ of 1-cyclohexyl-carbamoylimidazole (2e)

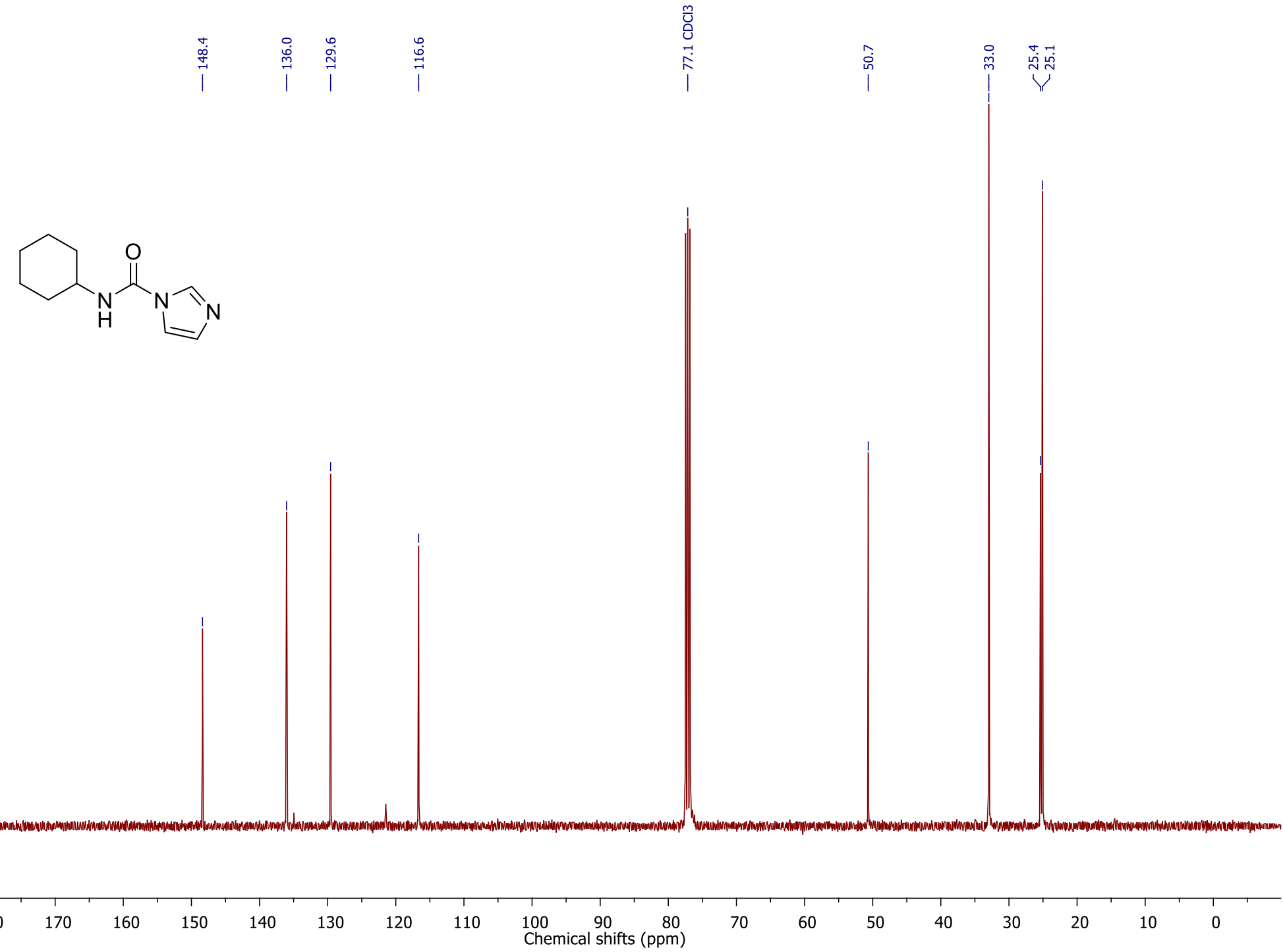


16. ${ }^{1} \mathrm{H}$ and ${ }^{13} \mathrm{C}$ NMR Spectra of compound $2 f$

a. $\quad{ }^{1} \mathrm{H}$ NMR spectrum $(400 \mathrm{~Hz})$ of $(1 H$-imidazol-1-yl)(piperidin-1-yl)methanone (2f)

$$
\text { i. }
$$
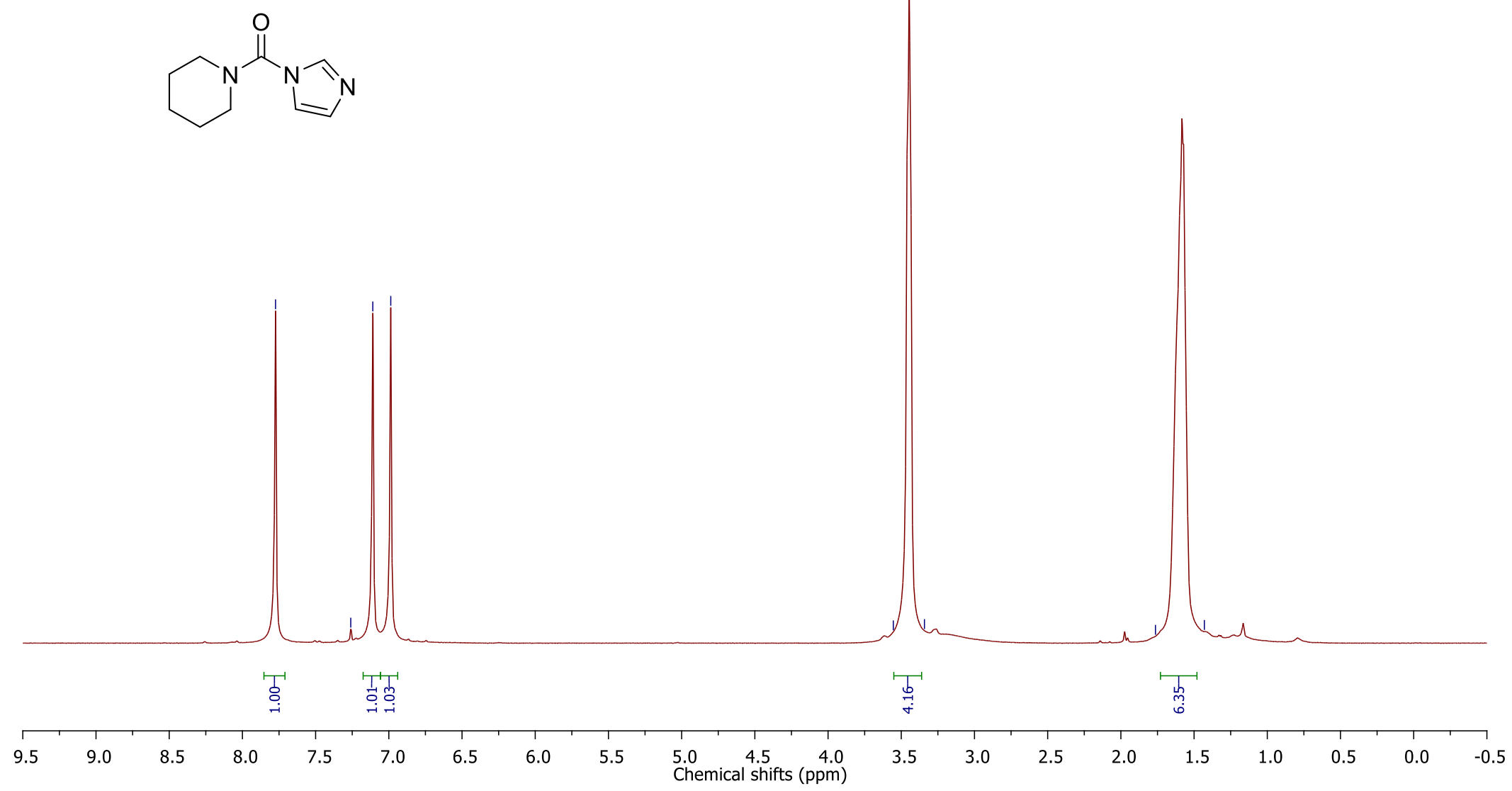
b. ${ }^{13} \mathrm{C}$ NMR spectrum $(101 \mathrm{~Hz})$ of $(1 H$-imidazol-1-yl)(piperidin-1-yl)methanone (2f)

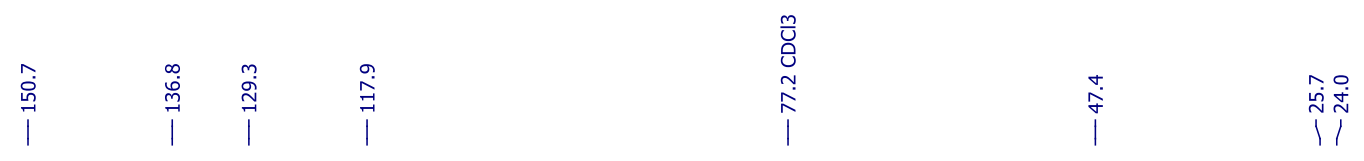<smiles>O=C(N1CCCCC1)n1ccnc1</smiles>

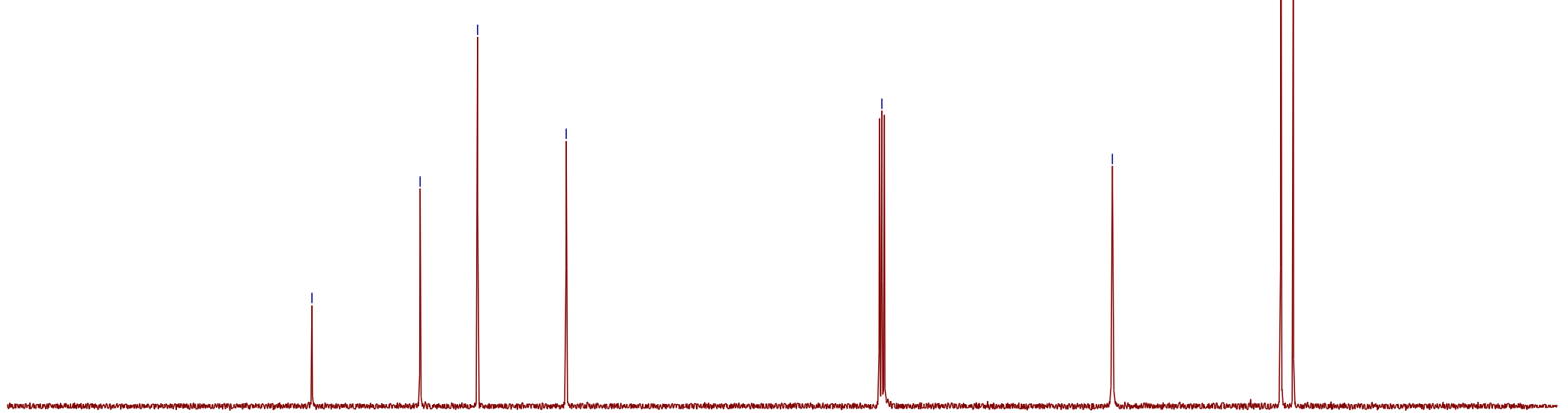

190180

$170 \quad 160$

150

$140 \quad 130$

120 


\section{7. ${ }^{1} \mathrm{H},{ }^{13} \mathrm{C}$, and COSY NMR Spectra of compound $3 a$}

a. $\quad{ }^{1} \mathrm{H}$ NMR spectrum $(400 \mathrm{~Hz}$ ) of methyl 3-O-benzylcarbamoyl-6-O-(tert-butyldimethyl)silyl- $\alpha$-D-mannopyranoside (3a)

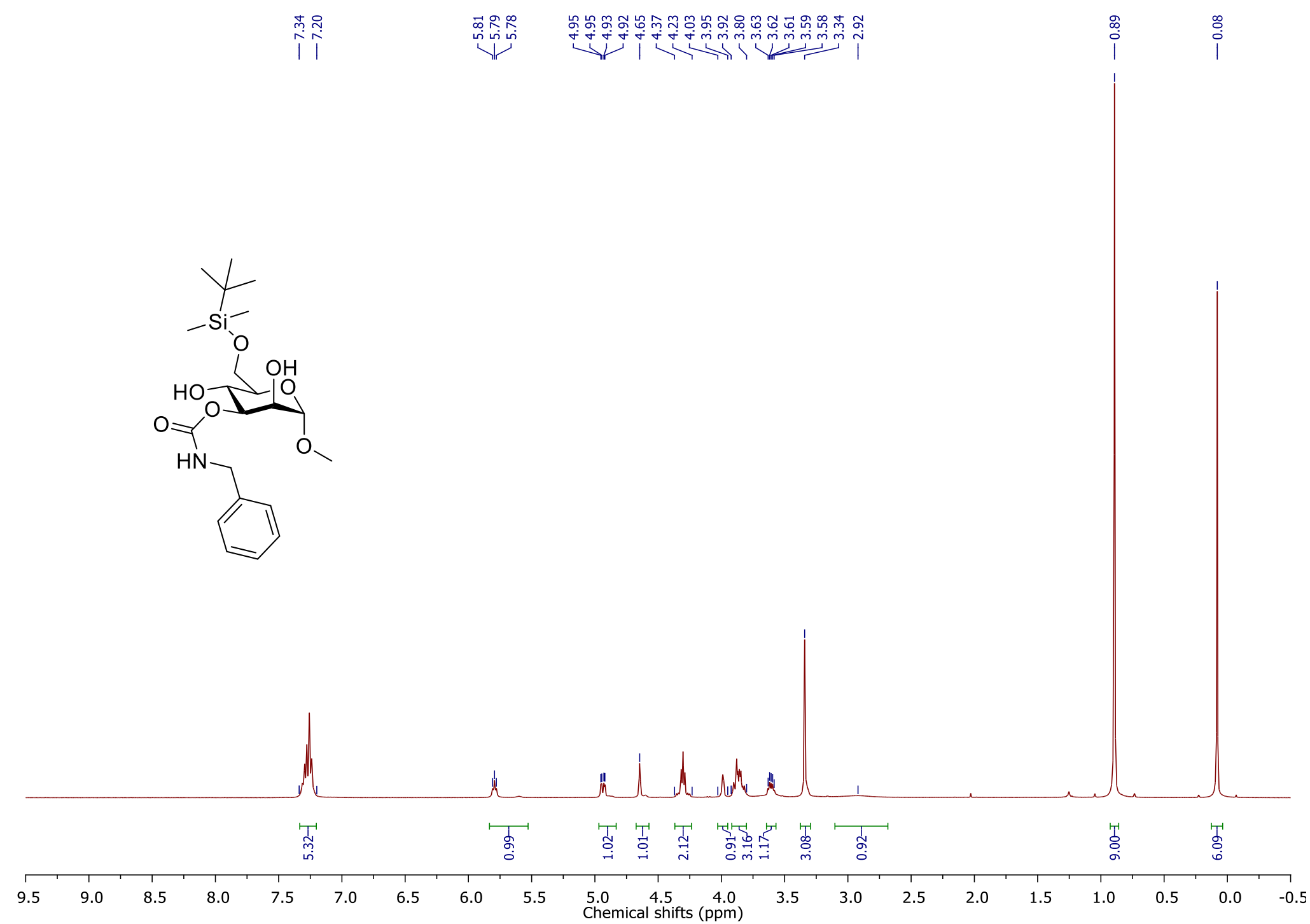


b. ${ }^{13} \mathrm{C}$ NMR spectrum $(101 \mathrm{~Hz})$ of methyl 3-O-benzylcarbamoyl-6-O-(tert-butyldimethyl)silyl- $\alpha$-D-mannopyranoside (3a)
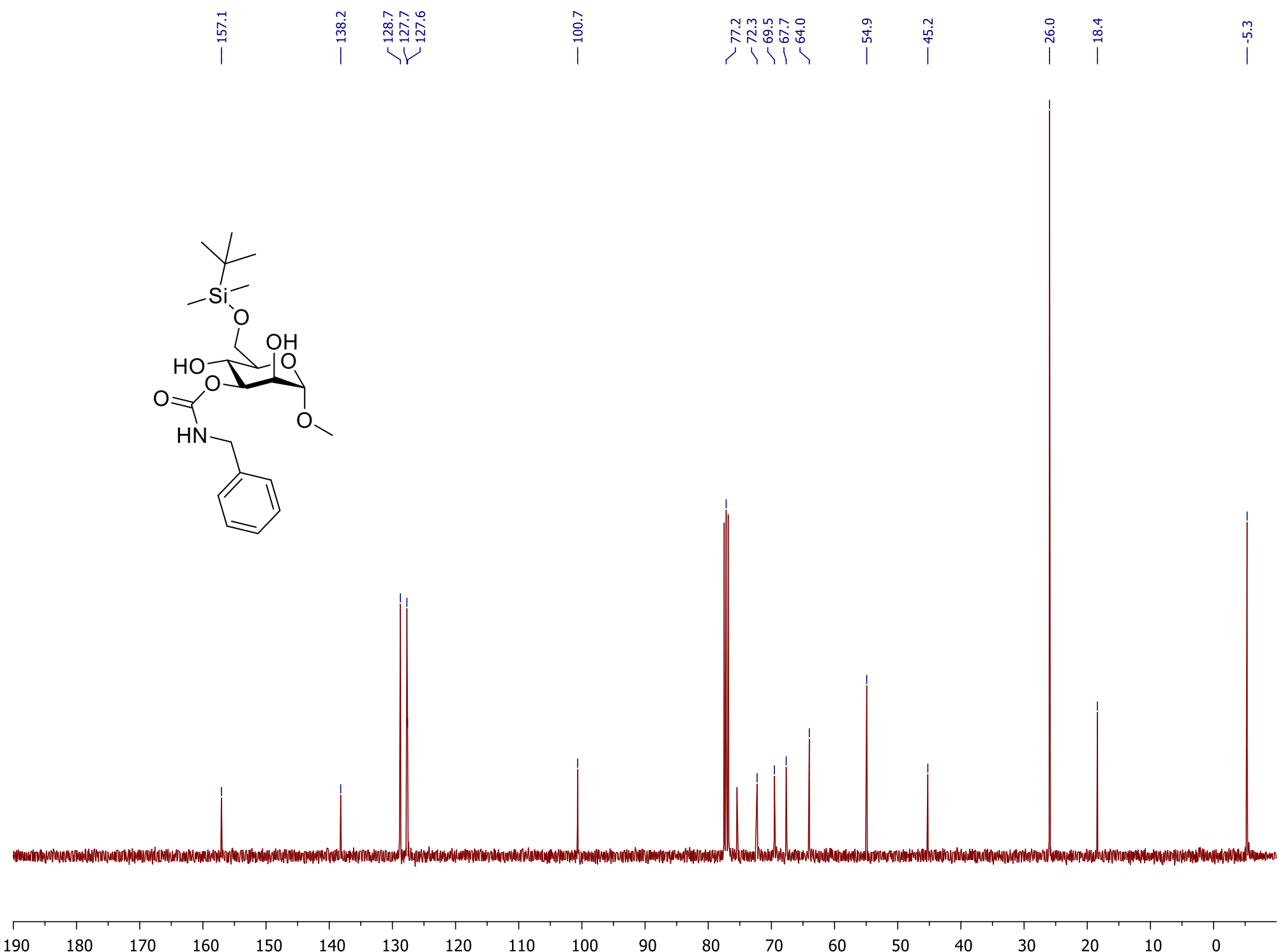

170

160

150

140

130

120

$10 \quad 100$

$\begin{array}{llc}00 & 90 & 80 \\ \text { Chemical } & \text { shifts } & (\mathrm{ppm})\end{array}$

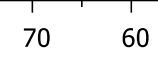

50
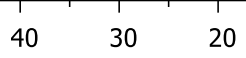

10 
c. COSY NMR spectrum $(400 \mathrm{~Hz}$ ) of methyl 3-O-benzylcarbamoyl-6-O-(tert-butyldimethyl)silyl- $\alpha$-D-mannopyranoside (3a)

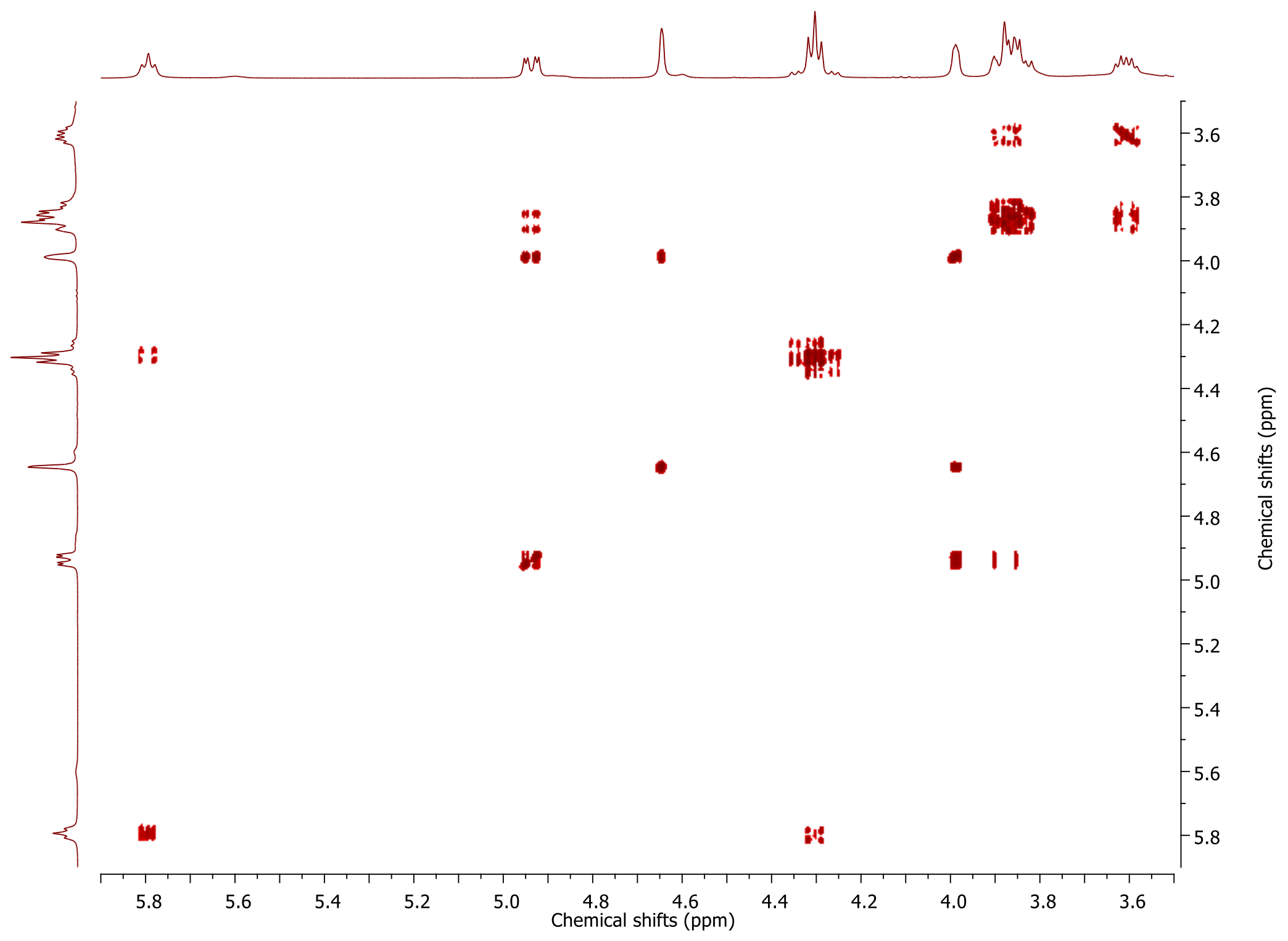


18. ${ }^{1} \mathrm{H},{ }^{13} \mathrm{C}$, and COSY NMR Spectra of compound $\mathbf{3 b}$

a. ${ }^{1} \mathrm{H}$ NMR spectrum $(400 \mathrm{~Hz}$ ) of methyl 3-O-methylcarbamoyl-6- $O$-(tert-butyldimethyl)silyl- $\alpha$-D-mannopyranoside (3b)
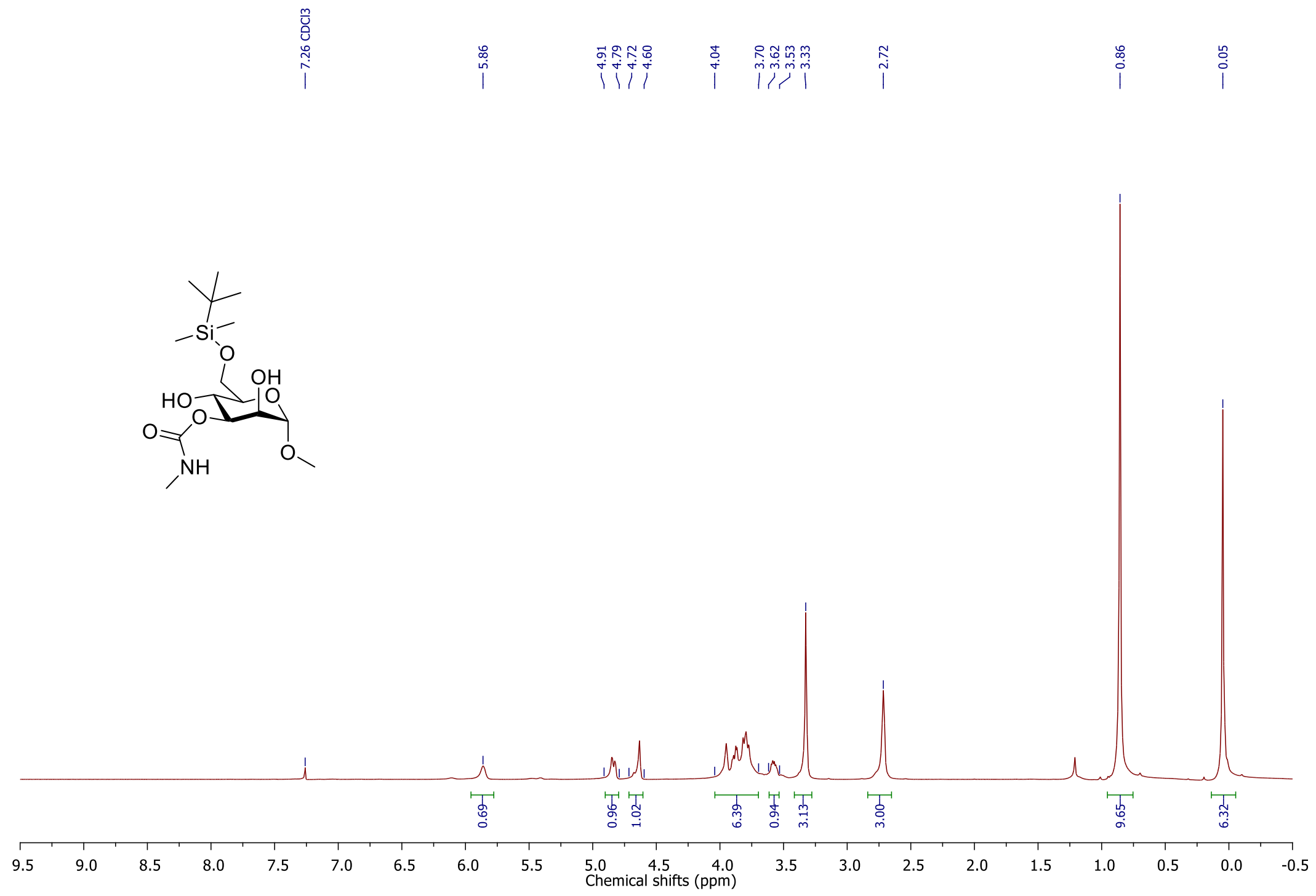
b. ${ }^{13} \mathrm{C}$ NMR spectrum $(101 \mathrm{~Hz})$ of methyl 3-O-methylcarbamoyl-6-O-(tert-butyldimethyl)silyl- $\alpha$-D-mannopyranoside (3b)

$\mathbf{3 b}\left(\mathrm{CDCl}_{3}\right)$

$\stackrel{\hat{\tilde{n}}}{\stackrel{0}{\mid}}$
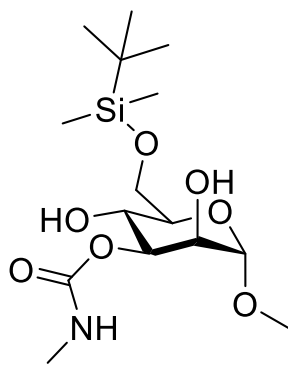

商

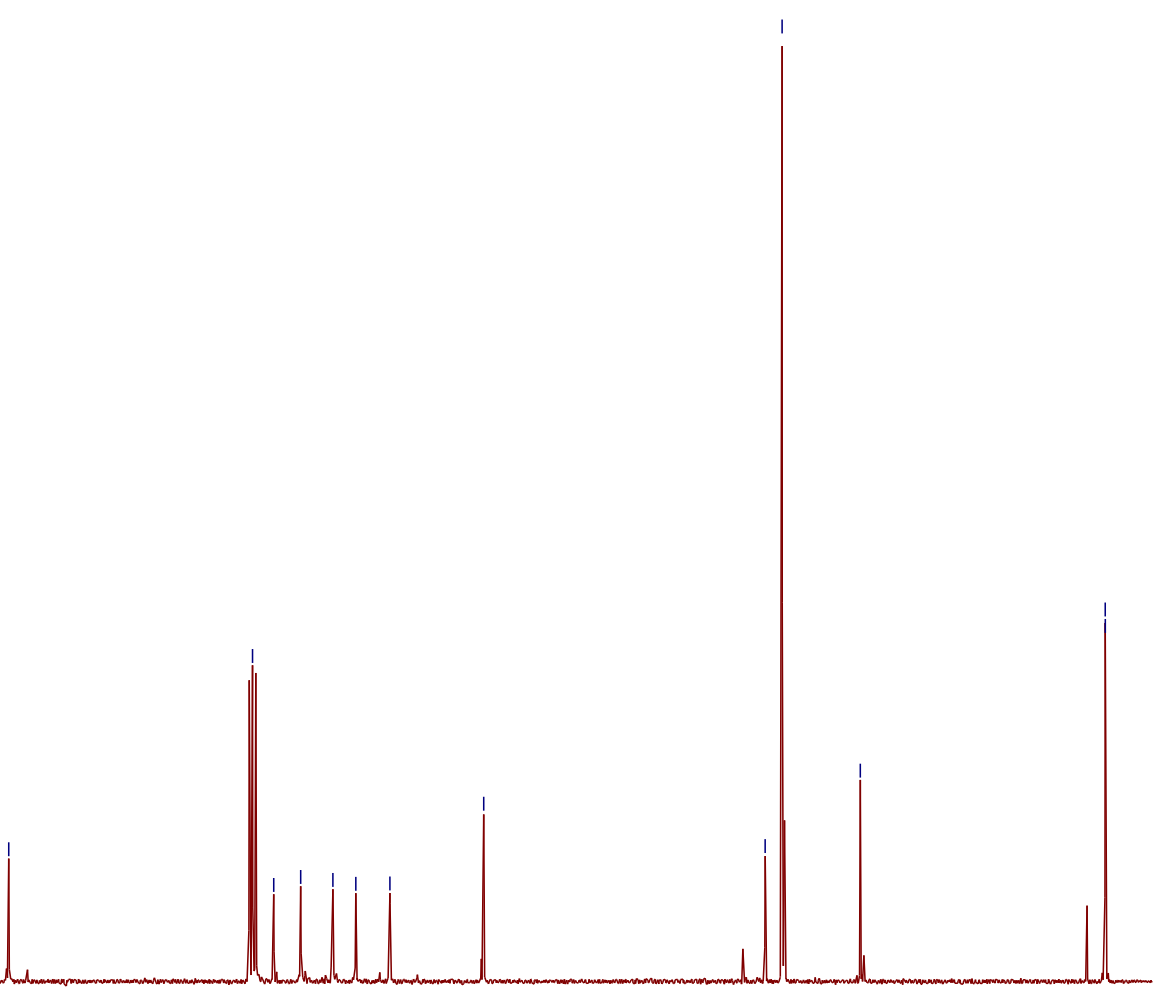

190

180

170

160

150

140

$130 \quad 120$

$110 \quad 100$

$\begin{array}{llc}100 & 90 & 80 \\ \text { Chemical } & \text { shifts } & (\mathrm{ppm})\end{array}$ 
c. COSY NMR spectrum (400 Hz) of methyl 3-O-methylcarbamoyl-6- $O$-(tert-butyldimethyl)silyl- $\alpha$-D-mannopyranoside (3b)

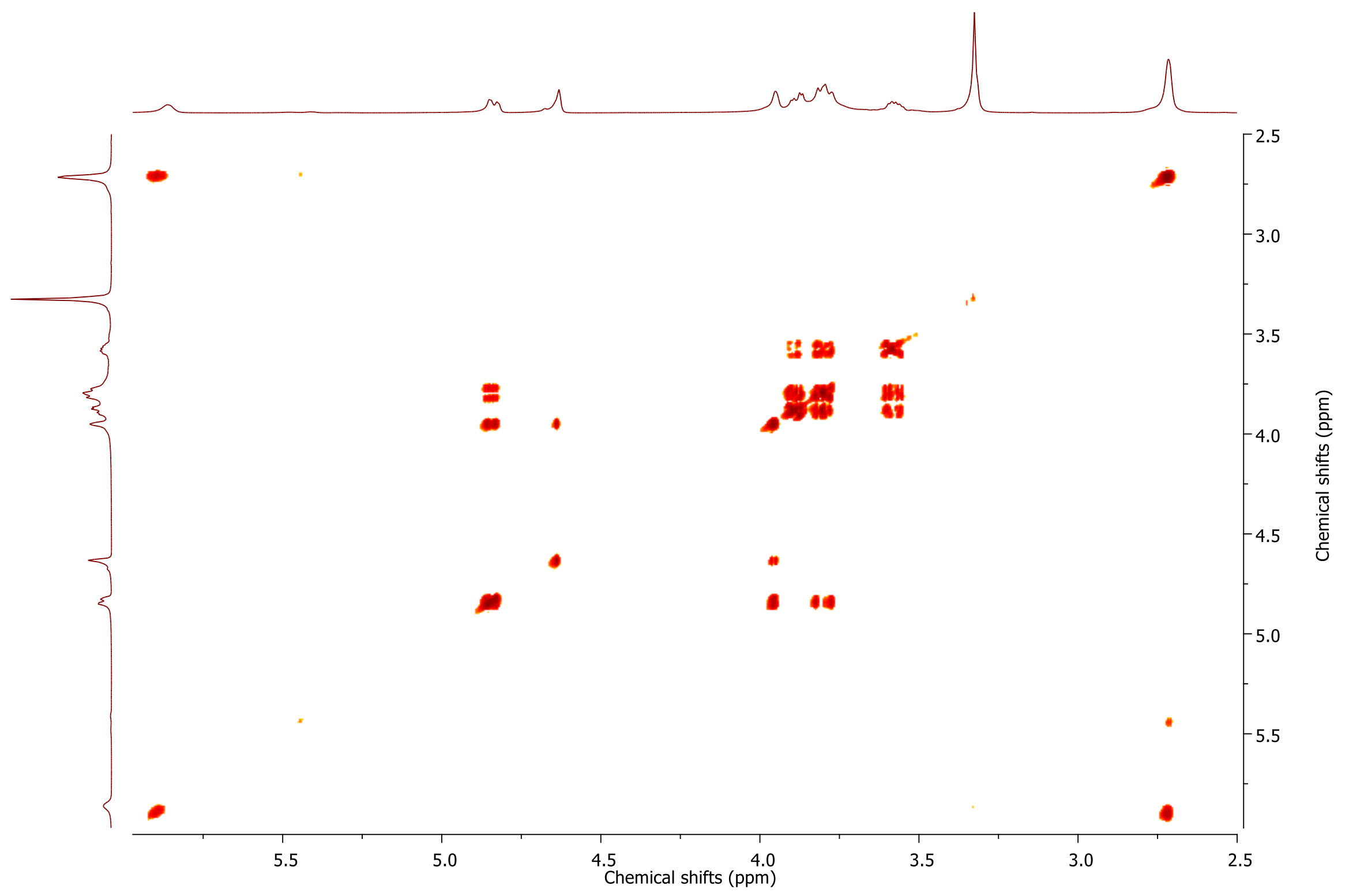


19. ${ }^{1} \mathrm{H},{ }^{13} \mathrm{C}$, and COSY NMR Spectra of compound $3 \mathrm{c}$

a. $\quad{ }^{1} \mathrm{H}$ NMR spectrum $(400 \mathrm{~Hz})$ of methyl 3-O-butylcarbamoyl-6-O-(tert-butyldimethyl)silyl- $\alpha$-D-mannopyranoside (3c) $3 \mathbf{c}\left(\mathrm{CDCl}_{3}\right)$
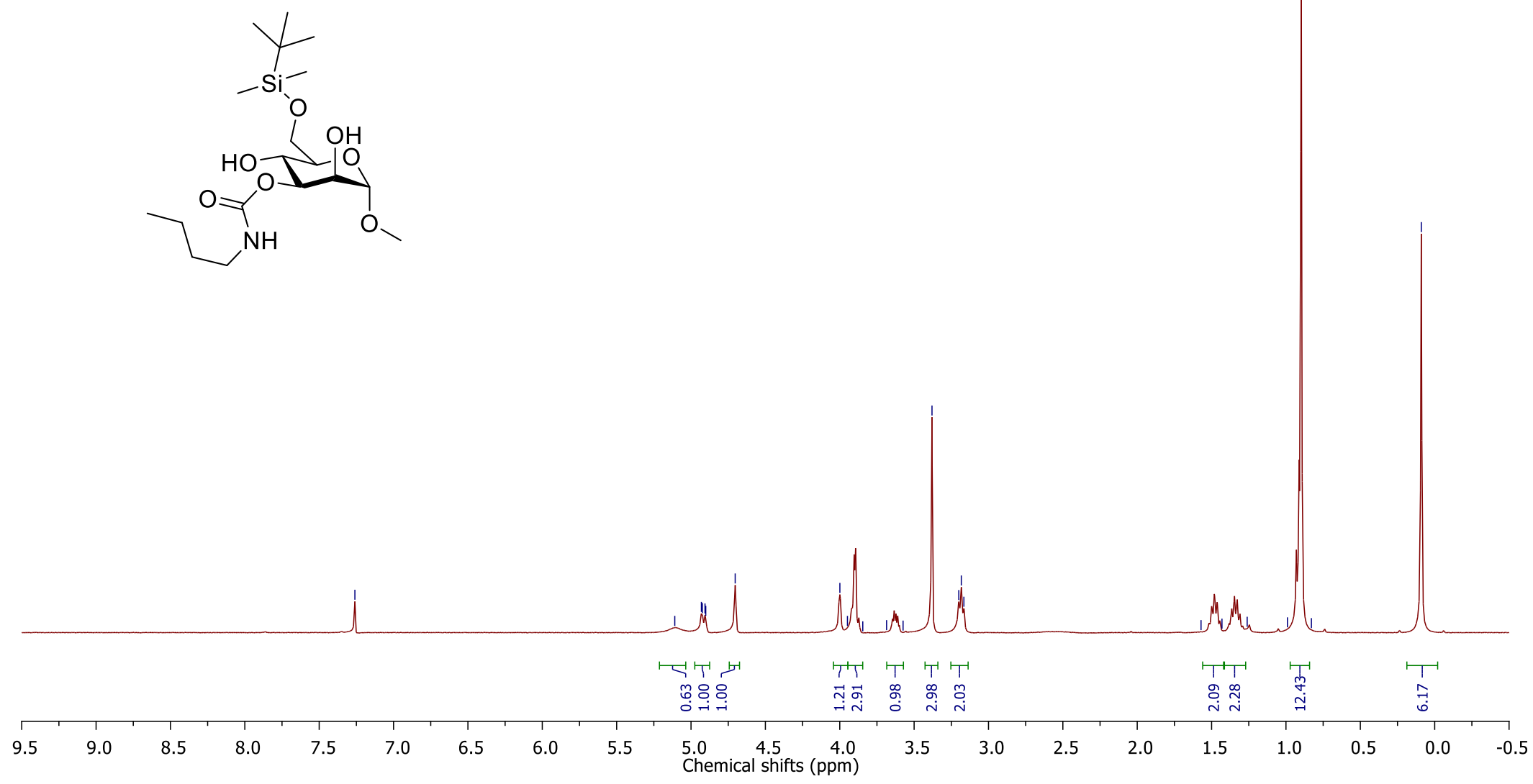
b. ${ }^{13} \mathrm{C}$ NMR spectrum $(101 \mathrm{~Hz})$ of methyl 3-O-butylcarbamoyl-6-O-(tert-butyldimethyl)silyl- $\alpha$-D-mannopyranoside (3c)

$3 \mathbf{c}\left(\mathrm{CDCl}_{3}\right)$

$\stackrel{\circ}{\stackrel{0}{1}}$

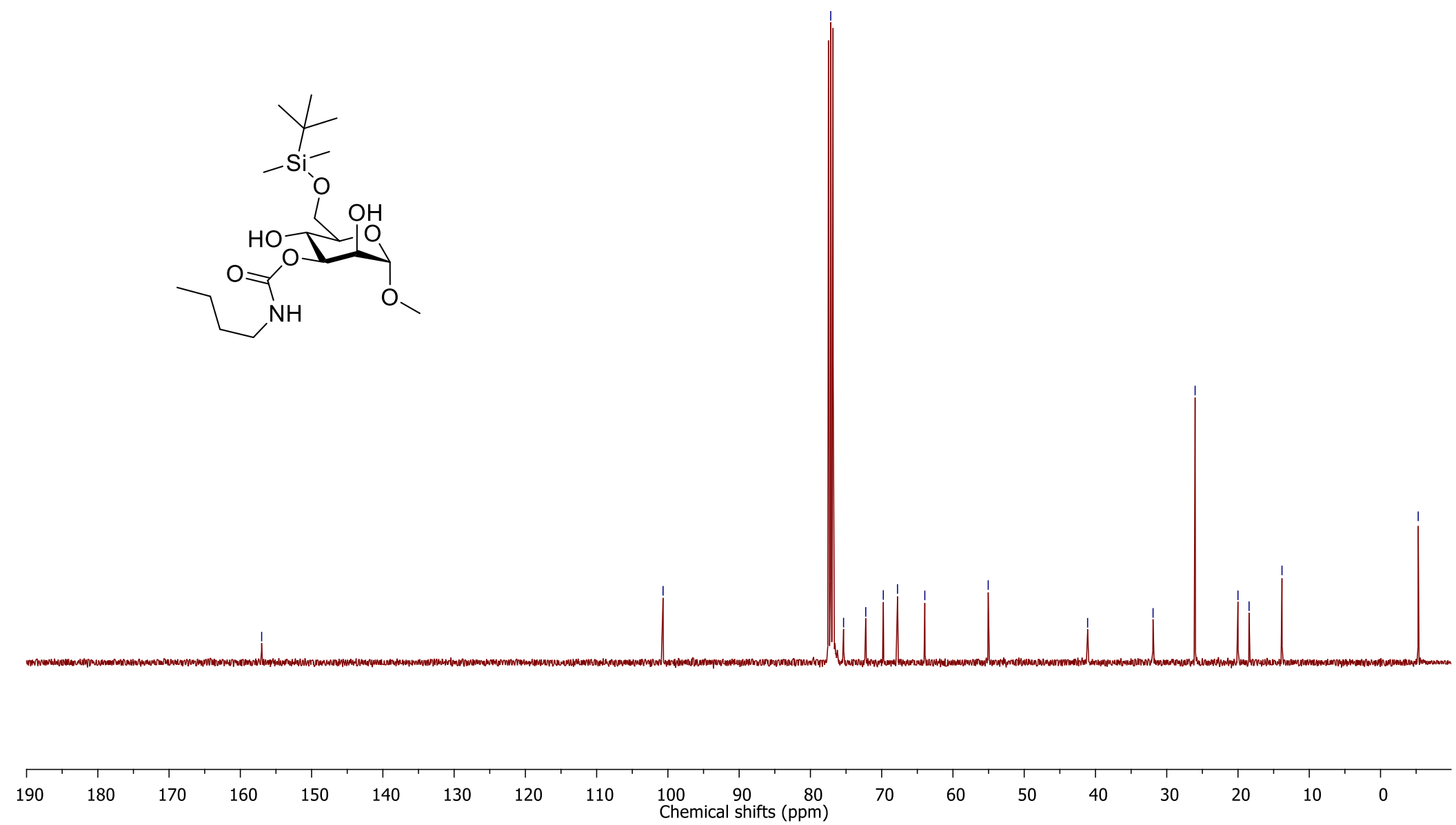

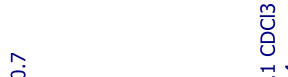

| 
c. COSY NMR spectrum $(400 \mathrm{~Hz})$ of methyl 3-O-butylcarbamoyl-6- $O$-(tert-butyldimethyl)silyl- $\alpha$-D-mannopyranoside (3c)

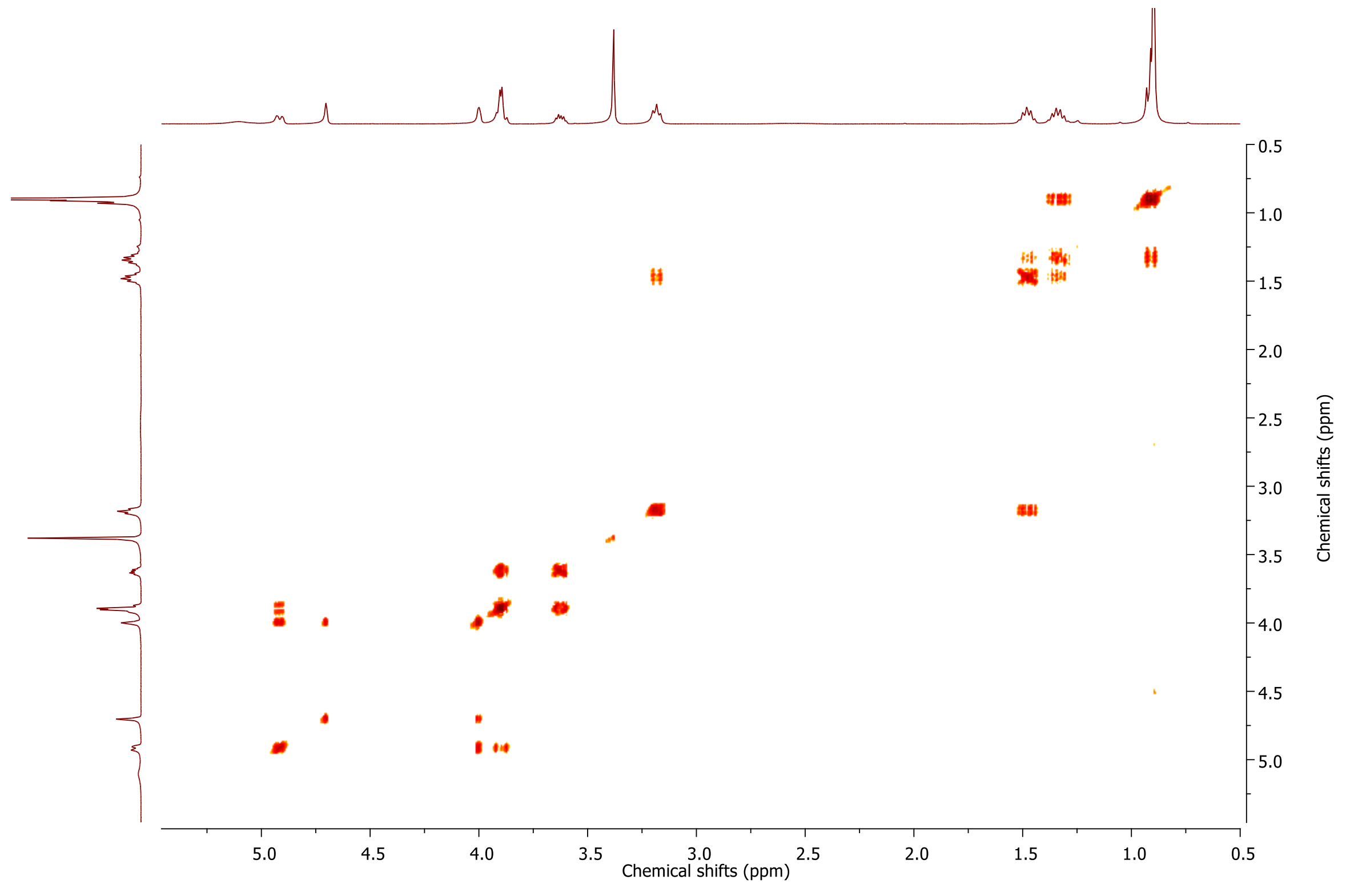


20. ${ }^{1} \mathrm{H},{ }^{13} \mathrm{C}$, and COSY NMR Spectra of compound $3 \mathrm{~d}$

a. ${ }^{1} \mathrm{H}$ NMR spectrum $(400 \mathrm{~Hz}$ ) of methyl 3-O-octylcarbamoyl-6-O-(tert-butyldimethyl)silyl- $\alpha$-D-mannopyranoside (3d) 3d $\left(\mathrm{CDCl}_{3}\right)$
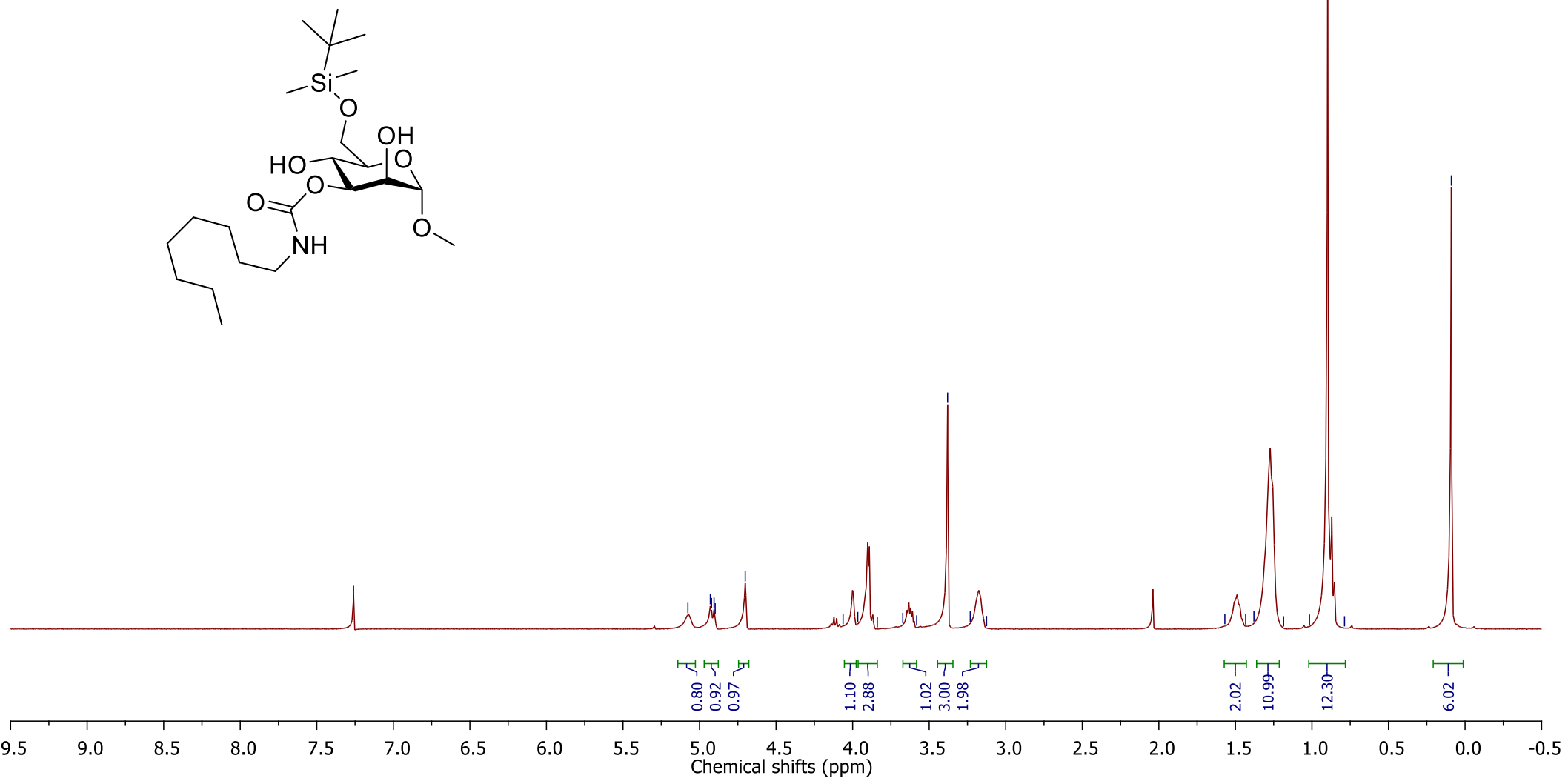
b. ${ }^{13} \mathrm{C}$ NMR spectrum $(101 \mathrm{~Hz})$ of methyl 3-O-octylcarbamoyl-6- $O$-(tert-butyldimethyl)silyl- $\alpha$-D-mannopyranoside (3d)

3d $\left(\mathrm{CDCl}_{3}\right)$

$\stackrel{\circ}{\stackrel{0}{1}}$

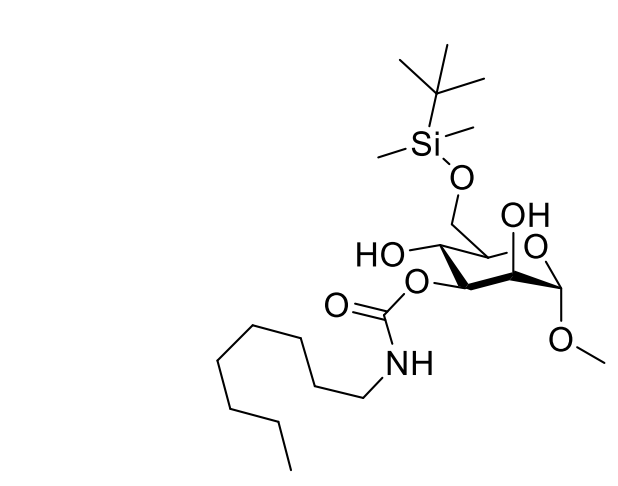

$\stackrel{m}{\circ}$

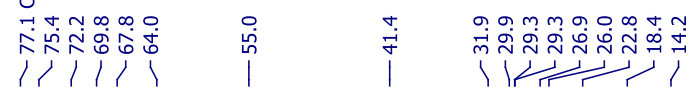

$90 \quad 80$
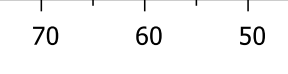

$40 \quad 30$

20

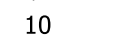


c. COSY NMR spectrum $(400 \mathrm{~Hz})$ of methyl 3-O-octylcarbamoyl-6- $O$-(tert-butyldimethyl)silyl- $\alpha$-D-mannopyranoside (3d)

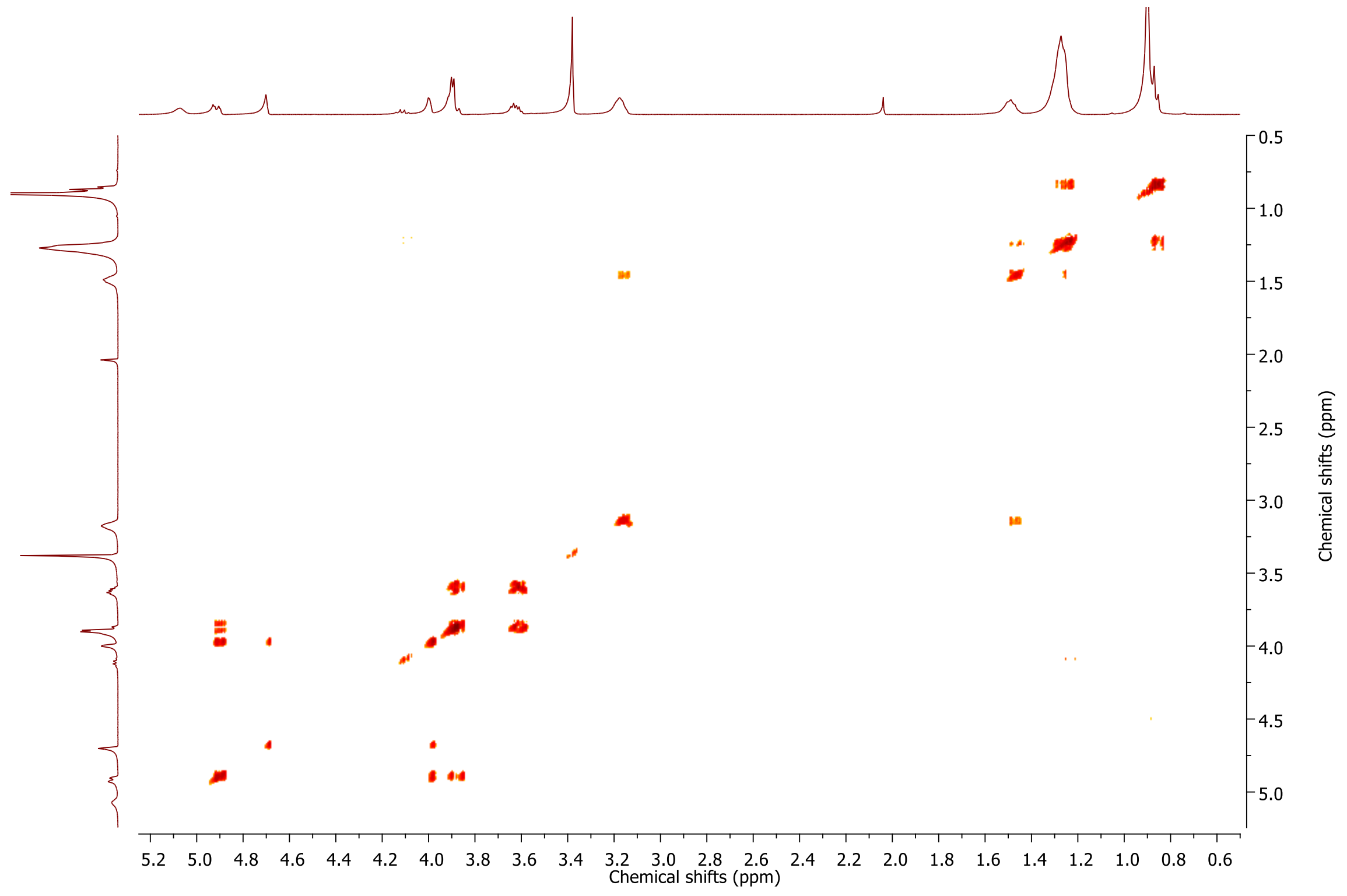


21. ${ }^{1} \mathrm{H},{ }^{13} \mathrm{C}$, and COSY NMR Spectra of compound $3 \boldsymbol{e}$

a. ${ }^{1} \mathrm{H}$ NMR spectrum $(400 \mathrm{~Hz})$ of methyl 3-O-cyclohexylcarbamoyl-6-O-(tert-butyldimethyl)silyl- $\alpha$-D-mannopyranoside (3e) 3e $\left(\mathrm{CDCl}_{3}\right)$
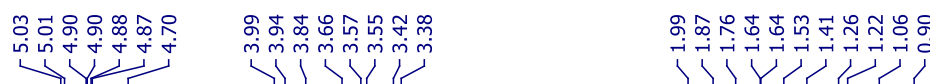

i
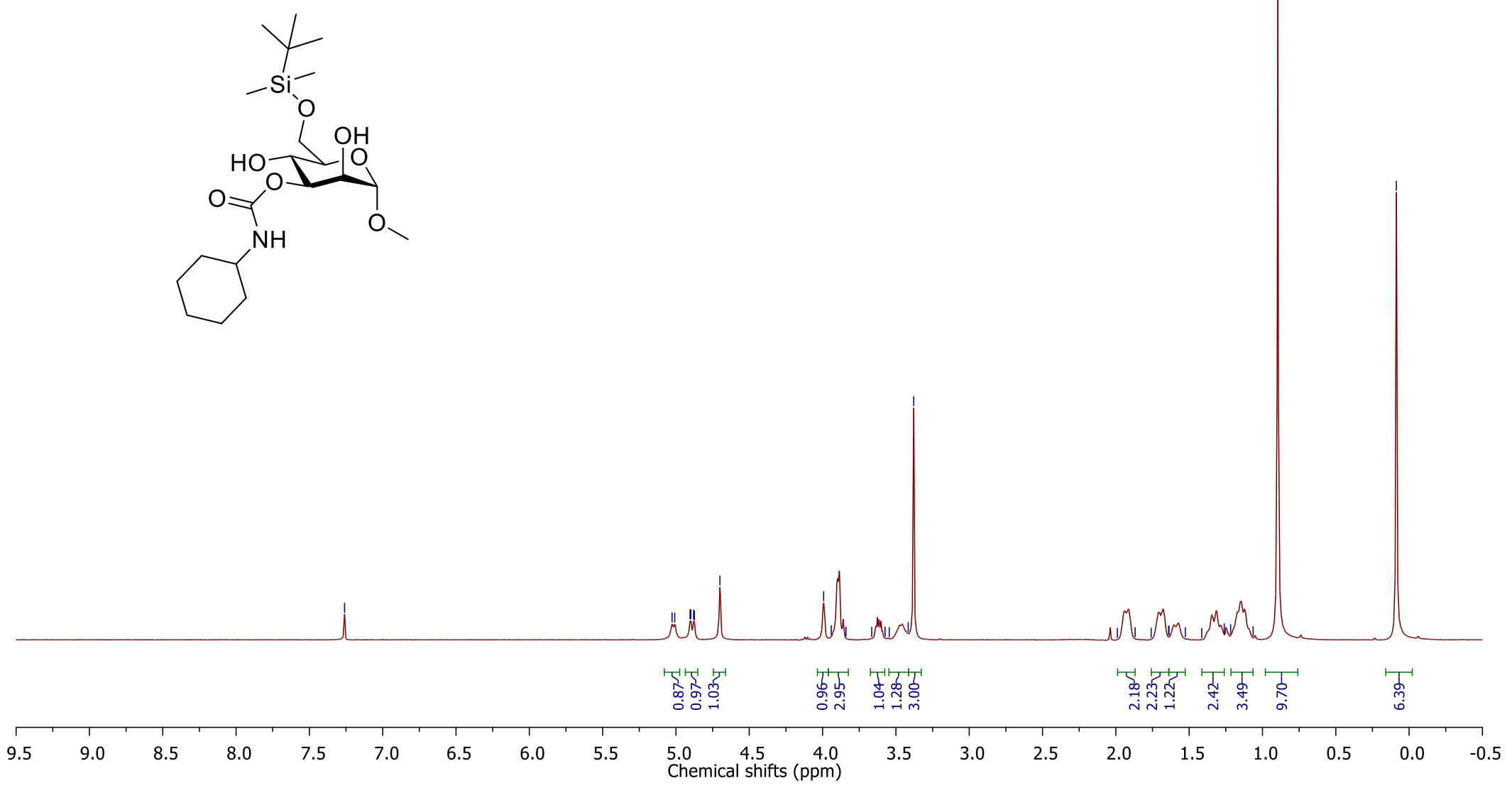
b. ${ }^{13} \mathrm{C}$ NMR spectrum $(101 \mathrm{~Hz})$ of methyl 3-O-cyclohexylcarbamoyl-6- $O$-(tert-butyldimethyl)silyl- $\alpha$-D-mannopyranoside (3e)

$3 \mathbf{e}\left(\mathrm{CDCl}_{3}\right)$

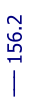

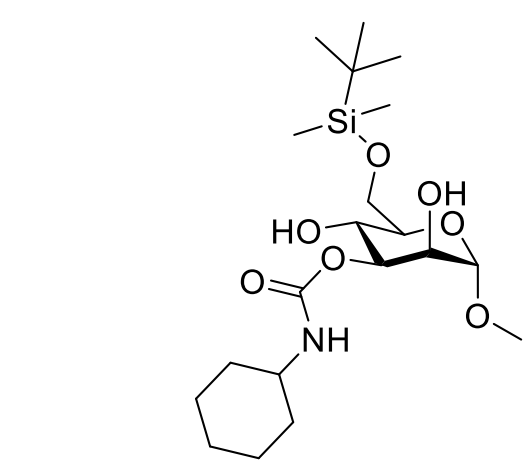

$\stackrel{0}{0.0}$

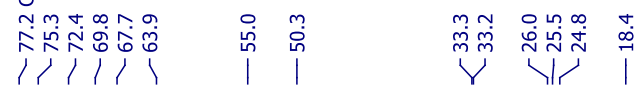
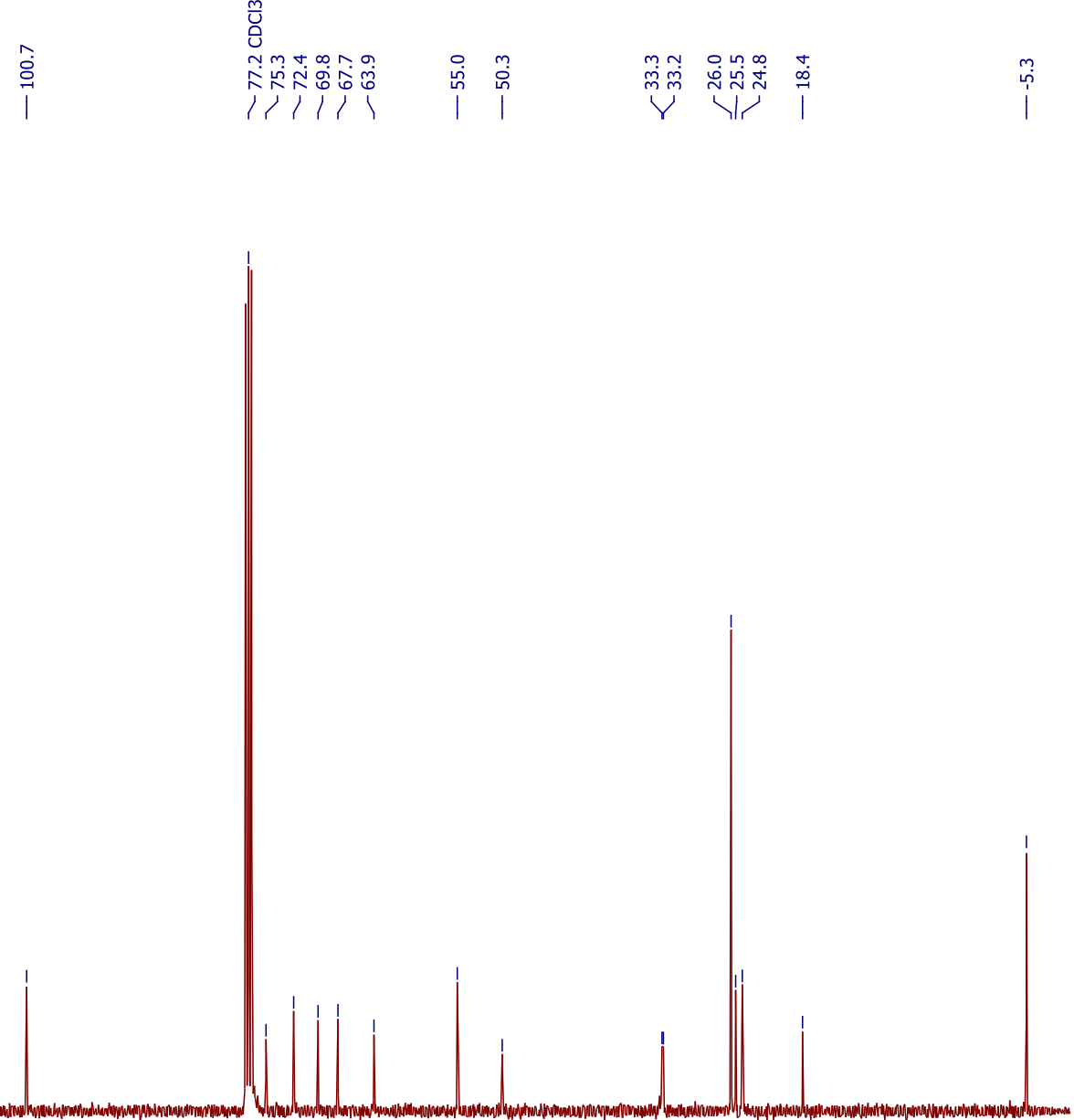

190 
c. COSY NMR spectrum $(400 \mathrm{~Hz})$ of methyl 3-O-cyclohexylcarbamoyl-6- $O$-(tert-butyldimethyl)silyl- $\alpha$-D-mannopyranoside (3e)

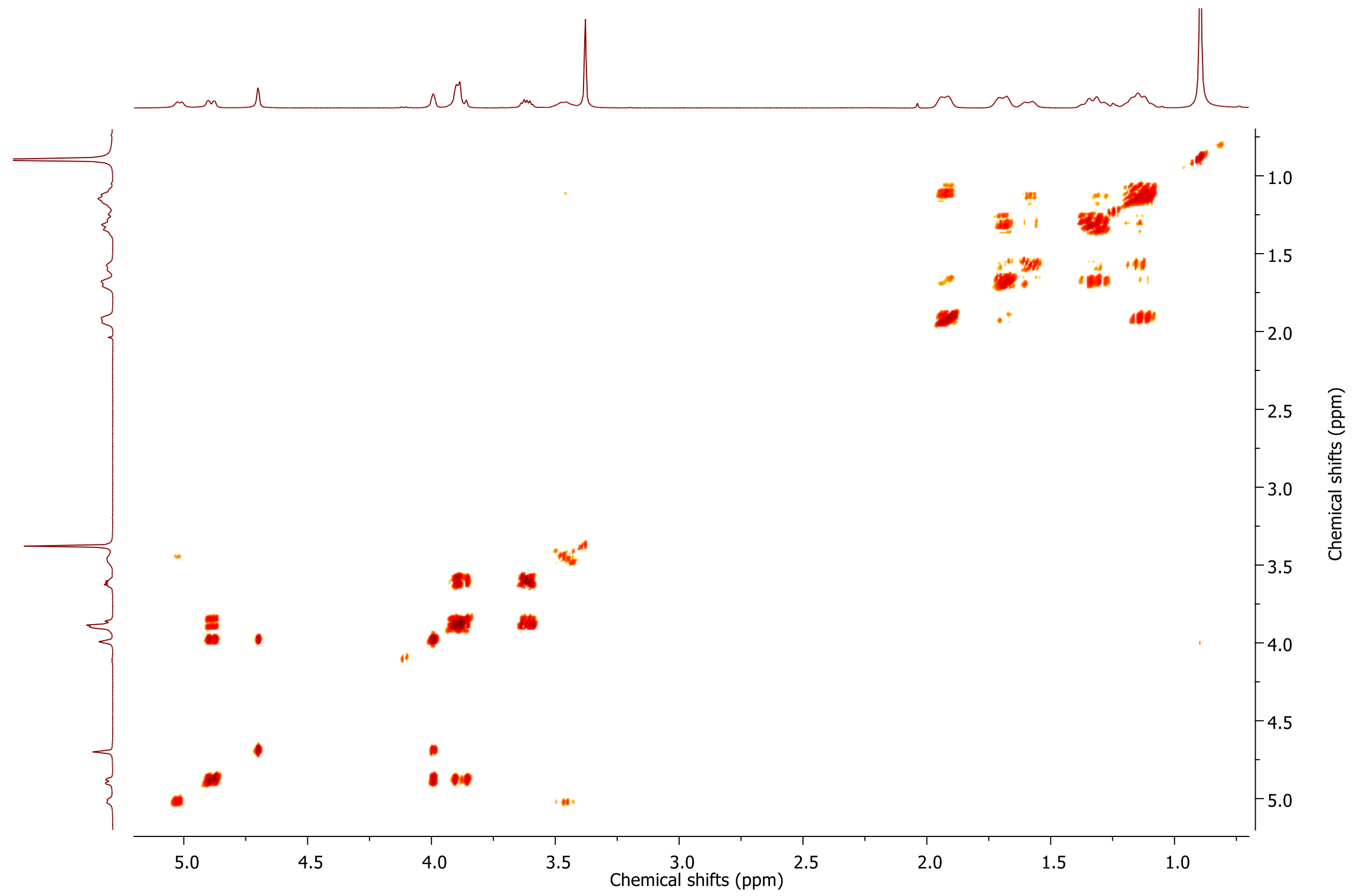


22. ${ }^{1} \mathrm{H},{ }^{13} \mathrm{C}$, and COSY NMR Spectra of compound $7 \boldsymbol{a}$

a. ${ }^{1} \mathrm{H}$ NMR spectrum $(400 \mathrm{~Hz})$ of methyl 3-O-benzylcarbamoyl-6-O-(tert-butyldimethyl)silyl- $\beta$-D-galactopyranoside (7a)

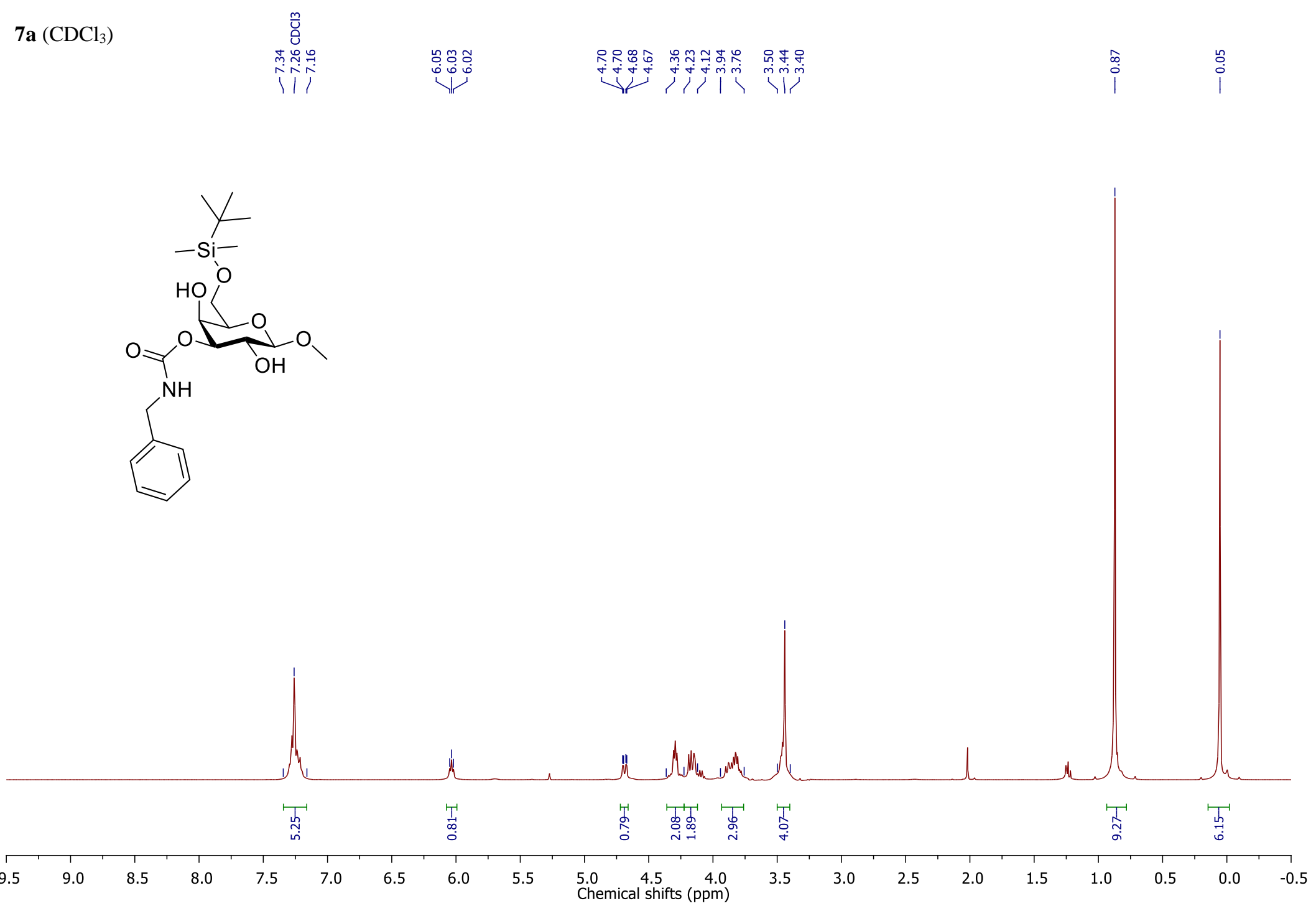


b. ${ }^{13} \mathrm{C}$ NMR spectrum $(101 \mathrm{~Hz})$ of methyl 3-O-benzylcarbamoyl-6- $O$-(tert-butyldimethyl)silyl- $\beta$-D-galactopyranoside (7a)

$7 \mathbf{a}\left(\mathrm{CDCl}_{3}\right)$

i.

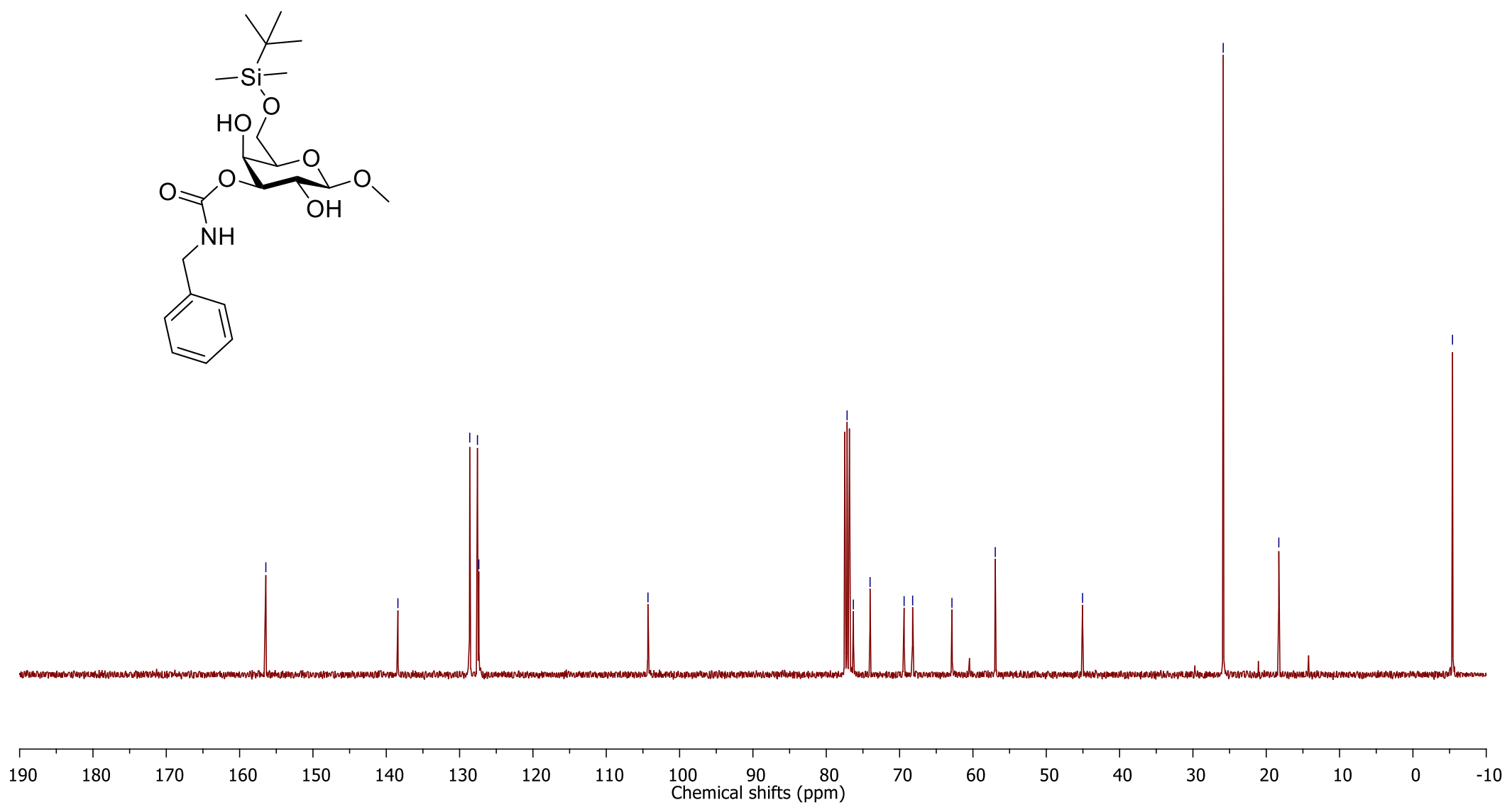

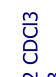

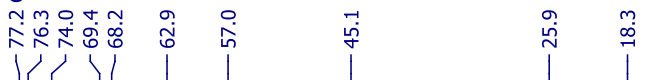

$\stackrel{i}{\dot{0}}$ 
c. COSY NMR spectrum (400 Hz) of methyl 3-O-benzylcarbamoyl-6-O-(tert-butyldimethyl)silyl- $\beta$-D-galactopyranoside (7a)

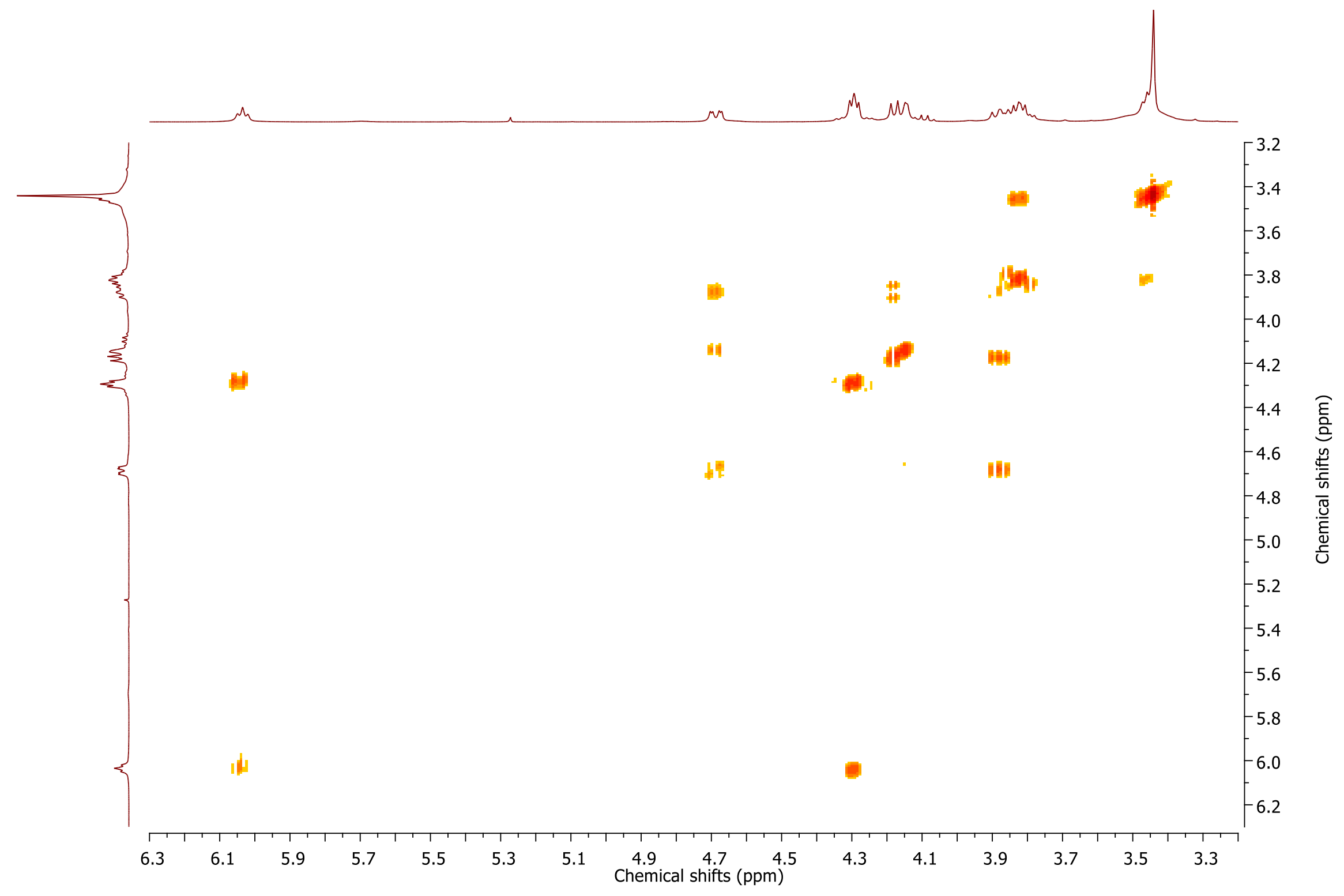


23. ${ }^{1} \mathrm{H},{ }^{13} \mathrm{C}$, and COSY NMR Spectra of compound $\mathbf{7 b}$

a. ${ }^{1} \mathrm{H}$ NMR spectrum $(400 \mathrm{~Hz})$ of methyl 3-O-benzylcarbamoyl-6-O-(tert-butyldimethyl)silyl- $\alpha$-D-galactopyranoside (7b) $\mathbf{7 b}\left(\mathrm{CDCl}_{3}\right)$
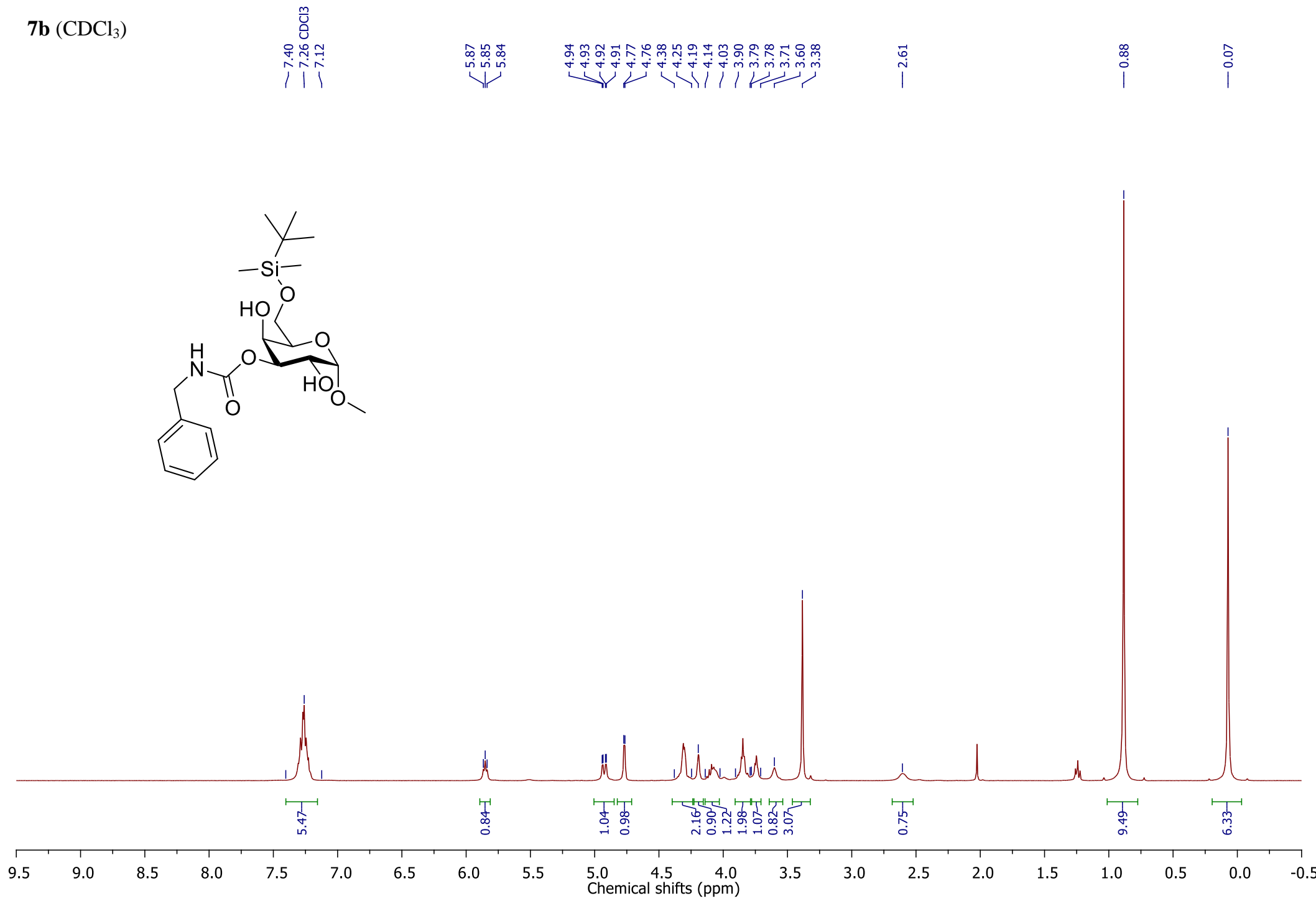
b. ${ }^{13} \mathrm{C}$ NMR spectrum $(101 \mathrm{~Hz})$ of methyl 3-O-benzylcarbamoyl-6- $O$-(tert-butyldimethyl)silyl- $\alpha$-D-galactopyranoside (7b)

7b $\left(\mathrm{CDCl}_{3}\right)$

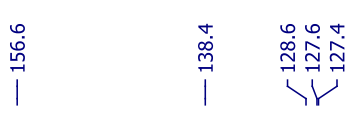

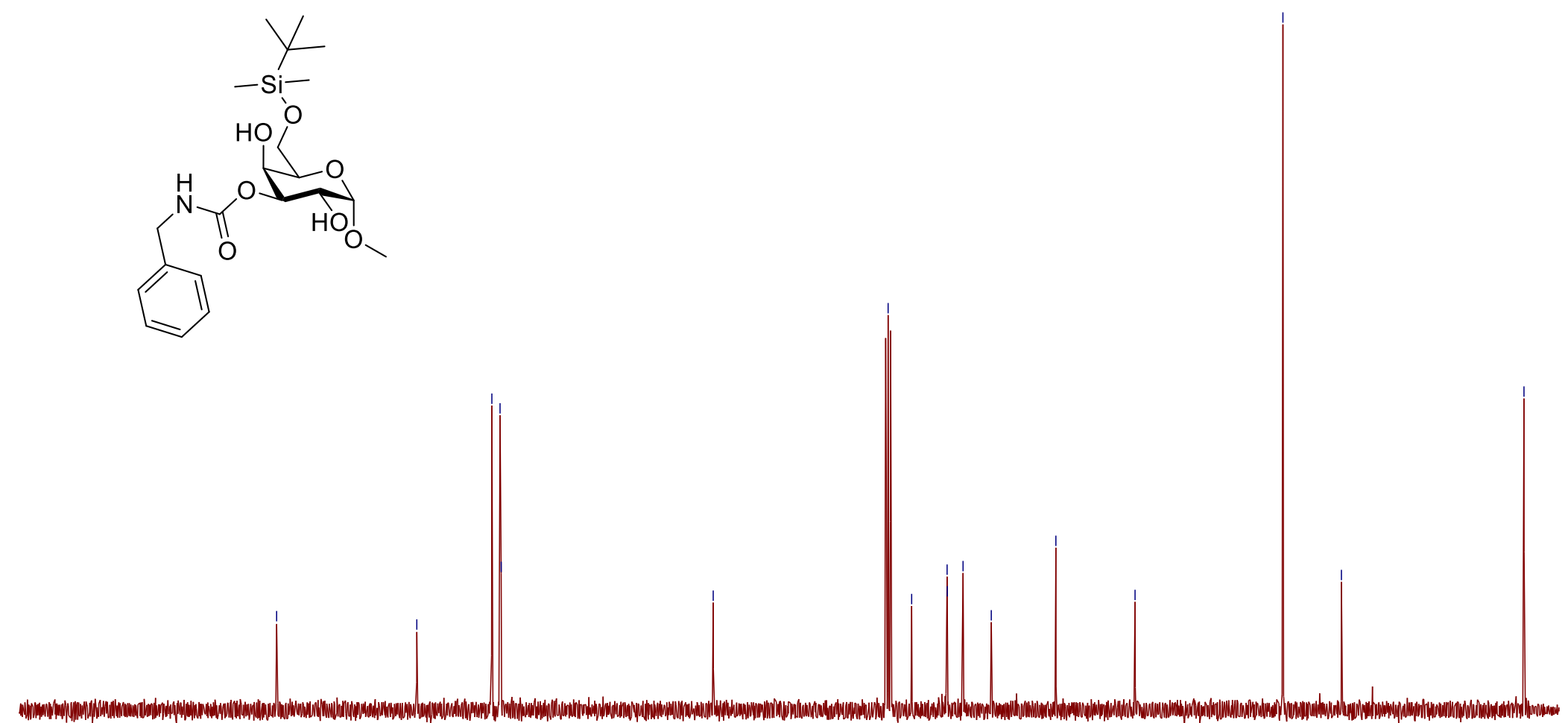

రิ)

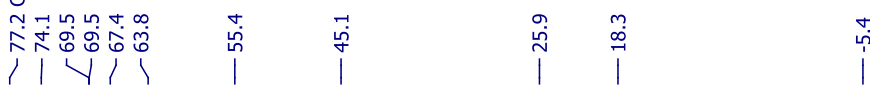


c. COSY NMR spectrum $(400 \mathrm{~Hz}$ ) of methyl 3-O-benzylcarbamoyl-6-O-(tert-butyldimethyl)silyl- $\alpha$-D-galactopyranoside (7b)

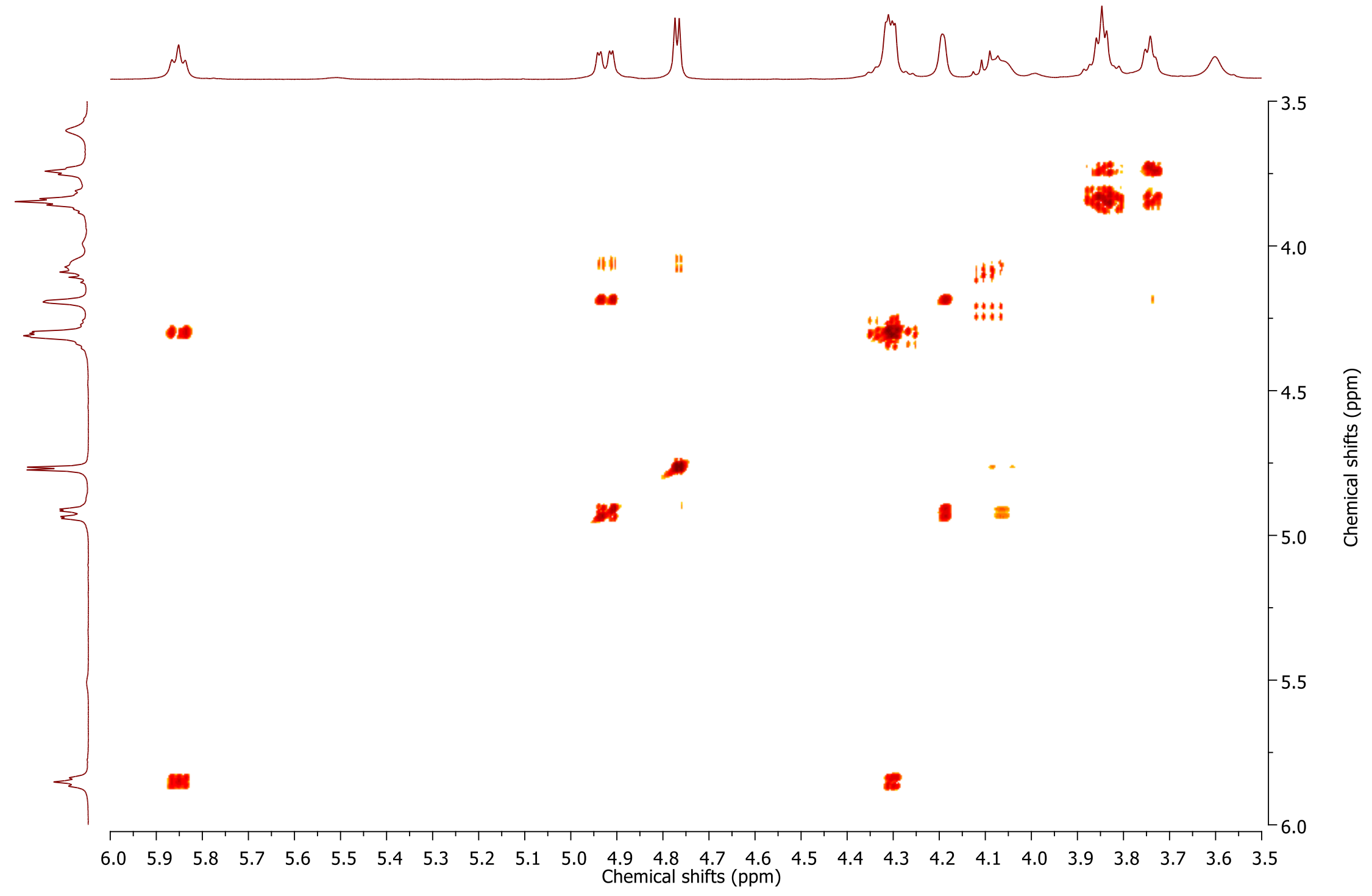


24. ${ }^{1} \mathrm{H},{ }^{13} \mathrm{C}$, and COSY NMR Spectra of compound $9 a$

a. ${ }^{1} \mathrm{H}$ NMR spectrum $(400 \mathrm{~Hz})$ of 3-O-benzylcarbamoyl-6- $O$-(tert-butyldimethyl)silyl- $\alpha$-D-mannopyranoside (9a)

9a $\left(\mathrm{CDCl}_{3}\right)$

岩

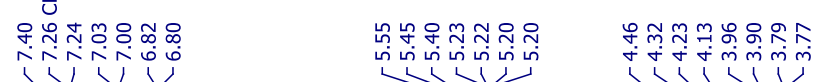

i
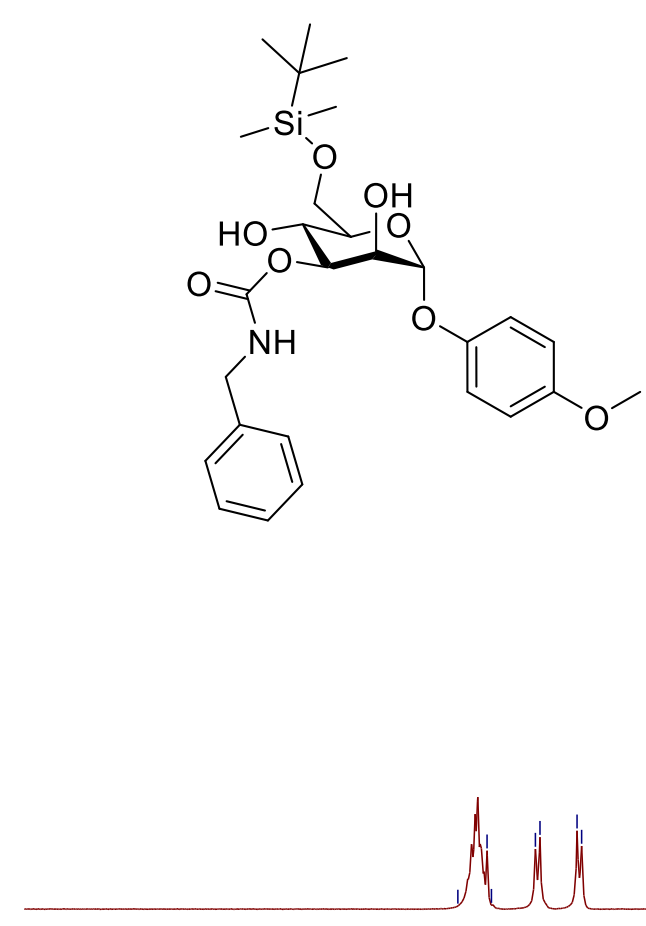

oln
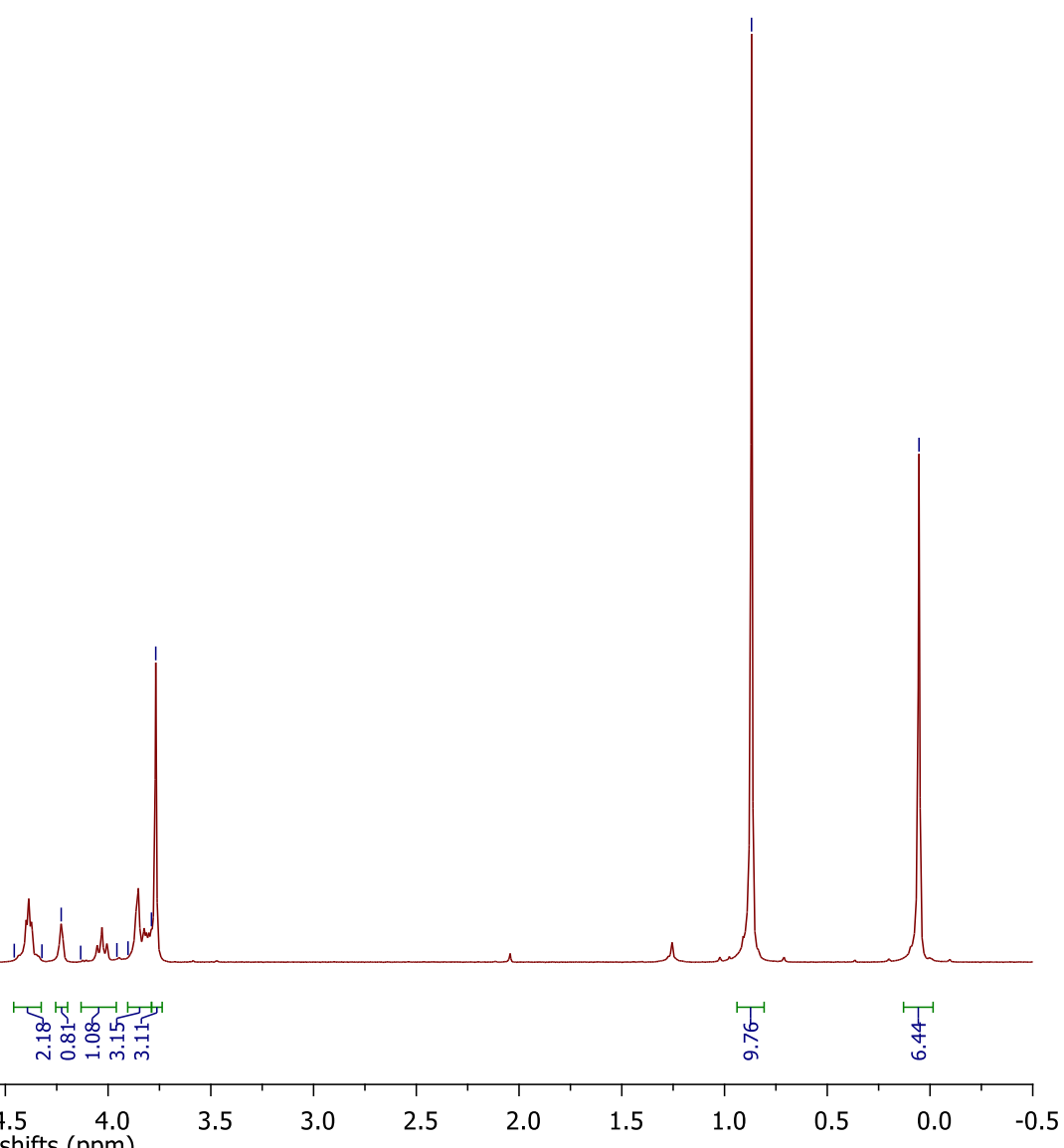

9.5

9.0

$8.5 \quad 8.0$

声 祭尔

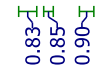

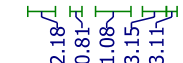

3.5

3.0

2.5

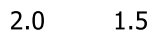


b. ${ }^{13} \mathrm{C}$ NMR spectrum $(101 \mathrm{~Hz})$ of 4-methoxyphenyl 3-O-benzylcarbamoyl-6-O-(tert-butyldimethyl)silyl- $\alpha$-D-mannopyranoside (9a)

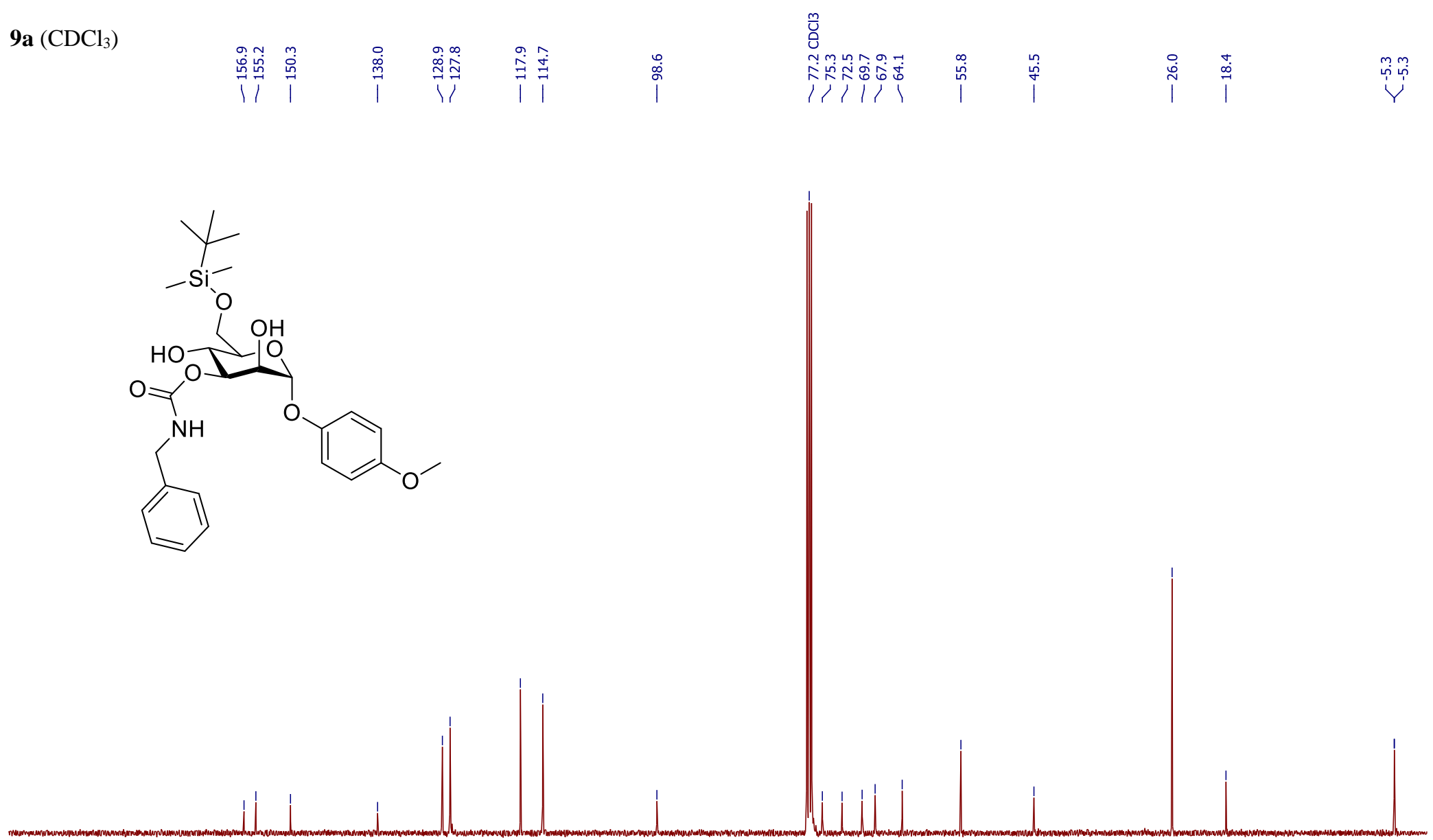

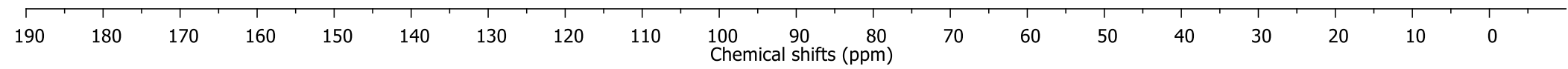


c. COSY NMR spectrum $(400 \mathrm{~Hz})$ of 4-methoxyphenyl 3-O-benzylcarbamoyl-6-O-(tert-butyldimethyl)silyl- $\alpha$-Dmannopyranoside (9a)

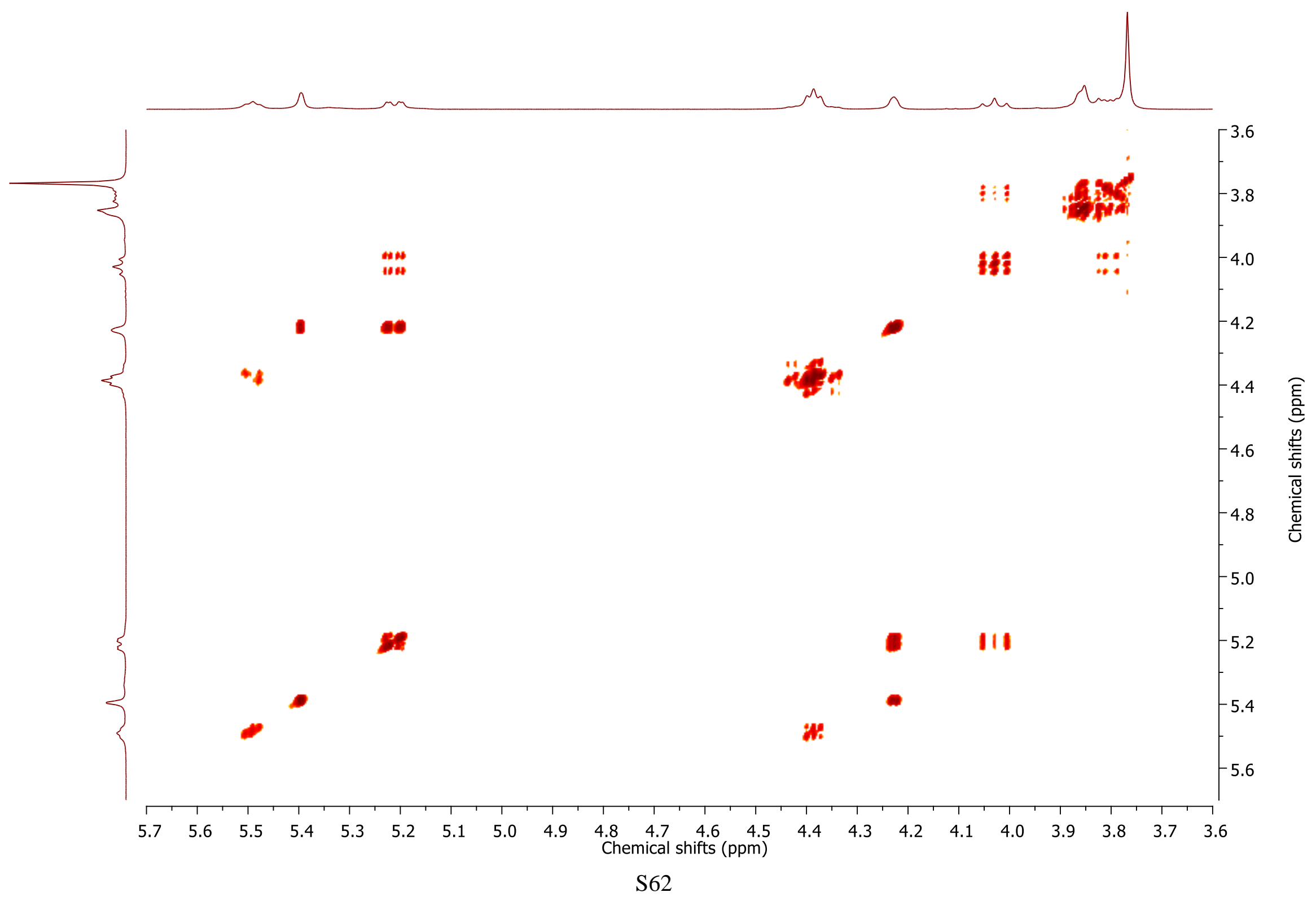


25. ${ }^{1} \mathrm{H},{ }^{13} \mathrm{C}$, and COSY NMR Spectra of compound $\mathbf{9 b}$

a. $\quad{ }^{1} \mathrm{H}$ NMR spectrum $(400 \mathrm{~Hz})$ of 3-O-benzylcarbamoyl-6- $O$-(tert-butyldimethyl)silyl- $\beta$-D-galactopyranoside (9b)

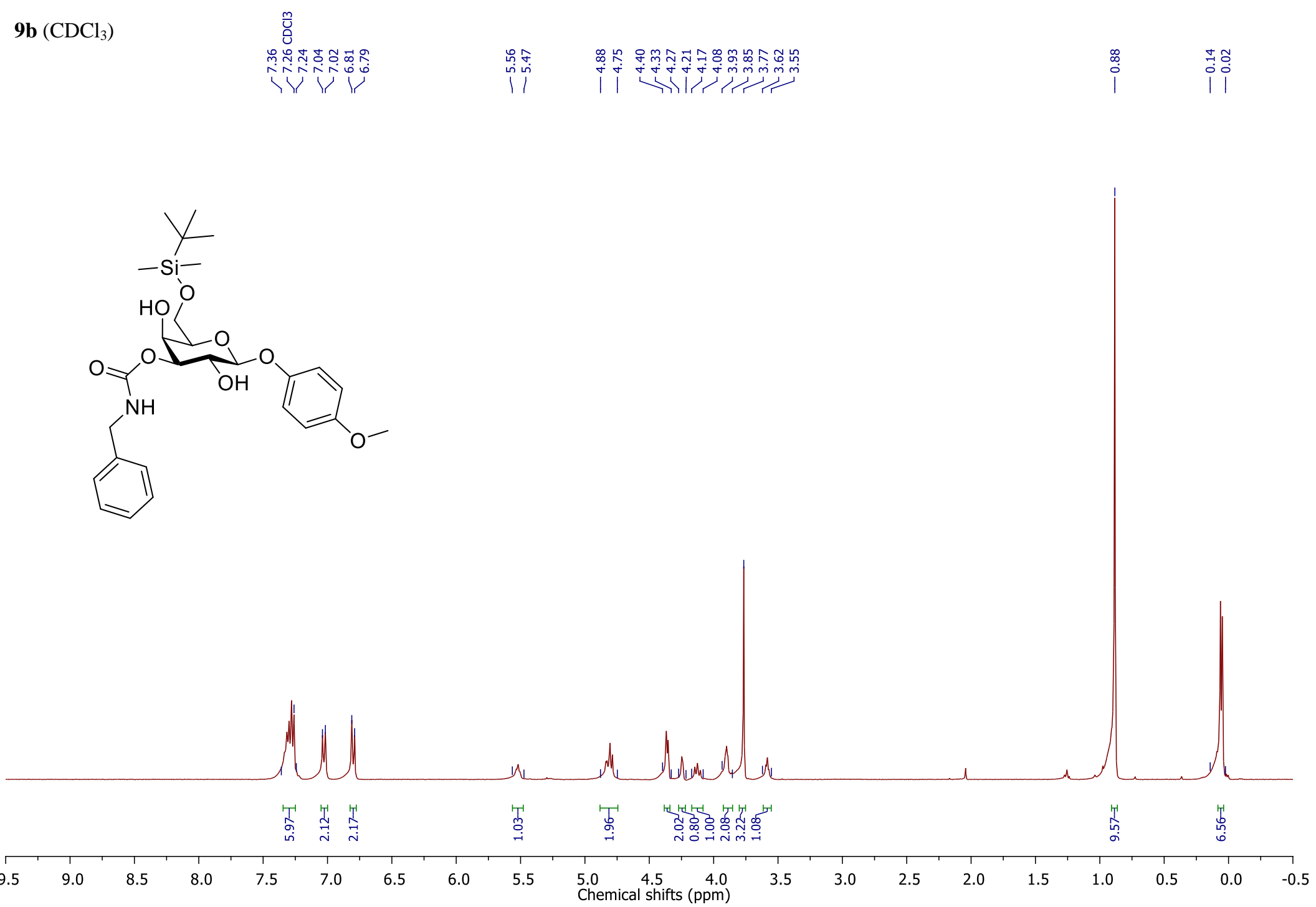


b. ${ }^{13} \mathrm{C}$ NMR spectrum $(101 \mathrm{~Hz})$ of 4-methoxyphenyl 3-O-benzylcarbamoyl-6- $O$-(tert-butyldimethyl)silyl- $\beta$-D-galactopyranoside (9b)

9b $\left(\mathrm{CDCl}_{3}\right)$

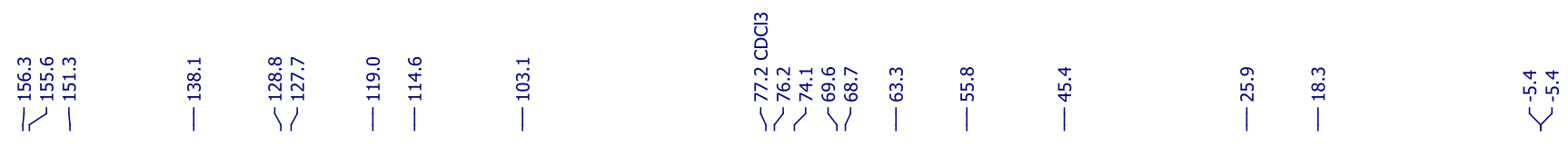
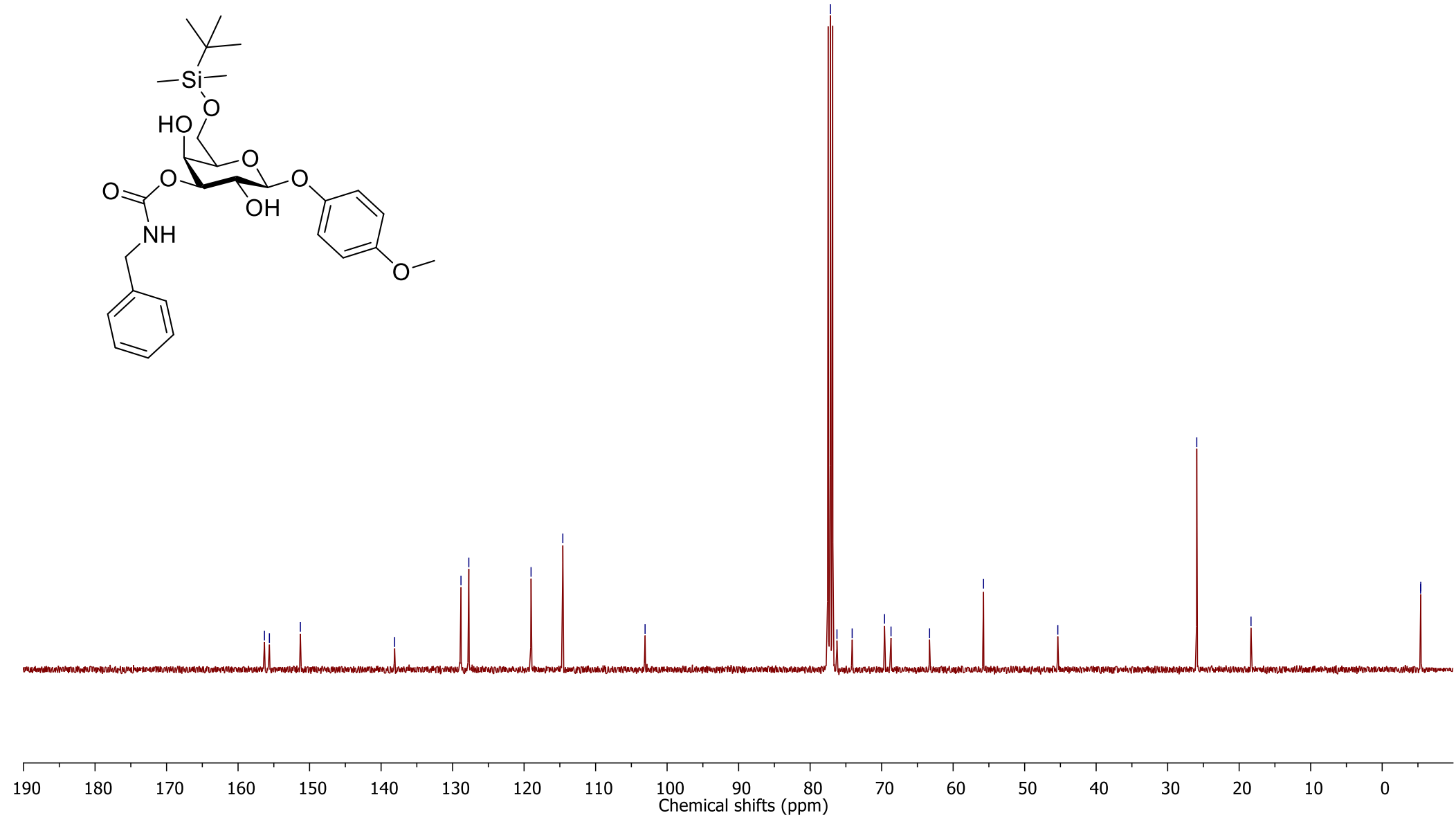
c. COSY NMR spectrum $(400 \mathrm{~Hz})$ of 4-methoxyphenyl 3-O-benzylcarbamoyl-6-O-(tert-butyldimethyl)silyl- $\beta$-Dgalactopyranoside (9b)

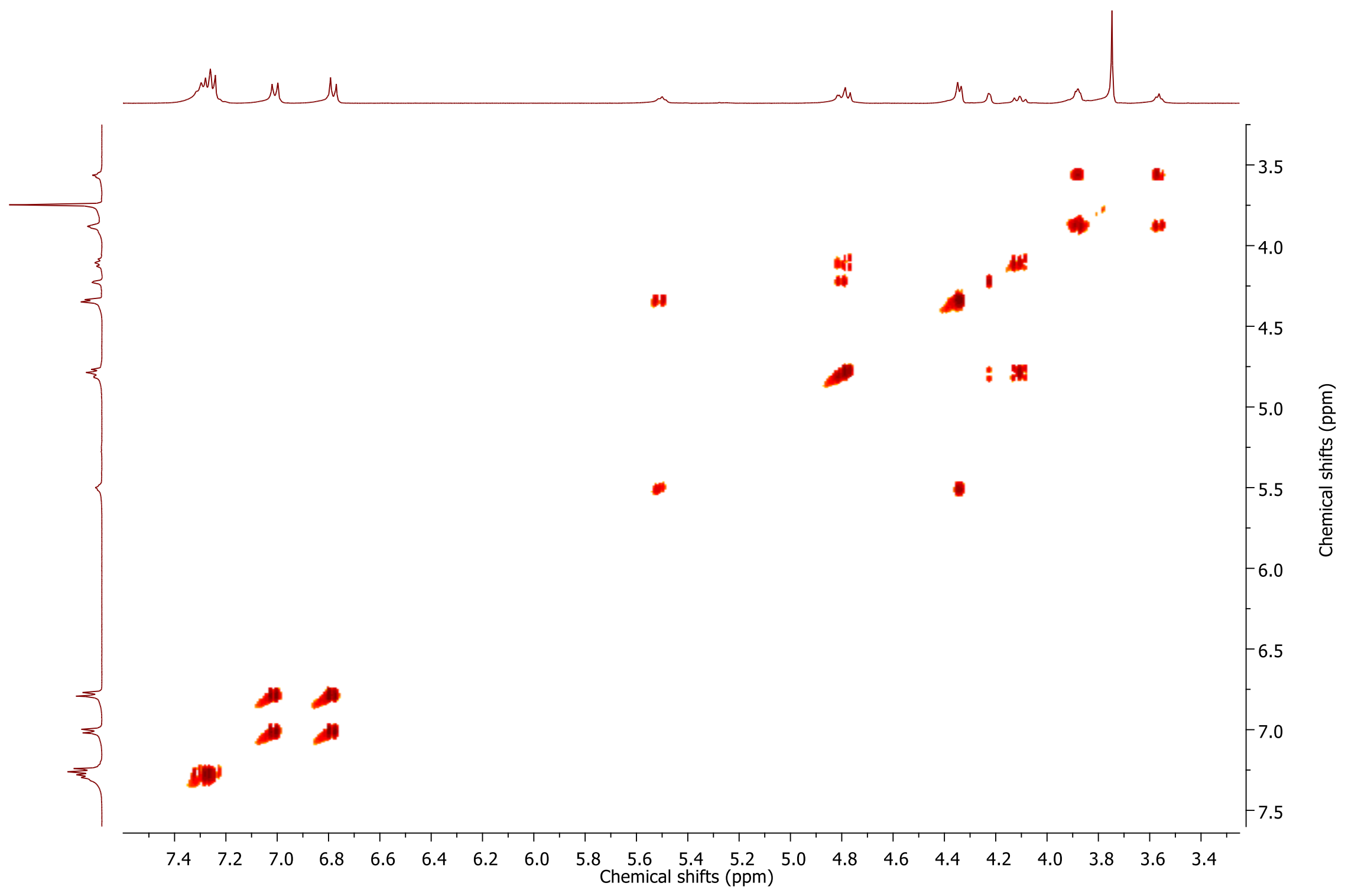


26. ${ }^{1} \mathrm{H},{ }^{13} \mathrm{C}$, and COSY NMR Spectra of compound $9 \mathrm{c}$

a. ${ }^{1} \mathrm{H}$ NMR spectrum $(400 \mathrm{~Hz})$ of 4-methoxyphenyl 3-O-benzylcarbamoyl- $\alpha$-L-arabinopyranoside (9c)
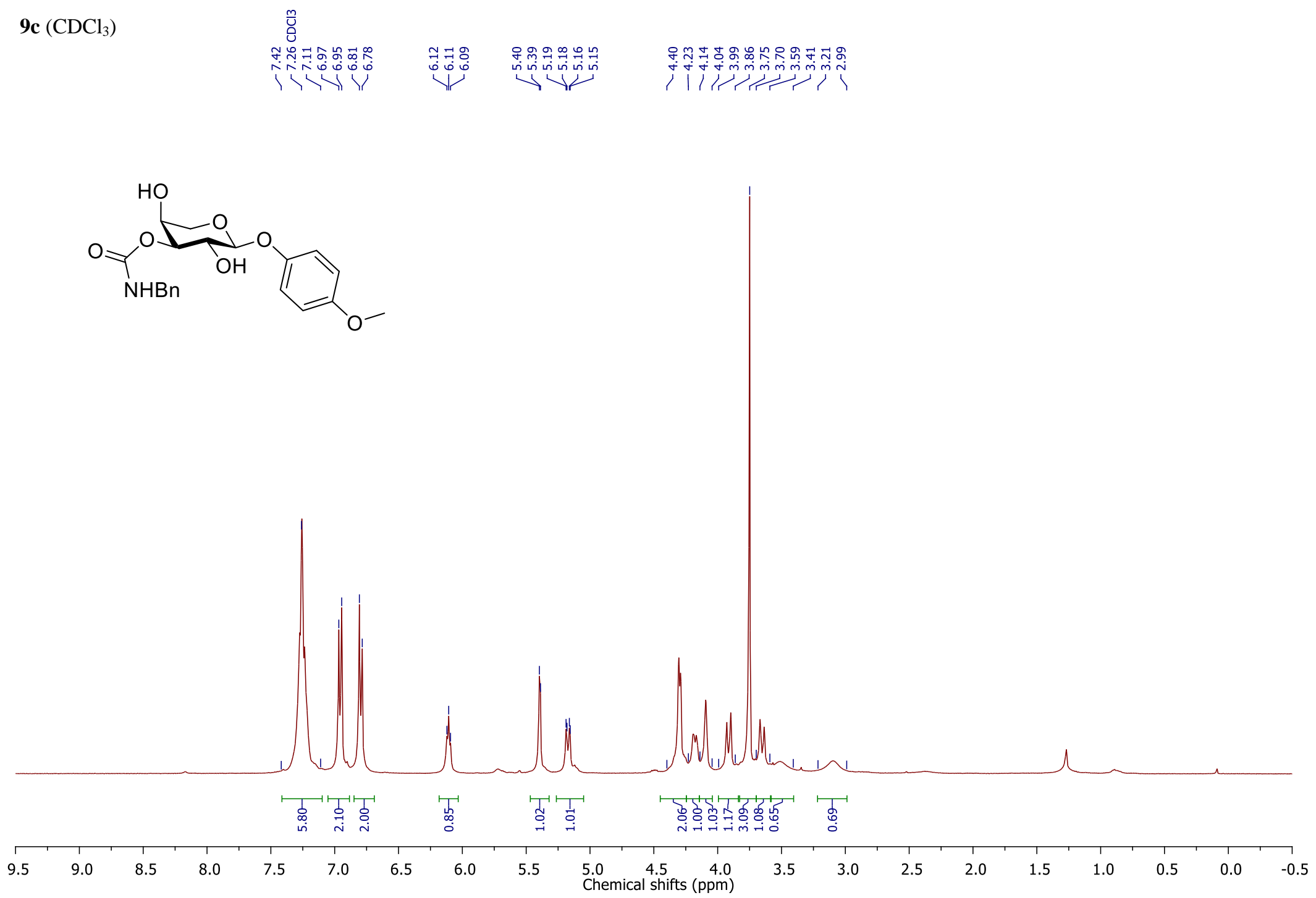
b. ${ }^{13} \mathrm{C}$ NMR spectrum $(101 \mathrm{~Hz})$ of 4-methoxyphenyl 3-O-benzylcarbamoyl- $\alpha$-L-arabinopyranoside $(\mathbf{9 c})$

$9 \mathbf{c}\left(\mathrm{CDCl}_{3}\right)$

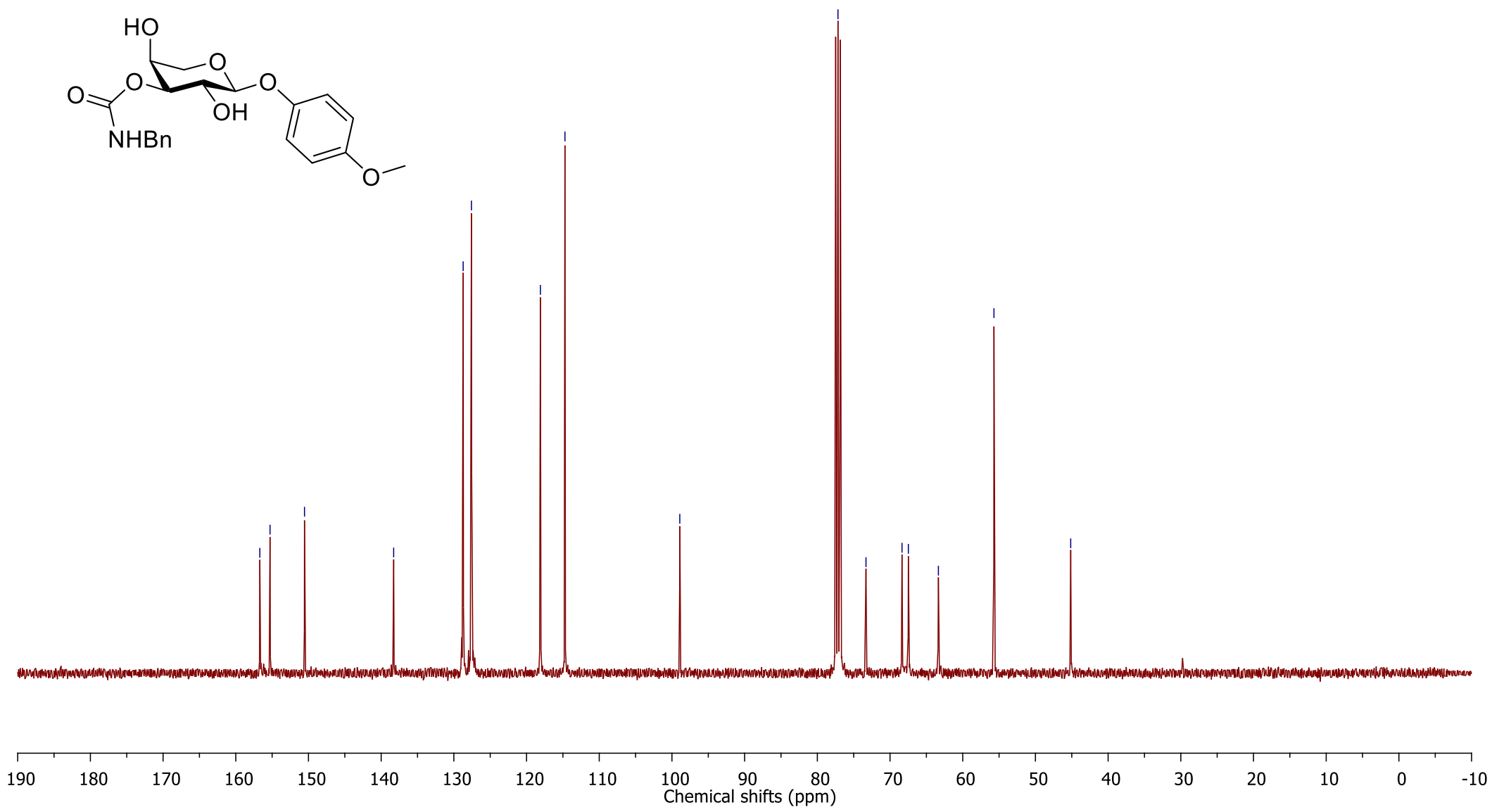


c. COSY NMR spectrum $(400 \mathrm{~Hz})$ of 4-methoxyphenyl 3-O-benzylcarbamoyl- $\alpha$-L-arabinopyranoside (9c)

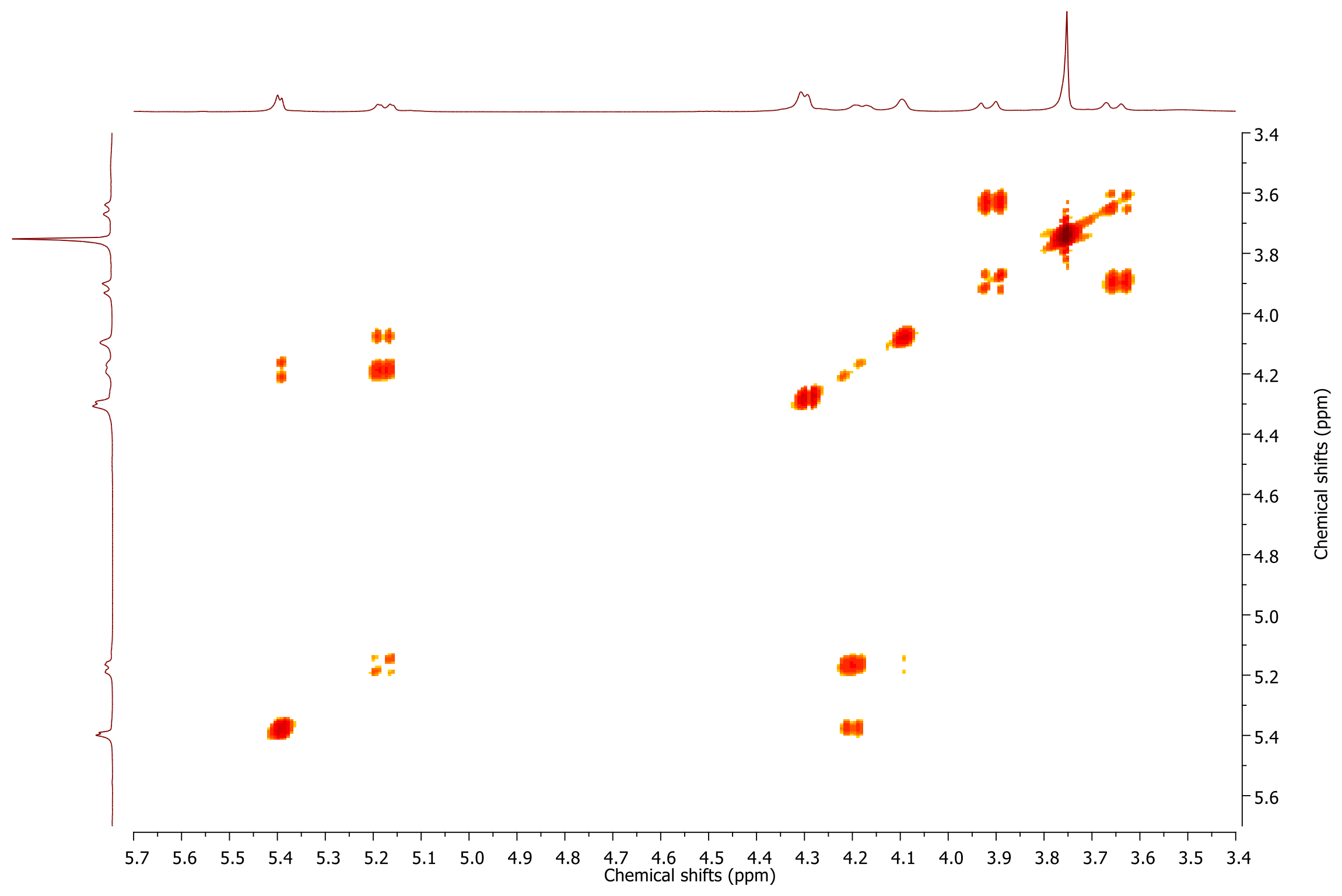


27. ${ }^{1} \mathrm{H},{ }^{13} \mathrm{C}$, and COSY NMR Spectra of compound $9 \boldsymbol{d}$

a. ${ }^{1} \mathrm{H}$ NMR spectrum $(400 \mathrm{~Hz})$ of 4-methoxyphenyl 3-O-benzylcarbamoyl- $\alpha$-L-lyxopyranoside $(\mathbf{9 d})$

9d $\left(\mathrm{CD}_{3} \mathrm{OD}\right)$
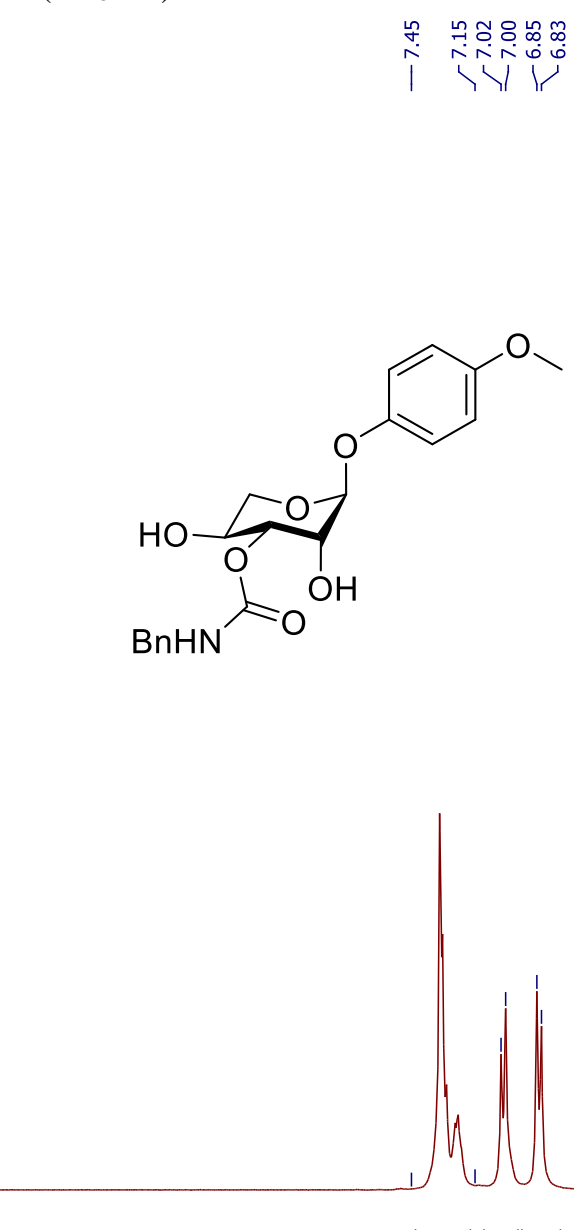

শูํำ

9.5

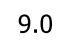

8.5
8.0

7.5

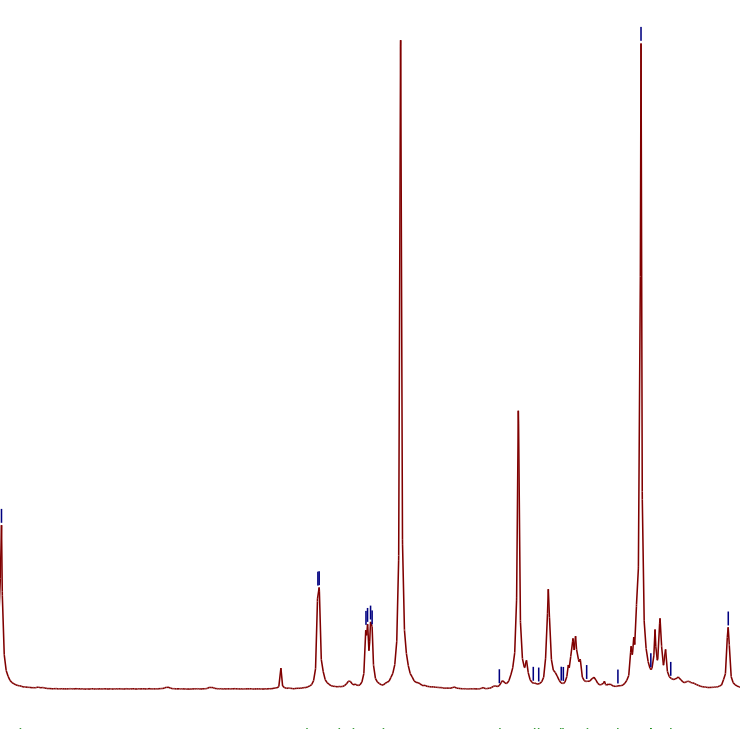

6.5

6.0

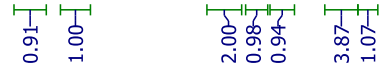

$\begin{array}{llr}5.0 & 4.5 & 4.0 \\ \text { Chemical shifts } & (\mathrm{ppm})\end{array}$ 
b. ${ }^{13} \mathrm{C}$ NMR spectrum $(101 \mathrm{~Hz})$ of 4-methoxyphenyl 3-O-benzylcarbamoyl- $\alpha$-L-lyxopyranoside (9d)

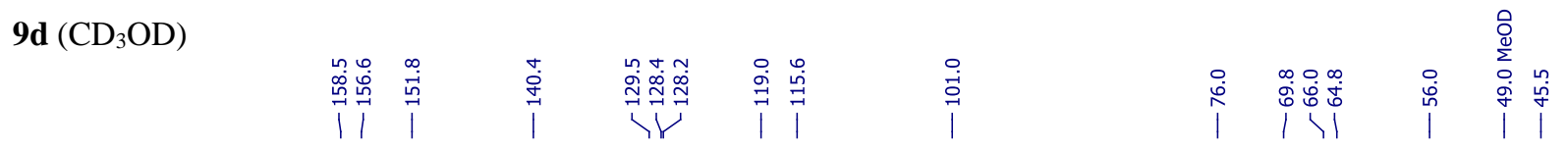
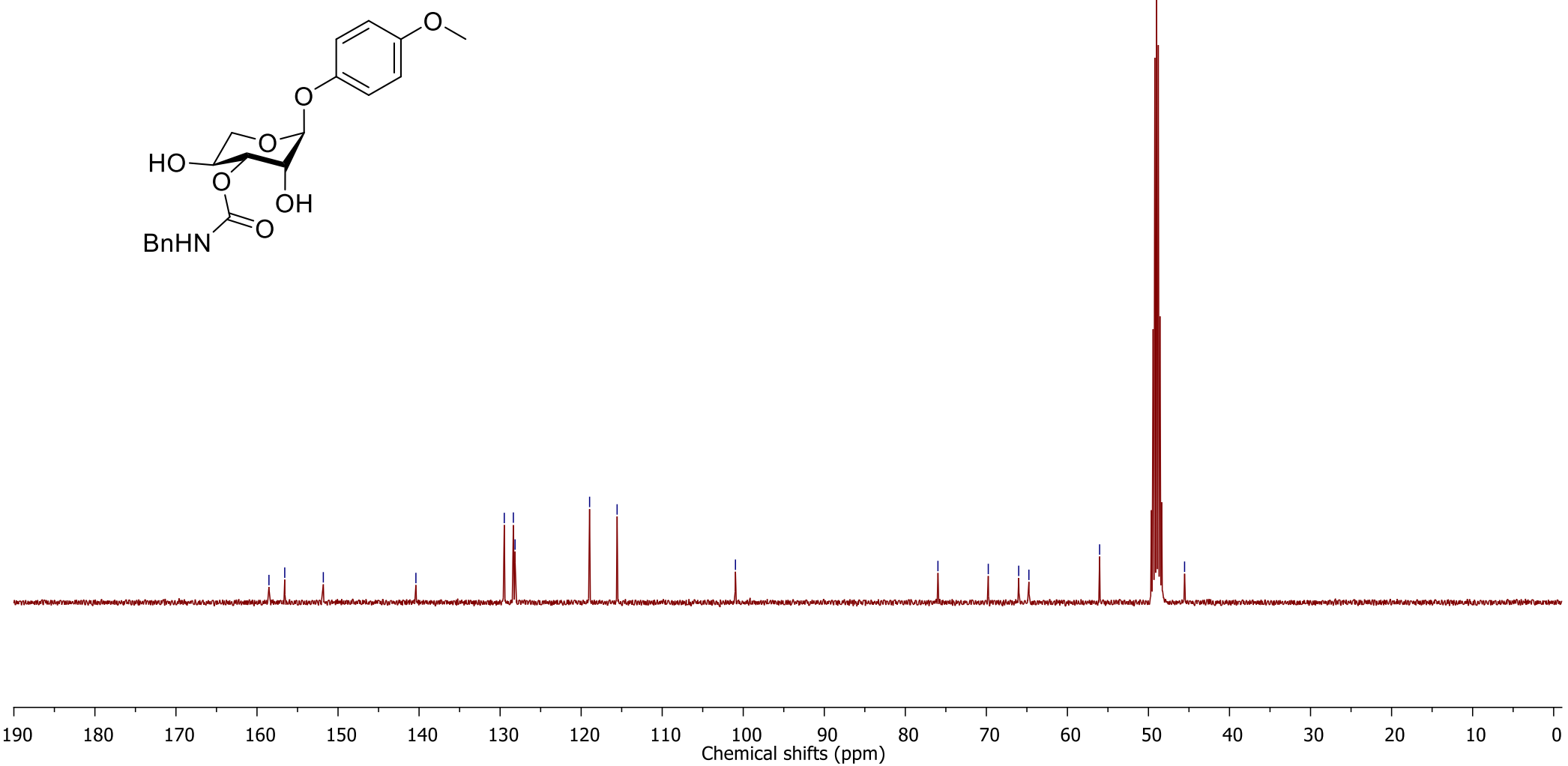
c. COSY NMR spectrum (400 Hz) of 4-methoxyphenyl 3-O-benzylcarbamoyl- $\alpha$-L-lyxopyranoside (9d)

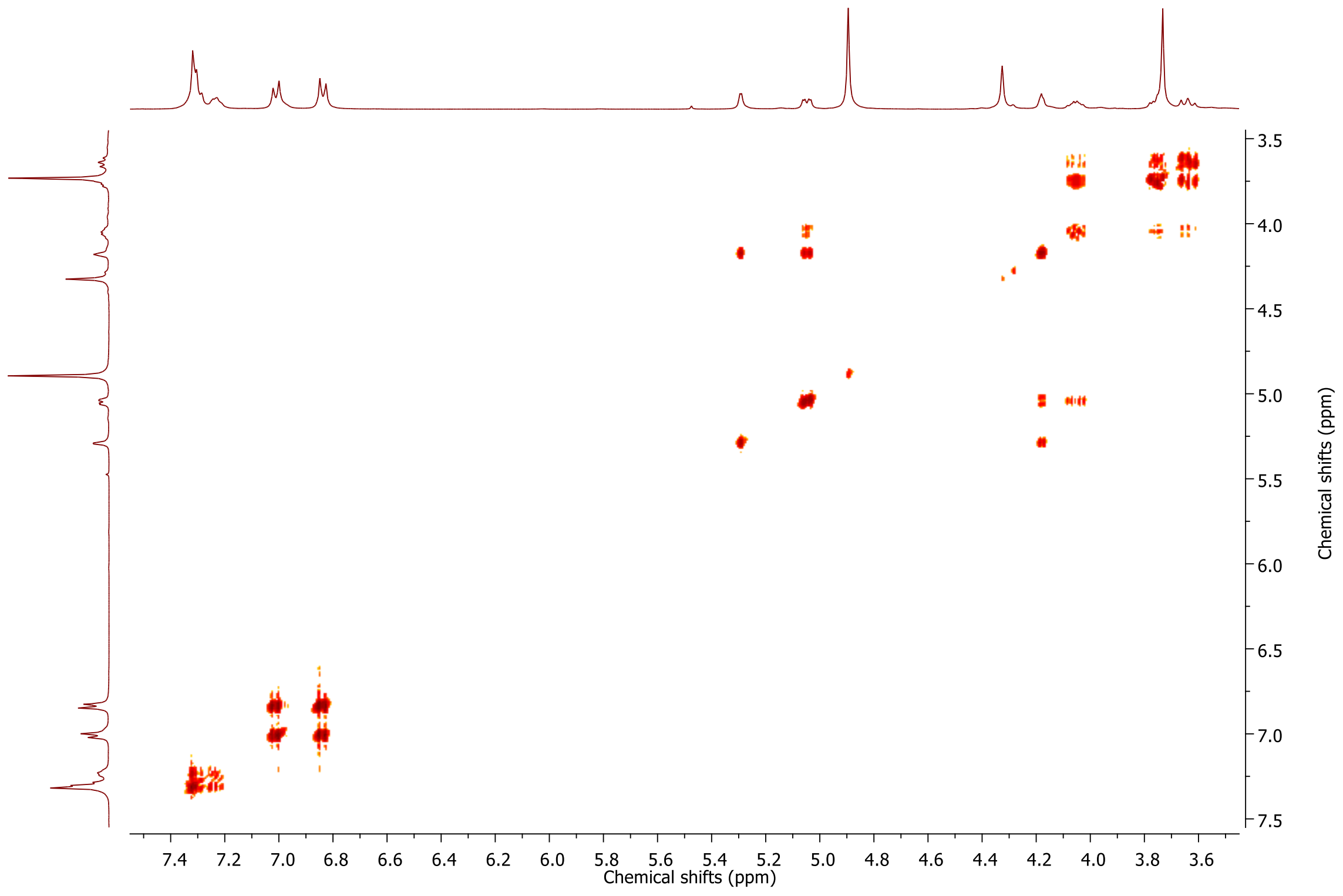


28. ${ }^{1} \mathrm{H},{ }^{13} \mathrm{C}$, and COSY NMR Spectra of compound $9 \mathrm{e}$

a. $\quad{ }^{1} \mathrm{H}$ NMR spectrum $(400 \mathrm{~Hz})$ of 4-methoxyphenyl 3-O-benzylcarbamoyl- $\alpha$-L-rhamnopyranoside $(\mathbf{9 e})$

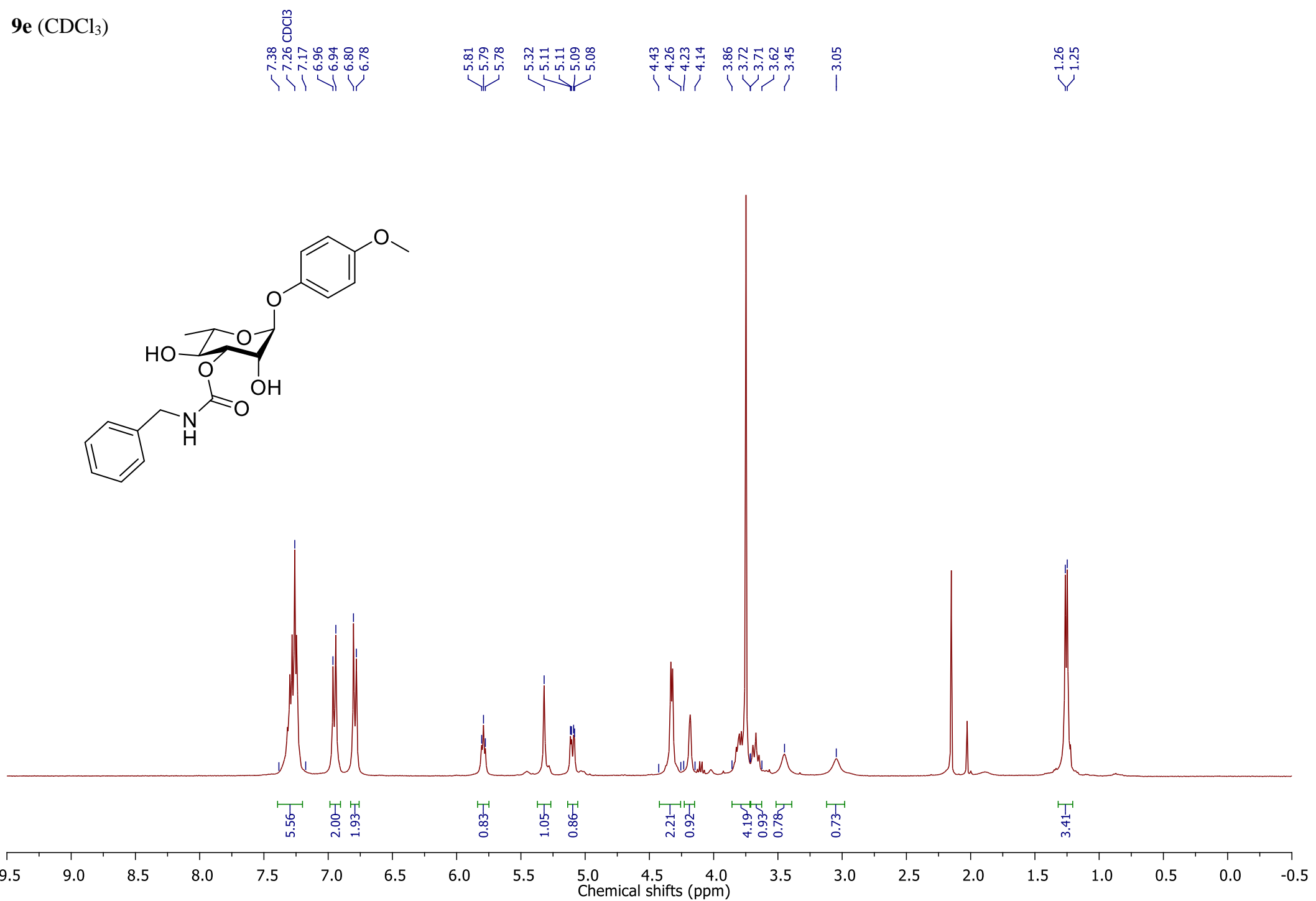


b. ${ }^{13} \mathrm{C}$ NMR spectrum $(101 \mathrm{~Hz})$ of 4-methoxyphenyl 3-O-benzylcarbamoyl- $\alpha$-L-rhamnopyranoside (9e)

9e $\left(\mathrm{CDCl}_{3}\right)$

$$
\text { 药 }
$$
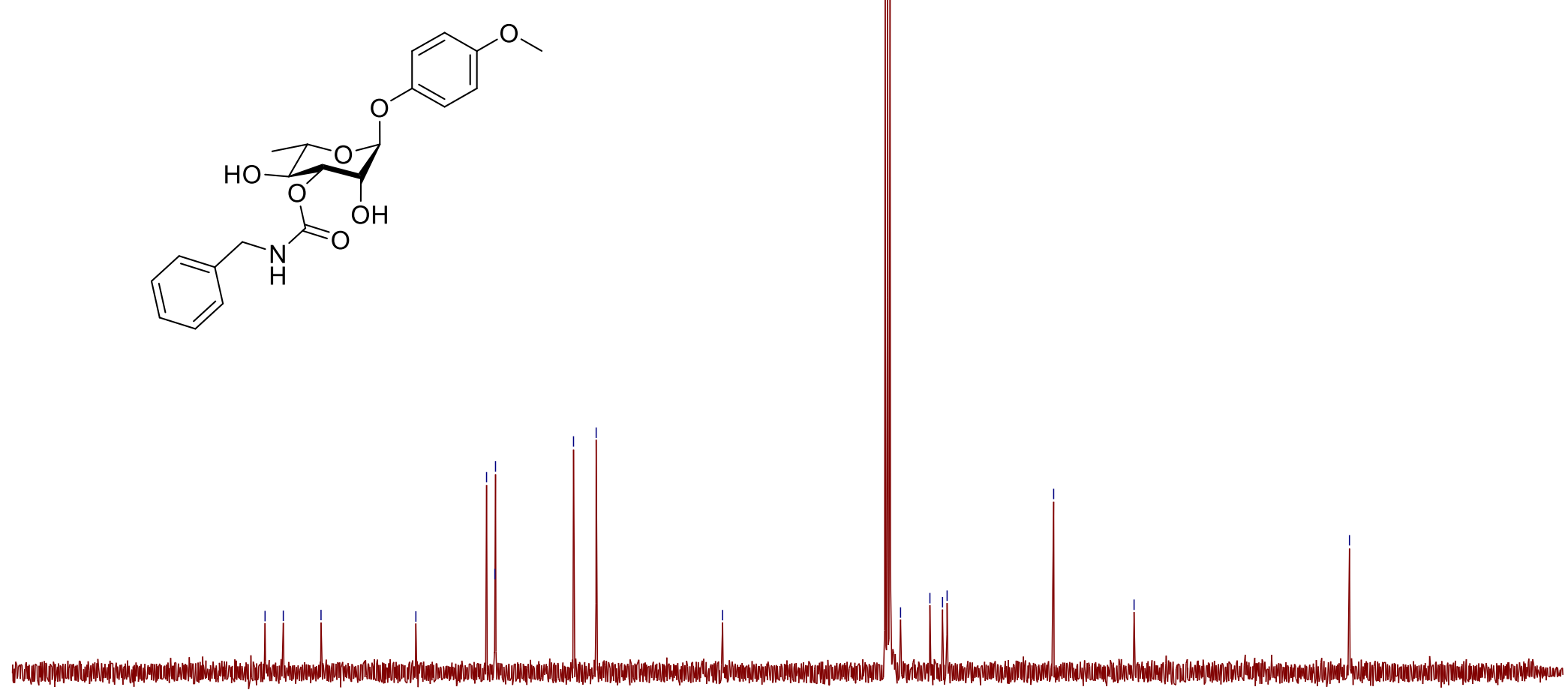
c. COSY NMR spectrum $(400 \mathrm{~Hz})$ of 4-methoxyphenyl 3- $O$-benzylcarbamoyl- $\alpha$-L-rhamnopyranoside $(\mathbf{9 e})$

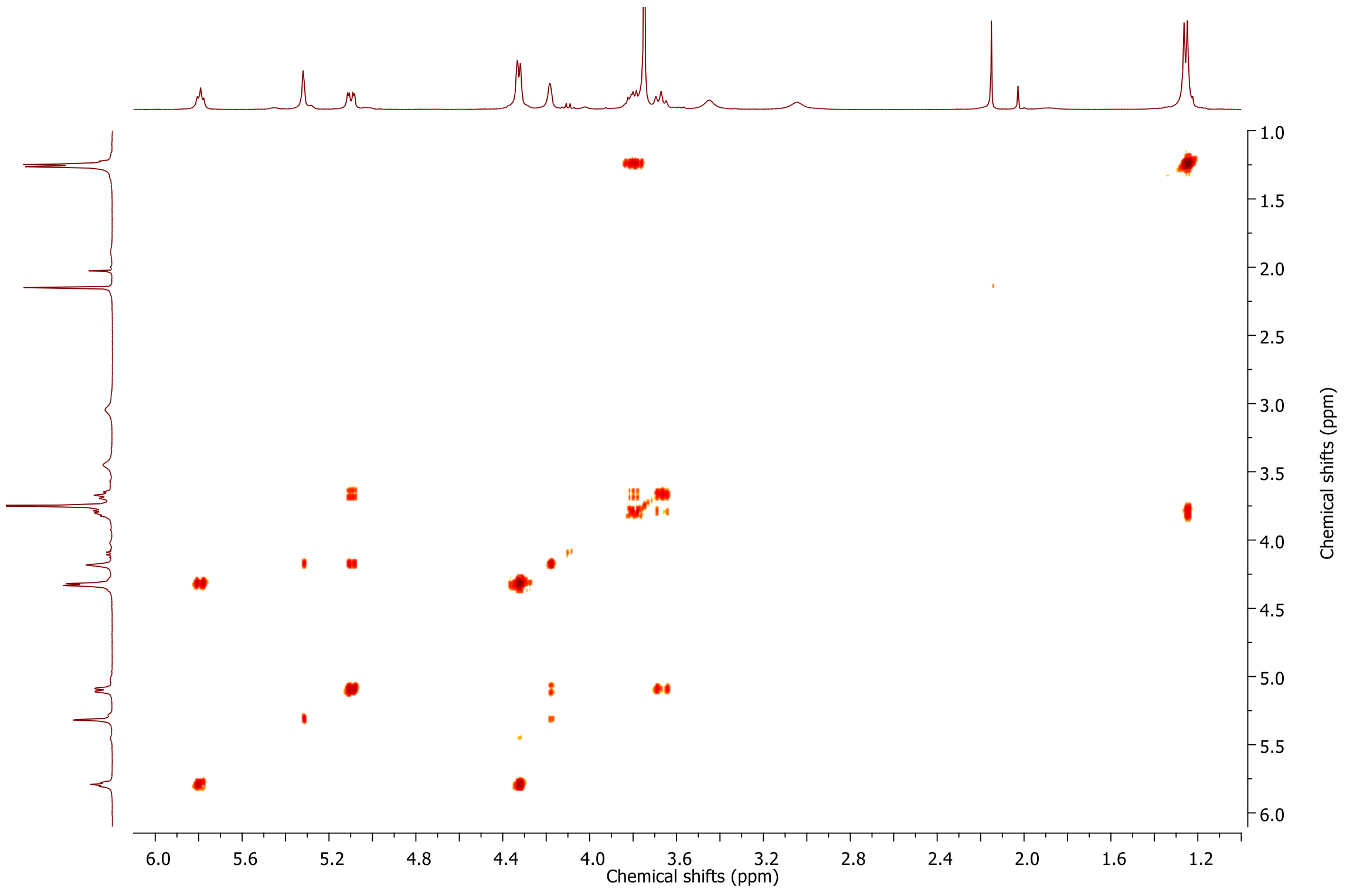

